DOCUMENTS DE TRAVAIL

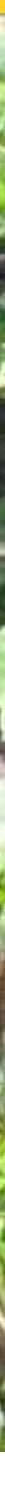

\title{
Projet pilote REDD+ de la Lukénie
}

Flore Hirsh

Jean-Gael Jourget

Laurène Feintrenie

Nicolas Bayol

Richard Eba'a Atyi

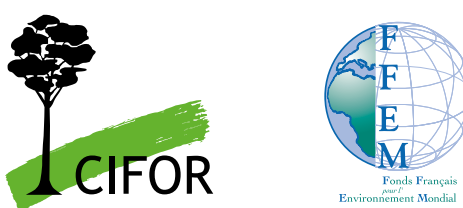





\title{
Projet pilote REDD+ de la Lukénie
}

\author{
Flore Hirsh \\ FRM \\ Jean-Gael Jourget \\ FRM \\ Laurène Feintrenie \\ CIRAD \\ Nicolas Bayol \\ FRM \\ Richard Eba'a Atyi \\ CIFOR
}


Document de Travail 111

(c) 2013 Centre de recherche forestière internationale (CIFOR)

Le contenu de cette publication est soumis à une licence des Creative Commons Commons Attribution NonCommercial-NoDerivs 3.0

Hirsh F, Jourget J-G, Feintrenie L, Bayol, N et Atyi RE. 2013. Projet pilote REDD+ de la Lukénie.

Document de travail 111. Bogor, Indonésie : CIFOR.

Photographie de couverture : FRM

Rouissage et épluchage des tubercules de manioc par les femmes du village d'Essangambala

CIFOR

Jl. CIFOR, Situ Gede

Bogor Barat 16115

Indonésie

$\mathrm{T}+62(251) 8622-622$

$\mathrm{F}+62(251) 8622-100$

Ecifor@cgiar.org

\section{cifor.org}

Tous les points de vue figurant dans cet ouvrage sont ceux des auteurs. Ils ne représentent pas forcément les points de vue du CIFOR, des responsables de la rédaction, des institutions respectives des auteurs, des soutiens financiers ou des relecteurs. 


\section{Table des matières}

Acronymes $\quad$ v

Introduction

1 Présentation du projet pilote 1

1.1 Le projet FORAFAMA 1

1.2 Localisation et présentation des porteurs de projet 1

1.3 Contexte 3

1.4 Objectifs du projet 3

2 Quantification du stock de carbone 7

3 Volet 1 : déforestation évitée 11

3.1 État initial 11

3.2 Élaboration du scénario de référence 11

3.3 Scénario de projet : activités prévues, quantification 14

3.4 Système de suivi 21

3.5 Éléments d'analyse financière 21

4 Volet 2 : exploitation forestière à impact réduit 24

4.1 État initial

4.2 Élaboration du scénario de référence 25

4.3 Scénario de projet : activités prévues, quantification 29

4.4 Système de suivi 31

4.5 Éléments d'analyse financière 32

5 Volet 3 : mise en conservation 34

5.1 Élaboration du scénario de référence 34

5.2 Scénario de projet : activités prévues, quantification 37

5.3 Système de suivi 38

5.4 Éléments d'analyse financière 38

6 Bilan et perspectives 41

6.1 Bilan

6.2 Bénéfices sociaux et environnementaux 42

6.3 Risque et gestion du risque 44

6.4 L'enregistrement du projet 44

7 Conclusion 48 


\section{Liste des cartes, figures et tableaux}

\section{Cartes}

1 Localisation des concessions forestières de la Lukénie 2

2 Projet pilote REDD+ de la Lukénie 5

3 Localisation des layons d'inventaire d'aménagement sur les concessions de la Lukénie $\quad 7$

Répartition des stocks de carbone de la biomasse aérienne sur les concessions
de la Lukénie

5 Images Landsat utilisées pour l'analyse diachronique des concessions
intégrées au Volet 1

6 Dynamique d'anthropisation modélisée sur 20 ans 14

$\begin{array}{ll}7 & \text { Scénario de projet : substitution des pistes principales et secondaires par } \\ \text { des pistes de débardage } & 28\end{array}$

8 Répartition des stocks de carbone contenus dans les arbres du groupe 1
supérieurs à $60 \mathrm{~cm}$ de diamètre

\section{Figures}

1 Modélisation des superficies anthropisées sur les concessions 28/03 et 30/03 13

2 Schéma d'une parcelle agroforestière 16

3 Schéma d'une parcelle agroforestière associée à un enclos et une bergerie $\quad 16$

4 Schéma de la rotation de 6 ans sur 6 ha, avec 1 cycle de manioc suivi $\begin{array}{ll}\text { de } 4 \text { ans de jachère } & 17\end{array}$

5 Modélisation des superficies anthropisées sur les concessions 28/03 et 30/03 18

6 Ventilation du budget total du Volet 1 dans les différentes rubriques 22

7 Vue transversale d'une route forestière 24

8 Protocole de mesure de largeur des routes forestières 26

9 Protocole de terrain : mesure des stocks de carbone forestier 27

10 Ventilation du budget total du Volet 2 dans les différentes rubriques 33

11 Ventilation du budget total du Volet 3 dans les différentes rubriques 39

12 Crédits carbone cumulés produits par les trois volets 42

13 Recettes et dépenses cumulées du Volet $1 \quad 43$

14 Ventilation du budget total du projet dans les différentes rubriques 43

15 Initiatives REDD+ en cours dans le district du Maï Ndombé 46

\section{Tableaux}

1 Émissions de $\mathrm{CO}_{2}$ dans le scénario de référence $\quad 15$

2 Crédits carbone du Volet $1 \quad 19$

3 Emprise spatiale des routes dans le scénario de référence $\quad 27$

4 Superficie affectée par les infrastructures routières selon les différents scénarii $\quad 30$

$5 \quad$ Crédits carbone du Volet 2

6 Incidence de l'exploitation forestière conventionnelle sur les stocks de carbone

7 Crédits carbone du Volet $3 \quad 38$

8 Bilan des trois volets du projet pilote REDD+ de la Lukénie ${ }^{a}$

9 Synthèse des bénéfices environnementaux des trois volets 44

10 Synthèse des bénéfices sociaux et économiques des trois volets 44

11 Risques et gestion du risque 45 


\section{Acronymes}

AFD

AFAT

BL-UP

CIFOR

CIRAD

CCNUCC

DHP

EFIR

FCPF

FFEM

FRM

FSC

GPS

GIEC

IRD

UTCATF

MRV

ONFI

ONG

ONU

PDD

PIN

REDD+

RDC

SIG

SODEFOR

$\mathrm{tCO}_{2} \mathrm{e}$

TEREA

VCS
Agence française de développement

Agriculture, foresterie et autres affectations des terres

Estimation of baseline carbon stock changes and greenhouse gas emissions from unplanned deforestation (estimation de base des variations des stocks de carbone et les gaz à effet de serre dues à la déforestation non planifiée)

Centre de recherche forestière internationale

Centre international de recherche agronomique pour le développement

Convention-cadre des Nations Unies sur les changements climatiques

Diamètre à hauteur de poitrine

Exploitation forestière à impact réduit

Forest Carbon Partnership Facility

Fonds français pour l'environnement mondial

Forêt Ressources Management

Forest Stewardship Council

Global Positioning System

Groupe d'experts intergouvernemental sur l'évolution du climat

Institut de recherche pour le développement

Utilisation des terres, changement d'affectation des terres et foresterie

Mesure, rapport et vérification

Office national des forêts - International

Organisation non gouvernementale

Organisation des Nations Unies

Project design document (document descriptif de projet)

Project idea note (note d'identification de projet)

Réduction des émissions liées à la déforestation et la dégradation des forêts dans les pays en développement, incluant la conservation, la gestion forestière durable et l'augmentation des stocks de carbone forestier

République démocratique du Congo

Système d'information géographique

Société de développement forestier

Tonne de dioxyde de carbone équivalent

Terre Environnement Aménagement

Voluntary Carbon Standard 


\section{Introduction}

Les forêts du bassin du Congo, deuxième plus grande étendue de forêts tropicales du monde, sont au cœur de nouveaux enjeux de services environnementaux, des enjeux mondiaux comme la régulation du climat et la préservation de la biodiversité, ou des enjeux locaux comme la protection de la qualité de l'eau.

La République démocratique du Congo est le pays le plus étendu d'Afrique centrale et $49 \%$ de sa superficie est forestière, représentant 125 millions d'hectares de forêts tropicales, ce qui en fait donc le plus grand pays de forêts tropicales du monde après le Brésil. Mais ces forêts sont exposées à de nombreuses pressions : défrichements pour l'agriculture vivrière, expansion de l'agriculture commerciale à grande échelle, exploitation forestière commerciale non durable, urbanisation, etc. La déforestation et la dégradation forestière sont de plus en plus soutenues ; ainsi, le taux annuel de déforestation nette sur la période 2000-2005 dans les forêts de République démocratique du Congo est de 0,22 \% et est en constante augmentation.

Or, on ne peut que reconnaître le rôle déterminant que les forêts et les arbres jouent dans le développement socio-économique : les forêts contribuent en effet à faire vivre des secteurs clés dans de nombreuses économies africaines, notamment l'agriculture. Plus de $70 \%$ de la population du continent dépend des ressources forestières, principalement de l'énergie (charbon de bois et bois de feu), mais aussi d'autres besoins pour le soutien des moyens de subsistance. Les forêts sont essentielles pour le maintien de la qualité de l'environnement local et pour la fourniture de biens et services publics internationaux.

La République démocratique du Congo et d'autres pays d'Afrique centrale se sont donc engagés à coordonner leurs efforts pour la conservation et la gestion durable de leurs forêts. Afin de lutter contre la déforestation et d'inciter positivement à l'action, le mécanisme de " Réduction des émissions liées à la déforestation et à la dégradation des forêts, incluant la conservation, la gestion forestière durable et l'augmentation des stocks de carbone forestier " (REDD+) est en cours d'élaboration à l'échelle internationale dans le cadre des négociations de la Convention-cadre des Nations Unies sur le changement climatique (CCNUCC). Ces projets ont pour objectif de valoriser les efforts de déforestation évitée ou d'amélioration des stocks de carbone sur le marché du carbone volontaire, qui permet l'échange de crédits carbone (correspondant à des réductions d'émissions, obtenues grâce à la mise en place de projets REDD+).

Le processus REDD avance à petits pas et les pays ont des difficultés à s'engager fortement. Ainsi, le Japon et la Russie se sont même retirés de la deuxième période du protocole de Kyoto (20132020). La France devrait organiser la COP 21 à Paris en 2015, étape importante puisqu'elle devrait déboucher sur un accord juridiquement contraignant (objectif de $+2{ }^{\circ} \mathrm{C}$ maximum, financement, prolongation du protocole de Kyoto, etc.). Mais actuellement, le processus REDD n'existe toujours pas sur le marché réglementé et les projets REDD sur le marché volontaire sont encore rares.

Le projet FORAFAMA a pour objectif de favoriser l'intégration des politiques de gestion durable des forêts dans le mécanisme REDD+. Il se propose de promouvoir l'exploitation durable des ressources ligneuses dans le cadre d'aménagements forestiers compatibles avec la préservation des forêts et de leur stock de carbone, grâce à la mise en œuvre de travaux analytiques, d'actions pilotes de démonstration et à l'échange d'expériences dans le Congo et dans le bassin amazonien.

C'est dans ce cadre que FRM a décidé d'étudier les potentialités d'un projet REDD+ en République démocratique du Congo, dans les concessions forestières de la Lukénie. Ce projet s'articule autour de trois axes :

- La limitation de la déforestation non planifiée ;

- L'exploitation forestière à impact réduit (EFIR) ;

- La mise en conservation volontaire.

La description des différentes activités de ce projet, ainsi que celle des bénéfices et des risques qui en découlent, font l'objet du présent rapport. 


\section{Présentation du projet pilote}

\subsection{Le projet FORAFAMA}

Le projet pilote REDD+ de la Lukénie s'inscrit dans le vaste projet FORAFAMA, qui a pour objectif de promouvoir la gestion durable de deux des plus vastes étendues forestières du monde : celles du bassin du Congo et du bassin amazonien brésilien.

Le projet FORAFAMA est financé par l'Agence française de développement (AFD) et le Fonds français pour l'environnement mondial (FFEM). Le CIFOR, le CIRAD, l'IRD, ONFI, FRM et TEREA ont créé un consortium chargé de mettre en œuvre l'action 1 de la composante 3 de ce projet entre 2010 et 2013, en s'appuyant sur la participation de tous les acteurs concernés par l'aménagement forestier : les gestionnaires des concessions forestières, les responsables des politiques publiques d'aménagement durable des ressources forestières, les responsables nationaux en charge du suivi des initiatives sur les changements climatiques et les représentants de la société civile.

Répondre aux défis du changement climatique fait aujourd'hui partie intégrante de la gestion forestière, notamment à travers les mécanismes REDD+ (réduction des émissions liées à la déforestation et la dégradation des forêts dans les pays en développement, incluant la conservation, la gestion forestière durable et l'augmentation des stocks de carbone forestier). L'aménagement durable des forêts contribue ainsi à réduire la déforestation dans les pays en développement, mais les avantages de cette approche sont encore mal documentés. Le projet FORAFAMA cherche donc à expliciter le rôle de l'aménagement forestier durable dans la lutte contre les émissions de gaz à effet de serre issues de la déforestation et de la dégradation des forêts. Il vise notamment à faire la synthèse des connaissances actuelles sur les liens entre aménagement forestier et changement climatique en Afrique centrale et en Amazonie, à appuyer des projets pilotes REDD+ à l'échelle des concessions forestières, et aider à renforcer les positions des États et des sociétés forestières dans les futurs débats sur la mise en œuvre du processus REDD+.

Plusieurs activités sont prévues pour atteindre ces objectifs, dont notamment une assistance à un

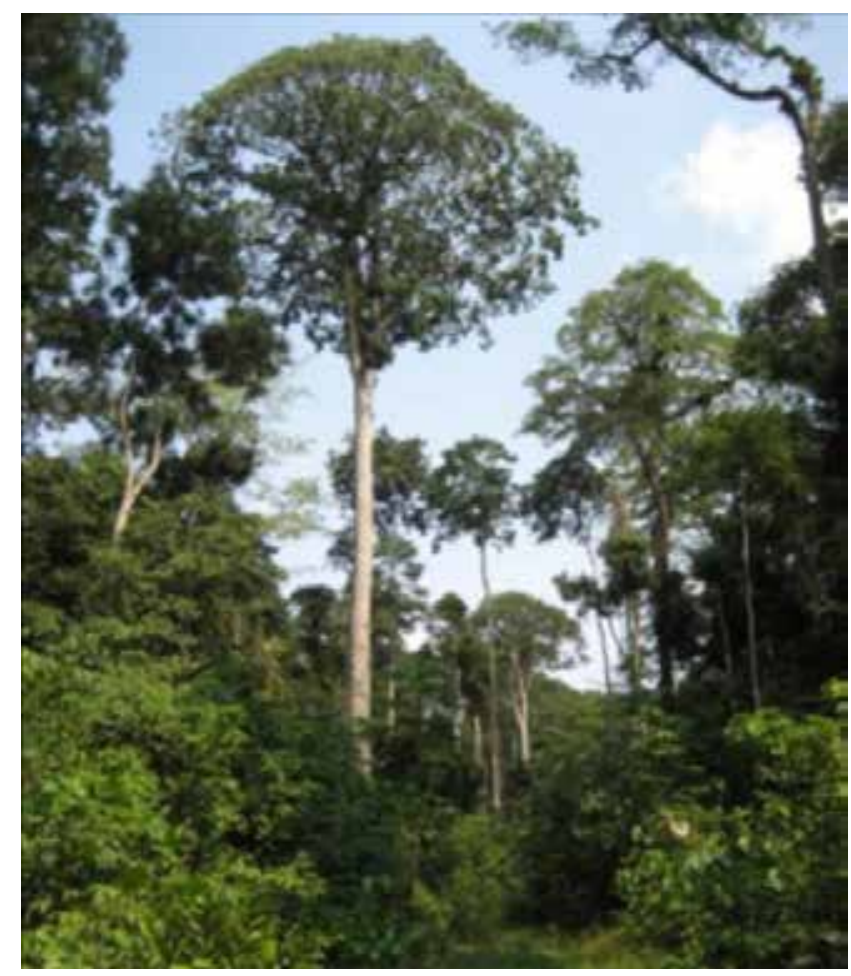

Jeune forêt secondaire Source: FRM 2013

certain nombre d'opérateurs privés souhaitant intégrer la diminution des émissions de carbone dans leur aménagement forestier. Cette assistance a pris la forme d'initiatives pilotes REDD+ dans plusieurs pays du bassin du Congo et au Brésil afin d'aider les concessionnaires à mieux évaluer l'incidence des méthodes d'aménagement forestier. C'est donc dans ce cadre que FRM s'est chargé de mettre en place une initiative pilote REDD+ dans le bassin du Congo et, pour ce faire, a choisi plus précisément les concessions forestières de la Lukénie en République démocratique du Congo (RDC).

\subsection{Localisation et présentation des porteurs de projet}

L'initiative pilote REDD+ de la Lukénie prend place sur le territoire d'Oshwe, dans la province du Bandundu en République démocratique du Congo, au cour de la forêt du bassin du Congo, le deuxième plus grand massif forestier du monde après l'Amazonie (voir Carte 1). Plus précisément, le projet se situe le long de la rivière Lukénie, sur sept titres 


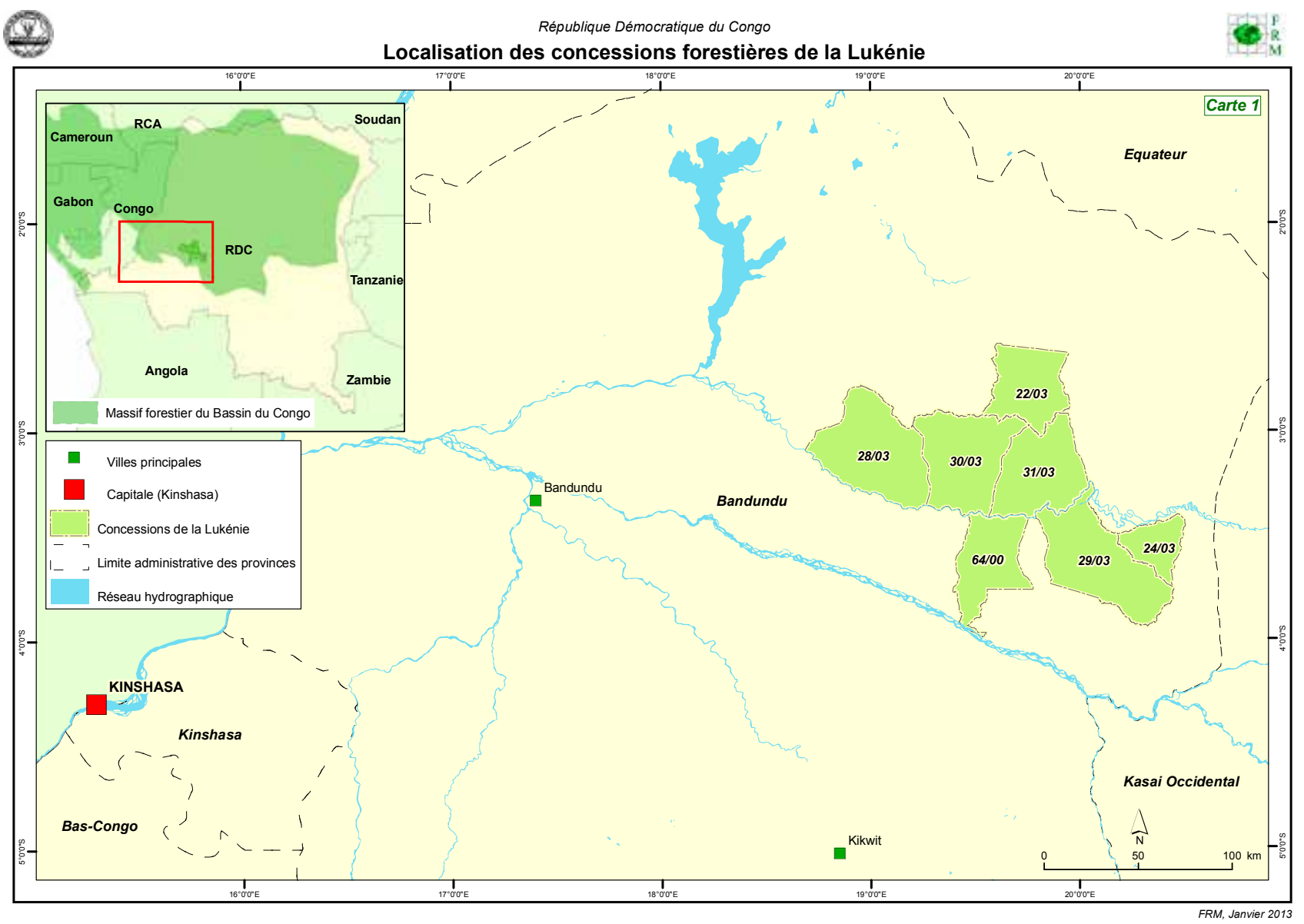

Carte 1 : Localisation des concessions forestières de la Lukénie Source: FRM 2013

forestiers ${ }^{1}$ qui totalisent une superficie de près de 1,3 million d'hectares et qui sont gérés par la Société de développement forestier (SODEFOR), une société forestière privée. L'accès se fait principalement par voie fluviale, sur les $500 \mathrm{~km}$ de voie navigable entre la capitale Kinshasa et les concessions.

La SODEFOR est une société de droit congolais fondée en 1994, présente dans 16 concessions réparties dans trois provinces de RDC. Il s'agit d'un acteur incontournable du secteur forestier national puisque l'entreprise gère 1,8 million d'hectares de surface utile et exploite plus de $50000 \mathrm{~m}^{3} \mathrm{de}$

1 Ces sept titres forestiers sont en cours d'aménagement et ont pour numéro provisoire (en tant que garanties d'approvisionnement) les numéros 22/03, 24/03, 28/03, 29/03, $30 / 03,31 / 03$ et $64 / 00$. Ces garanties sont toutes convertibles en concessions et deux d'entre elles l'ont déjà été récemment : la 28/03, devenue "039/11 Bonkita » et la 31/03, devenue «034/11 Nongenturi ». Étant donné que l'étude de faisabilité du projet pilote REDD+ a été commencée avant la conversion de ces garanties d'approvisionnement en concessions, c'est le numéro provisoire de garantie d'approvisionnement qui sera utilisé dans toute la suite de ce rapport, dans un souci de clarté et d'homogénéité. grumes chaque année. De plus, il s'agit d'une société pionnière, sensibilisée aux problématiques sociales et environnementales : ses concessions forestières sont gérées durablement et sont en voie de certification FSC. La SODEFOR serait en outre la première société forestière privée à accueillir un projet REDD+ en RDC.

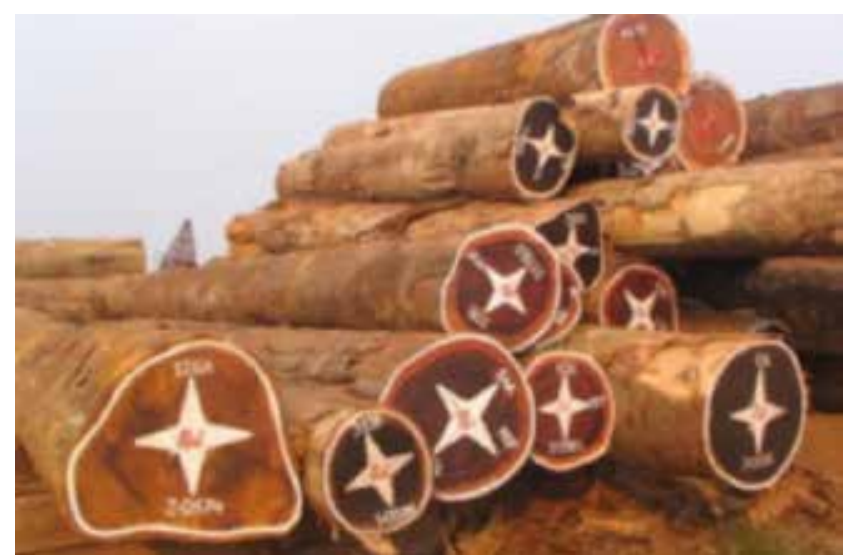

Grumes exploitées par la SODEFOR Source: FRM 2013 
Forêt Ressources Management (FRM) est un bureau d'études français, fondé en 1987, spécialisé dans la foresterie tropicale. Partenaire de la SODEFOR, il élabore les plans d'aménagement des concessions de la société et y a déjà réalisé de nombreuses études. Les concessions de la Lukénie sont particulièrement bien connues : en effet, des inventaires détaillés de la ressource ligneuse (avec un taux de sondage proche de $1 \%$ ) ont déjà été élaborés, ainsi que des études socio-économiques et des inventaires faunistiques.

\subsection{Contexte}

La déforestation, en constante augmentation, est une problématique forte en RDC : évaluée à $0,11 \%$ par an entre 1990 et 2000, elle a atteint 0,22\% par an entre 2000 et 2005 (Ernst et al., 2010).

Les sept concessions de la SODEFOR semblent encore plus touchées par ce phénomène. En effet, situées le long de la rivière Lukénie, les sept concessions actuellement en cours d'aménagement sont soumises à une pression anthropique intense. L'agriculture sur brûlis est une pratique courante. La décroissance rapide de la productivité dans les parcelles gagnées sur la forêt, ainsi que les pratiques agricoles extensives et traditionnelles, obligent la population à cultiver des superficies importantes.

Or, l'accroissement démographique soutenu amène les populations à défricher chaque année de nouvelles surfaces forestières pour répondre aux besoins croissants en produits agricoles. Ce défrichement nécessaire lié à la perte de productivité des sols est d'autant plus important que les sols de ces territoires sont sableux et retiennent peu la matière organique :

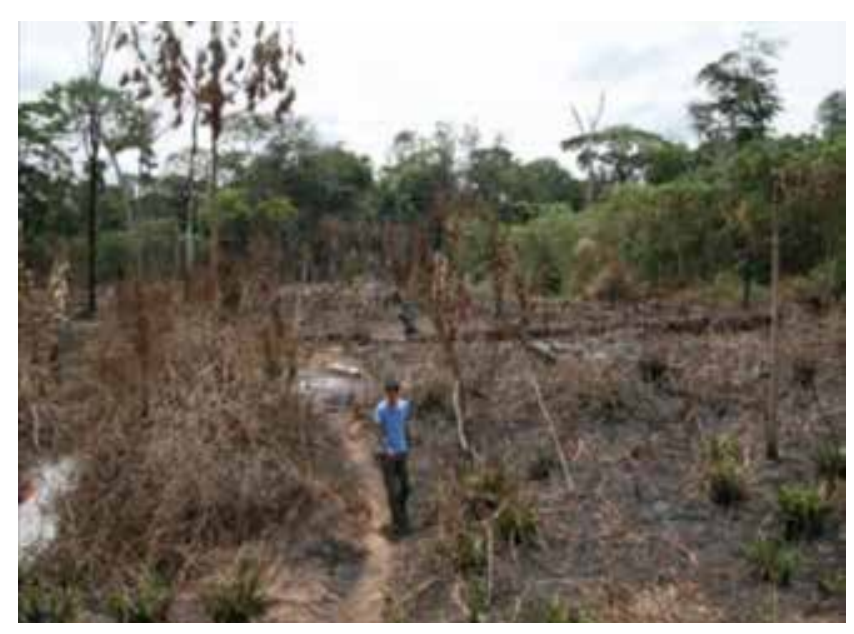

Défrichements agricoles Source: FRM 2013 les sols ne restent fertiles qu'au maximum un ou deux ans après le défrichement.

Les concessions 28/03 et 30/03, par exemple, ont connu un taux de déforestation nette proche de $0,35 \%$ par an entre 2002 et 2007 . Ainsi, près de 12000 ha de forêts ont été défrichés entre 2002 et 2011 pour satisfaire les besoins de l'agriculture vivrière, et si aucun projet n'est mis en place pour freiner ce phénomène, la déforestation cumulée dépasserait 70000 ha en 2031.

\subsection{Objectifs du projet}

$\mathrm{Au}$ vu du contexte décrit ci-dessus, la Lukénie présente diverses possibilités favorables à la mise en place d'un projet REDD+. En effet, il s'agit d'une région forestière, située au cœur du bassin du Congo, le deuxième plus grand massif de forêt tropicale et l'un des plus grands réservoirs de biodiversité du monde. Mais cette forêt est soumise à une pression anthropique forte et connait un taux de déforestation élevé, notamment lié à l'agriculture vivrière constituée de techniques agricoles peu efficaces qui consomment d'importantes superficies forestières. La mise en place d'un projet visant à limiter cette déforestation grandissante prend donc ici tout son sens.

Par ailleurs, la présence de la SODEFOR est tout à fait intéressante, puisque cette société est dans une dynamique d'amélioration de ses pratiques forestières et s'est engagée dans un processus de certification FSC. Elle est donc favorable à la mise en place d'un programme visant à modifier ses pratiques d'exploitation pour maintenir ou améliorer le stock de carbone forestier des concessions.

Enfin, la RDC est le pays africain le plus avancé dans le processus REDD+ : en plus d'avoir ratifié le protocole de Kyoto, le pays adhère au programme ONU-REDD et au FCPF de la Banque mondiale. Un comité national REDD a déjà été établi et la stratégie nationale REDD+ est en cours d'élaboration. Les autorités du pays sont en train de mettre en place un réseau de projets pilotes dans le cadre du développement de la stratégie nationale, et le projet de concession REDD+ s'inscrit tout à fait dans cette démarche. Ce projet pourra donc faire l'objet d'un appui du gouvernement dans le cadre de la mise en ouvre de la stratégie nationale REDD+.

C'est pourquoi il a été décidé d'élaborer un programme REDD+ dans ces concessions, avec pour objectif principal d'étudier la faisabilité de ce 


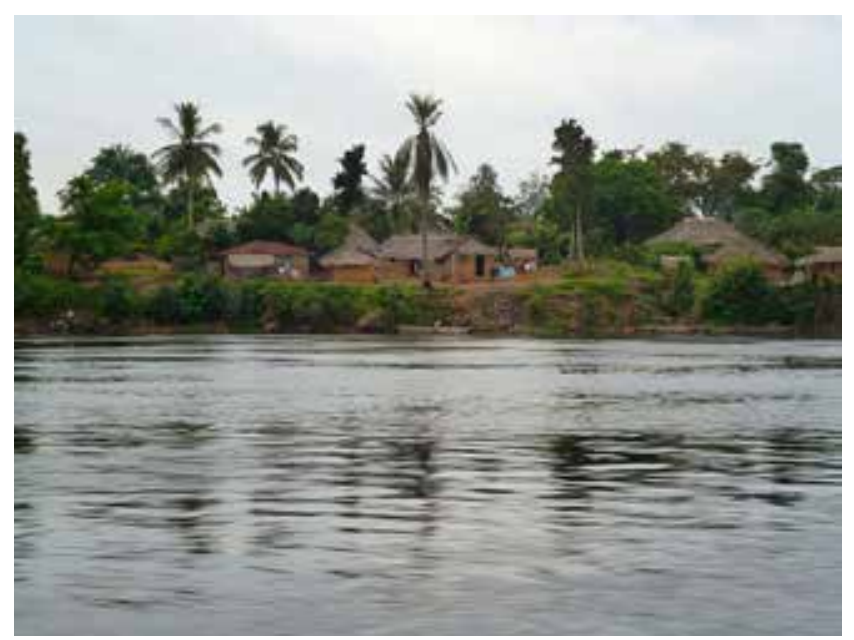

Village sur les rives de la Lukénie Source: FRM 2013

projet REDD+ et sa reproductibilité dans d'autres concessions en République démocratique du Congo ou ailleurs dans le monde. Une note d'identification de projet (PIN) a déjà été réalisée en 2011 afin de présenter le projet et de faire une première évaluation des crédits carbone qui pourraient être délivrés par ce projet. Dans le cadre du présent rapport, les hypothèses ont été affinées et les méthodes de calcul parfois quelque peu modifiées, ce qui a abouti à des estimations légèrement différentes.

Le projet, tel qu'il a été développé, s'articulera autour de trois composantes :

- Volet 1 : déforestation évitée, visant à trouver des solutions pour réduire la dynamique de déforestation liée à l'agriculture vivrière et stabiliser progressivement l'agriculture sur les surfaces déjà défrichées.

- Volet 2 : exploitation forestière à impact réduit (EFIR), visant à réduire les émissions de carbone liées au prélèvement de bois d'œuvre par la SODEFOR.

- Volet 3 : conversion d'une forêt exploitée en réserve intégrale visant à mettre en conservation volontaire (interruption de l'exploitation) une partie des concessions.

Ces trois activités se dérouleront simultanément dans trois zones définies parmi les sept concessions du projet (voir Carte 2).

Le Volet 1 vise à sédentariser l'agriculture pour stabiliser l'avancée du front agricole dans les deux concessions les plus peuplées (28/03 et $30 / 03$, soit une superficie totale de 472790 ha) tout en satisfaisant les besoins des populations locales en produits alimentaires. L'une des solutions de remplacement de l'agriculture sur brûlis qui sera étudiée sera notamment l'agroforesterie. On veillera par ailleurs à limiter les risques de fuite des activités de déforestation hors de la zone du projet.

Une première mission de terrain a été réalisée par Laurène Feintrenie ${ }^{2}$ en mai 2012 dans les concessions de la Lukénie, avec pour objectif principal d'appréhender les systèmes agricoles actuels et leurs contraintes afin de réfléchir aux améliorations possibles. Cette mission s'est fondée sur des enquêtes dans les villages pour établir un diagnostic agraire, ainsi que des diagnostics pédologiques complémentaires.

L'estimation des stocks de carbone qui devraient être préservés grâce au projet a été faite à partir d'un scénario de référence établi en fonction de la dynamique de déforestation observée dans le passé, en se fondant sur la méthodologie VCS VM0007. Les émissions évitées grâce au projet sont estimées à 3,5 millions de tonnes de $\mathrm{CO}_{2}$ en 20 ans.

La description de ce volet est détaillée dans la partie 3.

Le Volet 2 vise à aider le concessionnaire à mettre en œuvre des techniques d'exploitation forestière à impact réduit et à étendre ces pratiques dans cinq concessions (concessions 22/03, 28/03, $30 / 03,31 / 03,64 / 00$, soit une superficie totale de 964114 ha). C'est sur le réseau routier que se

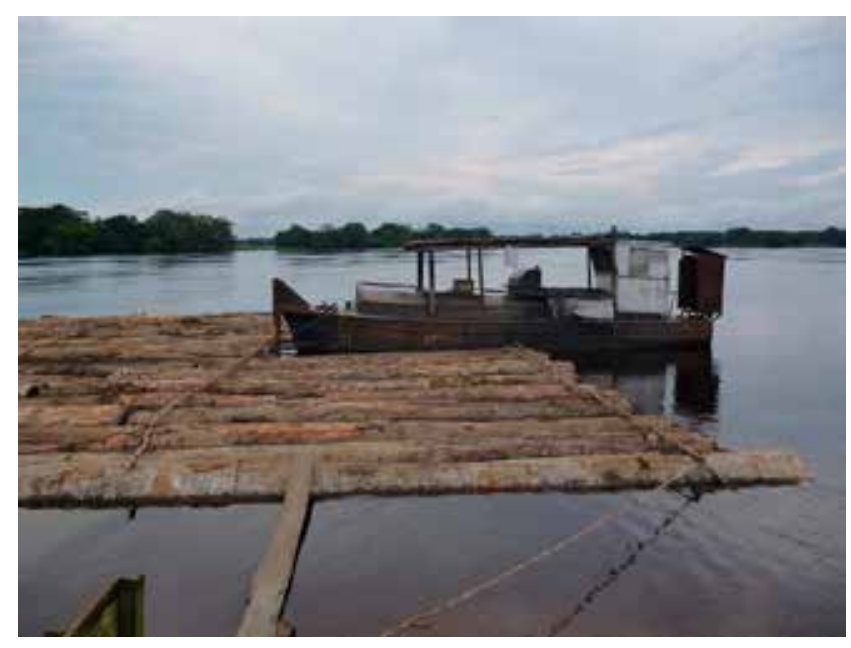

Transport de grumes sur la Lukénie Source: FRM 2013

2 CIRAD, unité de recherche Biens et services des écosystèmes forestiers tropicaux, département Environnements et sociétés. 


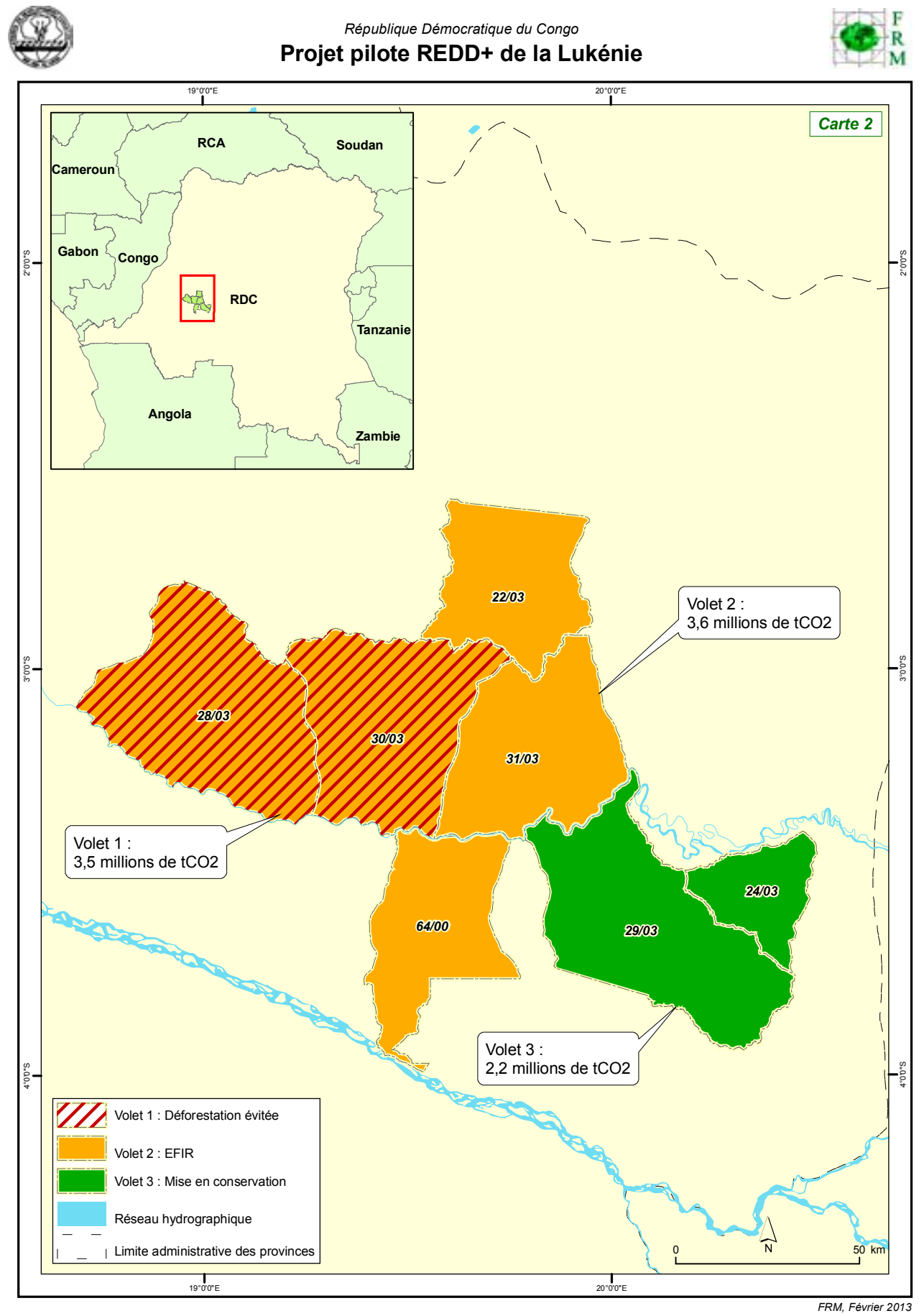

Carte 2 : Projet pilote REDD+ de la Lukénie Source: FRM 2013

concentrera l'étude : l'objectif sera d'optimiser la desserte afin de minimiser la largeur des routes et la longueur du réseau à créer (donc l'incidence directe sur le stock de carbone de la forêt) sans réduire la production de bois d'œuvre. En effet, il s'est avéré que les modalités d'abattage étaient déjà contrôlées et difficilement améliorables. De plus, l'objectif de ce volet est véritablement de réduire l'incidence de l'exploitation sans réduire la production de bois d'œuvre, car cette réduction aurait des conséquences financières importantes pour l'entreprise.

Une première mission de terrain a été réalisée par Jean-Gaël Jourget ${ }^{3}$ et Flore Hirsch ${ }^{4}$ en mai et

3 Forestier aménagiste chez FRM en charge de l'aménagement des concessions SODEFOR.

4 Ingénieur forestier chez FRM, chargée d'études Forêt et Carbone. 
juin 2012 dans les concessions de la Lukénie, afin d'appréhender l'influence du réseau d'exploitation sur la biomasse forestière. Cette mission s'est fondée sur les données de l'inventaire d'aménagement et de mesures sur le terrain à partir d'un protocole établi après concertation avec Lilian Blanc (CIRAD).

À ce jour, il n'existe aucune méthodologie officielle pour quantifier la déforestation évitée grâce aux méthodes EFIR. Il faudra donc développer et faire valider une méthodologie adaptée avant d'enregistrer le projet REDD+. Cette méthodologie est en cours de réalisation ; d'après les estimations, les émissions évitées grâce au projet s'élèveraient ainsi à un total de 3,6 millions de tonnes de $\mathrm{CO}_{2}$ en 20 ans.

La description de ce volet est détaillée dans la partie 4.

Le Volet 3 vise à mettre en conservation volontaire deux des sept concessions du projet : les concessions $24 / 03$ et 29/03, soit une superficie totale de 323287 ha. Ces deux concessions présentent une valeur élevée pour la conservation, puisqu'elles constituent un territoire riche en biodiversité abritant des espèces remarquables comme l'éléphant et le bonobo (rares dans la région) - et bien conservé à ce jour (pas d'infrastructures routières, démographie faible). Le Volet 3 vise donc à mettre un terme à l'exploitation forestière dans ces concessions et à les convertir en concessions de conservation.

L'estimation des stocks de carbone engendrés par cette mise en conservation se fondera sur la méthodologie VCS VM0010 (novembre 2011, version 1.1). Cependant, cette version est en cours de modification depuis septembre 2012 et n'est donc pas valide à ce jour (février 2013). Par défaut, c'est quand même cette version qui a été utilisée pour estimer les émissions évitées grâce au projet ; ainsi, celles-ci s'élèveraient à 2,2 millions de tonnes de $\mathrm{CO}_{2}$ en 20 ans.

La description de ce volet est détaillée dans la partie 5 . 


\section{Quantification du stock de carbone}

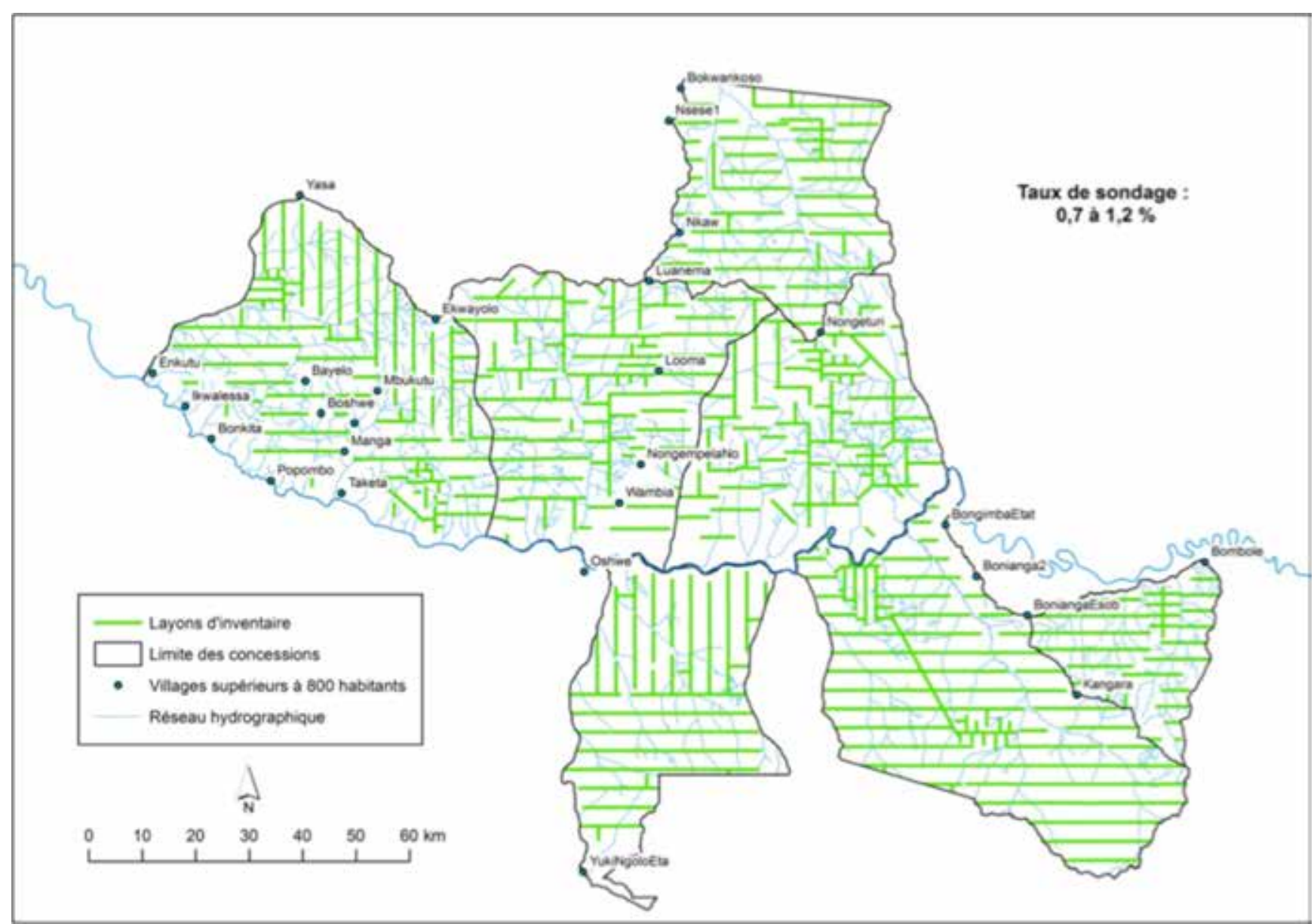

Carte 3 : Localisation des layons d'inventaire d'aménagement sur les concessions de la Lukénie Source: FRM 2013

Les sept concessions de la Lukénie ont bénéficié d'un excellent effort d'inventaire dans le cadre de l'élaboration des plans d'aménagement forestier. Environ $1 \%$ de la surface productive a en effet été inventoriée, ce qui correspond à plus de 16000 placettes de 0,5 ha sur lesquelles a été réalisé un inventaire exhaustif de toutes les tiges supérieures à $10 \mathrm{~cm}$ de diamètre (Carte 3).

L'inventaire d'aménagement permet ainsi d'obtenir une donnée spatialisée très fine sur la ressource en bois (en essences et en diamètre). À partir de ces données, il devient possible de calculer le stock de carbone forestier correspondant à chaque placette.

C'est la méthode allométrique qui a été utilisée pour estimer la biomasse de chaque arbre, en s'appuyant sur l'équation élaborée par Chave et al. $(2005)^{5}$ pour

5 Chave et al. (2005), Tree allometry and improved estimation of carbon stocks and balance in tropical forests. Oecologia, 145, 87-99 les forêts tropicales humides (moist forest stands), une équation allométrique reconnue au niveau international. La biomasse aérienne, qui correspond à la biomasse de la tige, des branches et des feuilles, peut ainsi être estimée grâce à l'équation suivante :

$$
\begin{aligned}
& \mathrm{BA}(\mathrm{kg})=\tilde{\mathrm{n}} \times \exp (-1,499+2,148 \times \ln (D H P)+ \\
& \left.0,207 \times(\ln (D H P))^{2}+0,0281 \times(\ln (D H P))^{3}\right)
\end{aligned}
$$

Où :

- $\quad \mathrm{BA}$ = la biomasse aérienne (en $\mathrm{kg}$ de matière sèche)

- $\tilde{\mathrm{n}}=$ la densité spécifique du bois (gramme de biomasse sèche par $\mathrm{cm}^{3}$ de bois, après séchage à $103^{\circ} \mathrm{C}$ )

- $\mathrm{DHP}=$ le diamètre à hauteur de poitrine $(\mathrm{cm})$

6 Lévapotranspiration étant supérieure aux précipitations pendant 1 à 5 mois par an (entre mai et août), c'est l'équation de type "moist» qui a été retenue, et non l'équation de type "wet" qui convient aux régions dont l'évapotranspiration est inférieure aux précipitations moins d'un mois par an. 
Cette équation a été élaborée pour les forêts tropicales humides (pluviométrie entre 1500 et $3500 \mathrm{~mm}$, saison sèche courte à nulle); son domaine de validité est défini par le DHP, qui doit être compris entre 5 et $156 \mathrm{~cm}$ de diamètre.

Cette équation a été établie grâce à des données de biomasse de 2410 échantillons, mais ceux-ci ont été collectés sur les continents latino-américain et asiatique uniquement : les erreurs liées à l'application de ce modèle en Afrique centrale sont donc inconnues. Cette équation n'en reste pas moins la plus connue et la plus utilisée actuellement. Toutefois, les données d'inventaire recueillies par FRM et SODEFOR pourront faire l'objet de nouveaux traitements si une équation allométrique s'avèrant plus pertinente est disponible.

C'est donc l'équation de Chave qui a été retenue dans notre modèle, car il s'agit de l'équation la plus reconnue et la plus utilisée et, surtout, l'une des rares équations allométriques de forêt tropicale humide à ne pas faire intervenir la hauteur de tige : la hauteur est un paramètre particulièrement difficile à mesurer en forêt tropicale et elle n'a pas été mesurée lors de l'inventaire d'aménagement. Par ailleurs, cette équation fait intervenir la densité spécifique du bois, une variable dont dépend fortement la biomasse, et qui peut quasiment varier du simple au triple selon l'essence : cette variable est donc essentielle pour estimer le stock de carbone du bois. Une base de données recensant la densité du bois de près de 1800 espèces issues de toute la ceinture tropicale a été utilisée.

Une fois la biomasse aérienne obtenue, il s'agit d'obtenir la masse de carbone : il faut alors

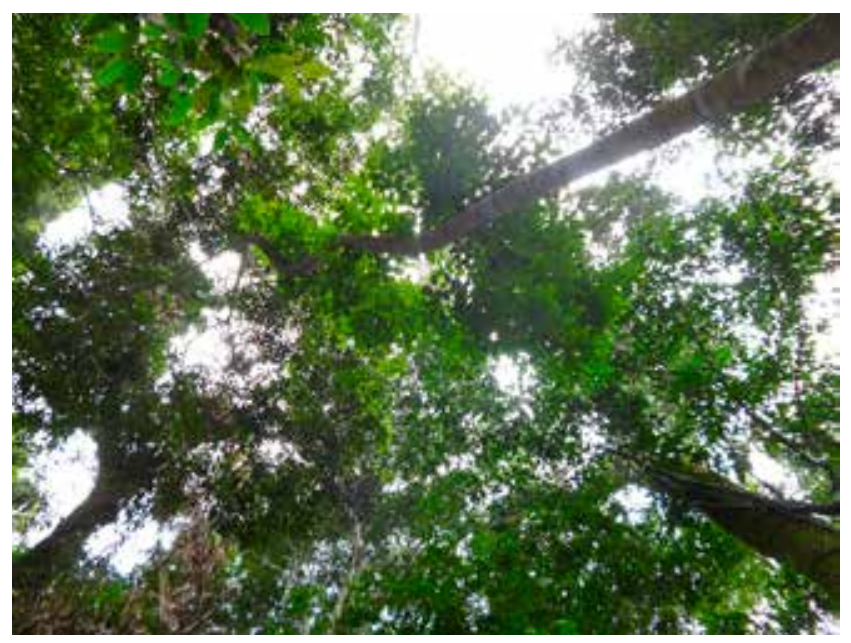

Biomasse aérienne Source: FRM multiplier la biomasse par la fraction de carbone (FC), c'est-à-dire la proportion de carbone dans la biomasse ligneuse sèche, considérée comme égale à 0,5 (valeur par défaut conseillée dans Recommandations en matière de bonnes pratiques pour le secteur de l'utilisation des terres, changements d'affectation des terres et foresterie, Groupe d'experts intergouvernemental sur l'évolution du climat, 2003).

Enfin, la masse équivalente en $\mathrm{CO}_{2}$ est obtenue en multipliant la masse de carbone obtenue précédemment par 44/12 (le carbone constitue en effet $27,3 \%$ de la masse d'une molécule de $\mathrm{CO}_{2}$ ).

Finalement, la masse équivalente en $\mathrm{CO}_{2}$ d'un arbre donné peut être calculée de la manière suivante :

$$
\begin{aligned}
& B A\left(t C O_{2}\right)=(0,5 \times 44 / 12 \div 1000) \times \tilde{\mathrm{n}} \times \\
& \exp (-1,499+2,148 \times \ln (D H P)+0,207 \times \\
& \left.(\ln (D H P))^{2}+0,0281 \times(\ln (D H P))^{3}\right)
\end{aligned}
$$

Où :

- $\mathrm{BA}=$ la biomasse aérienne (en tonnes de $\mathrm{CO}_{2}$ )

- $\quad \tilde{n}$ = la densité spécifique du bois (gramme de biomasse sèche par $\mathrm{cm}^{3}$ de bois, après séchage à $103^{\circ} \mathrm{C}$ )

- $\mathrm{DHP}=$ le diamètre à hauteur de poitrine $(\mathrm{cm})$

Un logiciel a été mis au point par FRM, Forest Carbon Print-Stock v.1, afin de calculer la biomasse en carbone de chaque arbre, par essence et par classe de diamètre, en faisant intervenir une base de données indiquant la densité du bois de plus de 1800 espèces issues de la ceinture tropicales, puis de compiler ces données par placette. Grâce aux coordonnées géographiques de ces placettes, il est alors possible d'exporter au format shapefile les données de stock de carbone par placette, puis d'interpoler ces données sur ArcGIS afin d'obtenir une carte de répartition des stocks de carbone (voir Carte 4).

Si une autre équation allométrique, mieux adaptée au contexte local et notamment africain, est mise à disposition, les calculs de stocks de carbone pourront être renouvelés à partir des données d'inventaire. De même, il pourra être envisagé à l'avenir d'améliorer l'évaluation en complétant les données de base disponibles par des mesures de hauteur sur un souséchantillon.

On remarque dès lors une grande hétérogénéité dans la répartition des stocks de carbone : ils sont plus faibles à l'orée des savanes marécageuses (le long de 


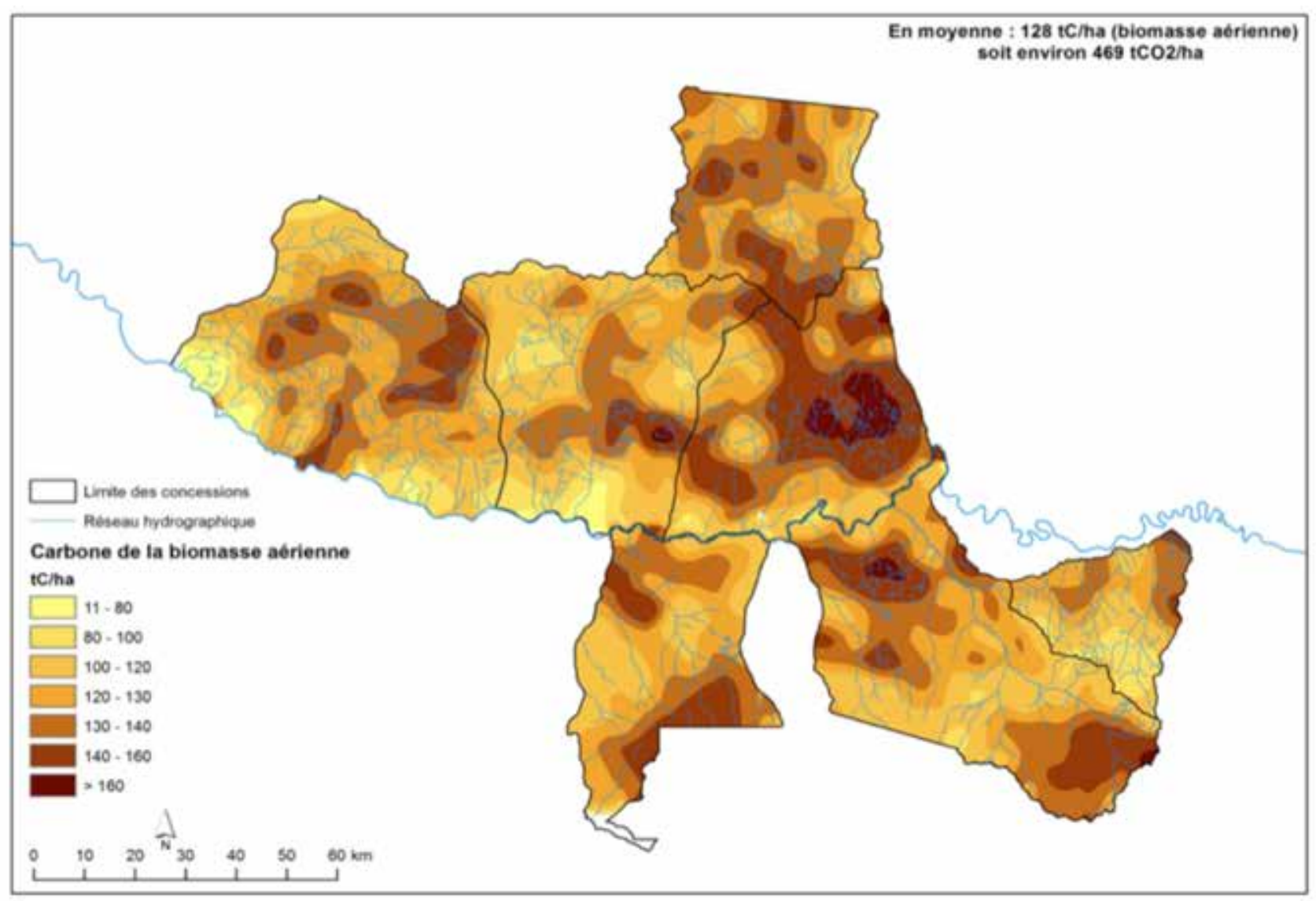

Carte 4 : Répartition des stocks de carbone de la biomasse aérienne sur les concessions de la Lukénie Source: FRM 2013

la Lukénie) et augmentent avec la densité de la forêt ; ils deviennent particulièrement importants dans certaines zones forestières lorsqu'elles sont composées de bois lourds.

Sur la superficie utile (inventoriée lors de l'inventaire d'aménagement) de l'ensemble des sept concessions concernées par le projet, le stock de carbone moyen est de $128 \mathrm{tC} / \mathrm{ha}$, soit $469 \mathrm{tCO}_{2} \mathrm{e} / \mathrm{ha}$. Plus précisément, les deux concessions $28 / 03$ et 30/03 (Volet 1) présentent un stock moyen de $449 \mathrm{tCO}_{2} \mathrm{e} /$ ha, les cinq concessions du Volet 2 un stock moyen de $471 \mathrm{tCO}_{2} /$ ha et les concessions 24/03 et 29/03 $\mathrm{du}$ Volet 3 un stock moyen de $464 \mathrm{tCO}_{2} /$ ha. Pour la suite des calculs, ce sont ces valeurs moyennes qui seront utilisées.

Une mise en garde s'impose en ce qui concerne la précision des résultats, qui est difficile à évaluer. Les incertitudes interviennent à différents niveaux du calcul :

- Erreur liée à l'équation allométrique, d'autant plus que celle-ci n'est pas adaptée au contexte africain : en effet, aucun des modèles existants, même les plus récents, n'utilisent de données provenant des forêts tropicales d'Afrique ${ }^{7}$;

- Erreur attribuable au choix du modèle allométrique pour dériver la biomasse à partir d'autres paramètres ;

- Incertitude liée à l'échantillonnage.

Les inventaires d'aménagement donnent une précision de résultats excellente sur les effectifs (erreur relative inférieure à $1 \%$ sur l'ensemble du massif de la Lukénie), grâce aux méthodes d'échantillonnage et à un protocole de terrain rigoureusement établi (inventaire exhaustif de toutes les tiges jusquà $10 \mathrm{~cm}$ de diamètre, sur des placettes relativement grandes ( $0,5 \mathrm{ha})$, couvrant au total $1 \%$ de la superficie utile). L'essentiel de l'incertitude est donc lié à l'équation allométrique et à la conversion du diamètre de l'arbre en biomasse de carbone. Cette incertitude

7 Nasi et al. (2008), Un aperçu des stocks de carbone et leurs variations dans les forêts du bassin du Congo, État des forêts, chapitre 12. 
ne peut être évaluée actuellement ; des mesures de biomasse avec une méthode destructive seraient en effet nécessaires pour comparer les données de terrain avec les données modélisées grâce à l'équation allométrique, et ainsi évaluer le pourcentage d'erreur. Cependant, on peut supposer que cette erreur est relativement élevée; l'évaluation des stocks de carbone pourra être reprise lorsque des équations allométriques plus adaptées auront été développées en Afrique centrale.
Les pressions anthropiques qui pèsent sur les forêts de la Lukénie ont été décrites dans la première partie de ce rapport. Des activités visant à limiter la déforestation et la dégradation des forêts, en s'adaptant aux problématiques et aux enjeux précédemment mis en évidence, sont proposées et analysées dans les parties suivantes. L'incidence de ces activités sur le stock de carbone forestier sera estimée en s'appuyant sur la méthodologie et les calculs expliqués ci-dessus. 


\section{Volet 1 : déforestation évitée}

\section{1 État initial}

Le climat de la région est caractérisé par une pluviométrie importante, de l'ordre de $1800 \mathrm{~mm}$ par an, répartie en une saison pluvieuse de septembre à mai, et une saison sèche de juin à août.

Les sols sont sableux, à faible teneur en argile (moins de $20 \%$ ) et pauvres en matière organique (Menga Munkolo, 2011). Un phénomène de lixiviation des argiles a été mis en évidence : la migration verticale des argiles entraîne localement la formation d'une cuirasse latéritique, imperméable, au-dessus de la roche mère. Suite à cela, les éléments organiques ne pouvant plus s'infiltrer sont lessivés latéralement, dans le sens de la pente principale, jusqu'à la rivière. Les horizons superficiels deviennent alors sableux, sensibles à l'érosion, au lessivage et au tassement. Après défrichement, les sols sont donc particulièrement fragiles et présentent une perte brutale de fertilité, devenant ainsi rapidement impropres à la culture, ce qui explique les systèmes traditionnels d'agriculture sur brûlis et la dynamique d'agriculture de front pionnier avec une déforestation sans cesse grandissante.

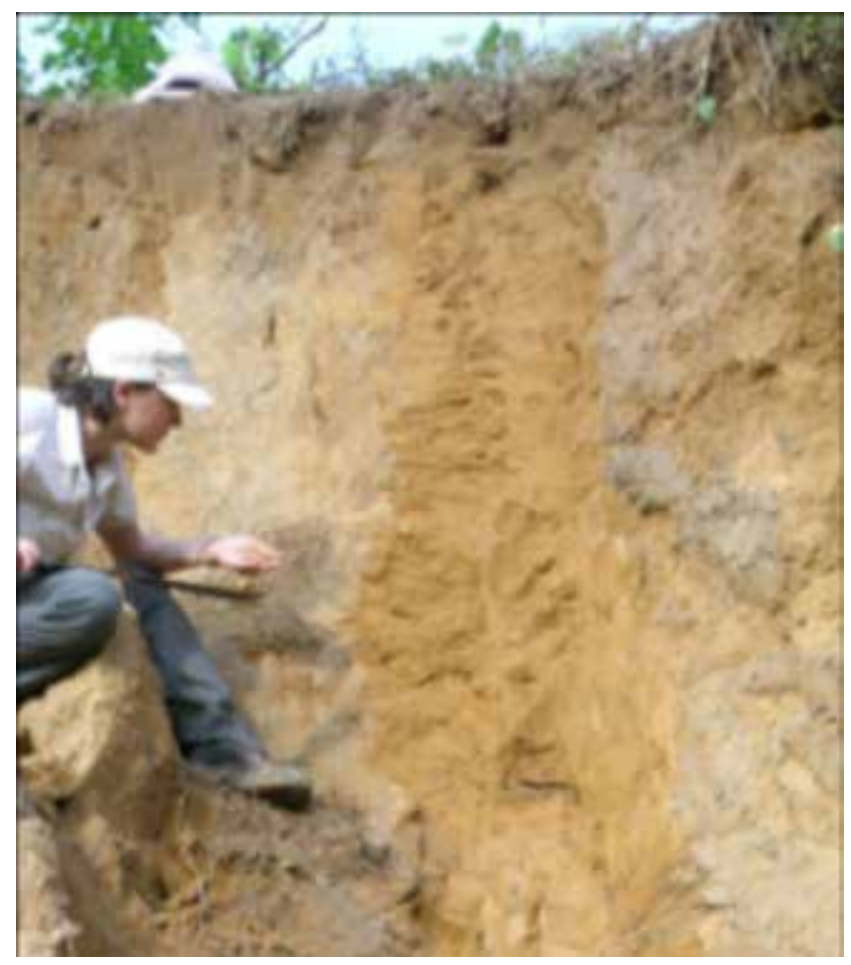

Profil pédologique dans le village de Nténo Source: FRM 2013
L'agriculture est tournée vers l'autosuffisance alimentaire. Le manioc est la culture principale, en mélange avec du maïs, des bananiers, des ananas, des papayers, quelques palmiers à huile, des courges (Feintrenie, 2012). Le maïs est la deuxième culture la plus abondante dans les champs de manioc. Les autres cultures sont présentes en moindre quantité. Le riz est parfois cultivé pour la vente à Kinshasa, séparément des autres cultures. Quelques légumes et condiments sont également cultivés dans des jardins proches des maisons : piment, oseille, haricots, épinards, tomates, canne à sucre.

La mise en culture commence par le défrichement d'une parcelle de forêt ou d'une jachère arborée. Les hommes disent revenir sur d'anciens champs de manioc après 5 ou 7 ans de jachère ; à l'inverse, les femmes disent en majorité ne jamais revenir sur une ancienne parcelle, mais au contraire cultiver chaque année un nouveau champ ouvert sur la forêt. Cependant, les observations sur le terrain et l'analyse des images satellite semblent démontrer que les jachères sont remises en culture puisque les villages ne sont pas encerclés de recrû forestier et de parcelles abandonnées. Une enquête plus approfondie sera donc nécessaire pour vérifier ce qu'il en est et estimer le temps réel moyen de mise en jachère avant la potentielle remise en culture.

Aucune fertilisation n'est effectuée, ni chimique ni organique. La mosaïque du manioc reste la principale maladie touchant la production.

L'élevage est quant à lui peu développé. Il s'agit principalement d'élevage ovin, en divagation, et ne bénéficiant d'aucune prophylaxie.

\section{2 Élaboration du scénario de référence}

Afin d'estimer l'évitement d'émissions lié au projet, il est nécessaire d'établir un scénario de référence, c'est-à-dire de déterminer ce qu'il se passerait si aucun projet de réduction d'émissions n'était mis en place. Il s'agit donc de se fonder sur une analyse des données historiques de déforestation pour modéliser un scénario probable de dynamique future de déforestation. Ce scénario de référence a été établi à l'aide du module BL-UP de la méthodologie 


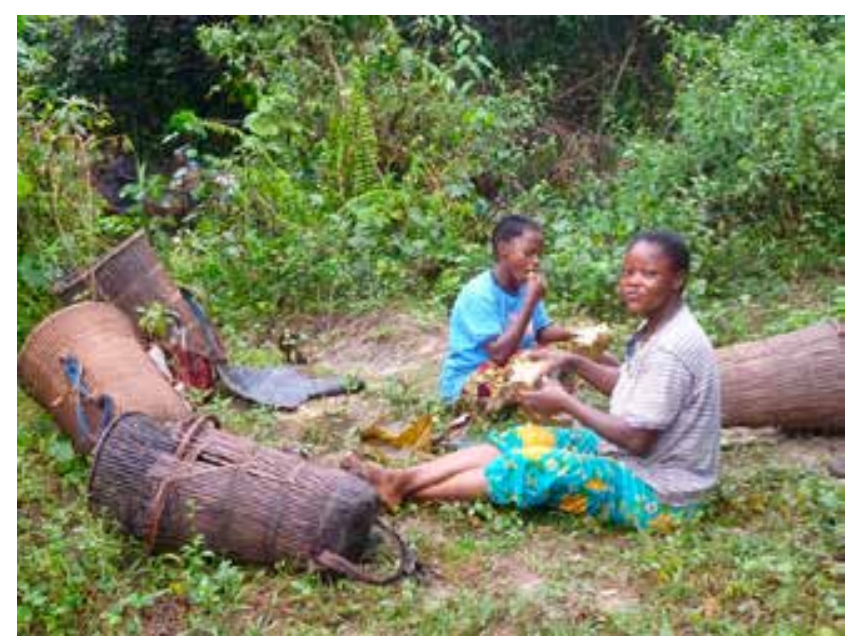

Moment de pause pendant la préparation des tubercules de manioc

Source: FRM 2013

VCS VM0007 : Estimation of baseline carbon stock changes and greenhouse gas emissions from unplanned deforestation (novembre 2012, v 3.1).

Le taux de déforestation annuel lié à une déforestation non planifiée (cas de l'agriculture vivrière sur brûlis) peut ainsi être estimé à partir d'une analyse diachronique d'images satellite dans une zone de référence, cette zone devant être géographiquement proche de la zone de projet et présenter des facteurs de déforestation similaires. En effet, cette zone devra continuer à servir de référence (ou de "zone témoin ») une fois le projet commencé, lors de la phase de vérification pour délivrer les crédits carbone. Ici, dans un premier temps et pour une première approximation, la zone de référence utilisée correspond exactement à la zone du projet. Mais lorsque le projet entrera en phase d'enregistrement, l'étude devra être approfondie et porter sur une zone géographiquement différente de la zone de projet.

D'après la méthodologie VM0007, les images satellite utilisées pour l'analyse diachronique doivent présenter une résolution de $30 \mathrm{~m}$ x $30 \mathrm{~m}$ (ou une résolution plus fine), ce qui correspond à des clichés Landsat. L'analyse doit porter sur trois images au moins, couvrant une période de 12 ans maximum, dont une image datant de moins de deux ans avant la date de début du projet. Les six clichés qui ont ainsi

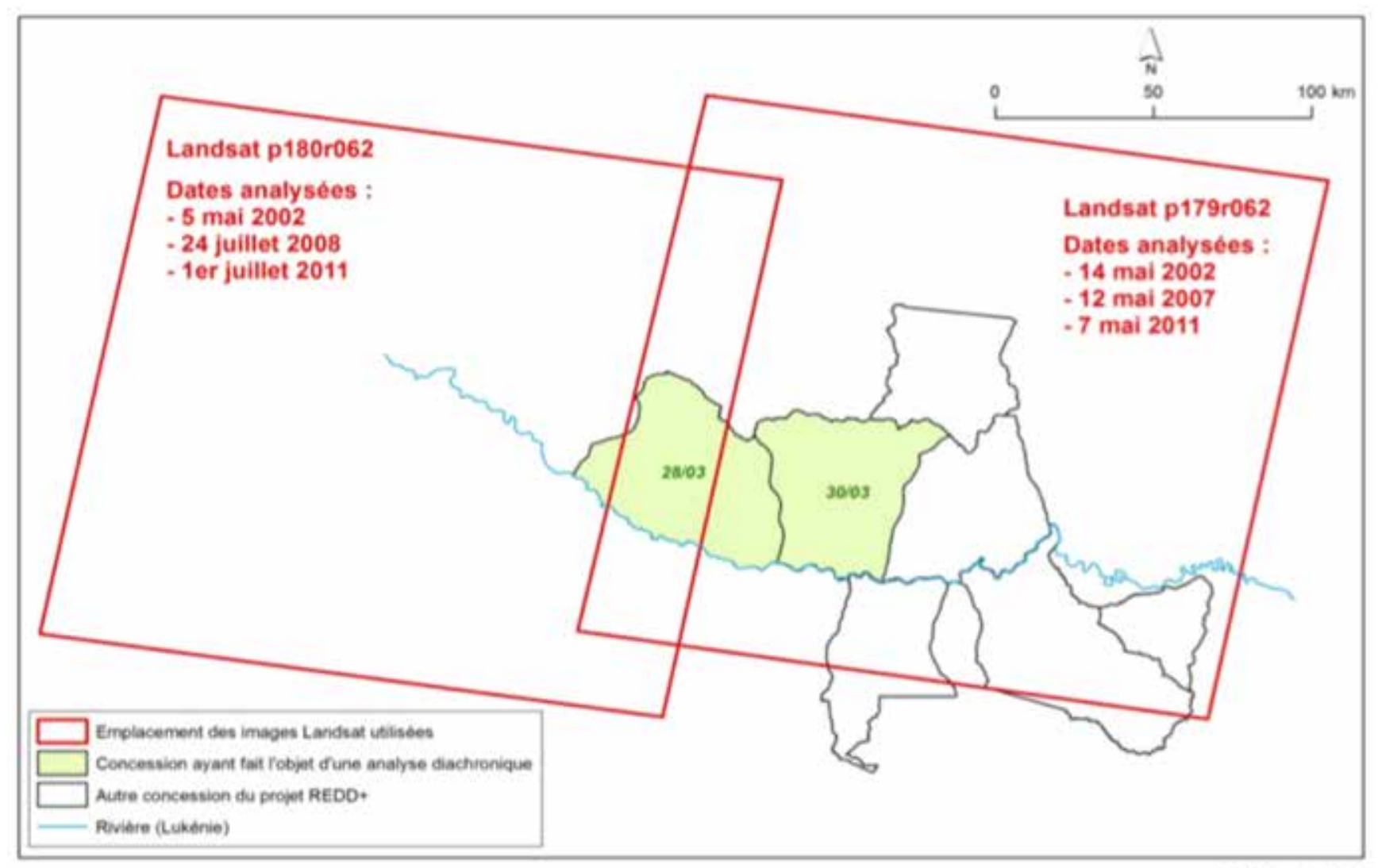

FRM Janvier 2013

Carte 5 : Images Landsat utilisées pour l'analyse diachronique des concessions intégrées au Volet 1 Source: FRM 2013 
été utilisés pour l'analyse diachronique sont listés sur la carte ci-dessous (Carte 5).

À partir de ces images satellite, les superficies anthropisées (cultures, jachères, habitations) ont été numérisées pour chacune des trois années étudiées : on obtient alors la dynamique de défrichement, qui atteint presque 1300 ha défrichés par an entre 2002 et 2011 sur les concessions 28/03 et 30/03.

Il est également possible de calculer le taux annuel de déforestation nette par la formule de la FAO (1995) :

$$
\mathrm{q}=(\mathrm{A} 2 / \mathrm{A} 1)^{(1 /(2-1-1))}-1
$$

Où :

- $\mathrm{q}=$ taux annuel de déforestation (en \% par an)

- $\mathrm{A}=$ superficie forestière au temps $\mathrm{t}$

- $\quad \mathrm{t} 2-\mathrm{t} 1=$ intervalle de temps entre les dates d'observation des défrichements (en années)

Entre 2002 et 2011, le taux annuel de déforestation nette observé est ainsi de $\mathrm{q}=0,30 \%$.

Notons que la formule de Puyravaud (2002)

$$
\mathrm{r}=(1 /(\mathrm{t} 2-\mathrm{t} 1)) \times \ln (\mathrm{A} 2 / \mathrm{A} 1)
$$

donne des résultats similaires $(\mathrm{r}=0,30 \%$ pour la période 2002-2011).

Enfin, il est également possible, par une simple extrapolation linéaire, d'estimer les superficies qui risquent d'être déboisées pendant les années à venir en l'absence de projet REDD+ (voir Figure 1). L'extrapolation linéaire est en effet requise par le module BL-UP lorsque seulement trois images ont été utilisées lors de l'analyse diachronique. Cependant, il s'agit d'une hypothèse prudente : avec la croissance démographique notable de la région, il est fort probable en effet que la dynamique de déforestation soit plus importante que celle estimée ici. Ce sera toutefois le scénario de référence retenu pour la suite des calculs, par mesure de conservation.

La superficie occupée par les villages et les activités agricoles, qui est passée de 32662 ha en 2002 à 44312 ha en 2011, devrait ainsi atteindre 72791 ha en 2033.

Cette dynamique de déforestation peut également être modélisée spatialement, en supposant une expansion par noyau autour des zones anthropisées actuelles et des réseaux de circulation (routes, rivière), et en s'appuyant sur l'observation des dynamiques passées grâce à l'analyse diachronique. Une carte représentant cette dynamique passée et future a ainsi été dressée à titre indicatif (voir Carte 6).

La modélisation représentée sur la Figure 1. estime donc le taux de déforestation à 1294 ha/an pendant toute la durée du projet. À cette superficie de forêt correspond un stock de carbone : en effet, d'après l'inventaire d'aménagement, le stock moyen de carbone forestier dans les concessions 28/03 et 30/03 est égal à $123 \mathrm{tC} / \mathrm{ha}$ (voir Carte 4) soit $449 \mathrm{tCO}_{2} \mathrm{e} /$ ha. Ainsi, cela nous permet de calculer les émissions de $\mathrm{CO}_{2}$ liés à la déforestation telle qu'elle a été

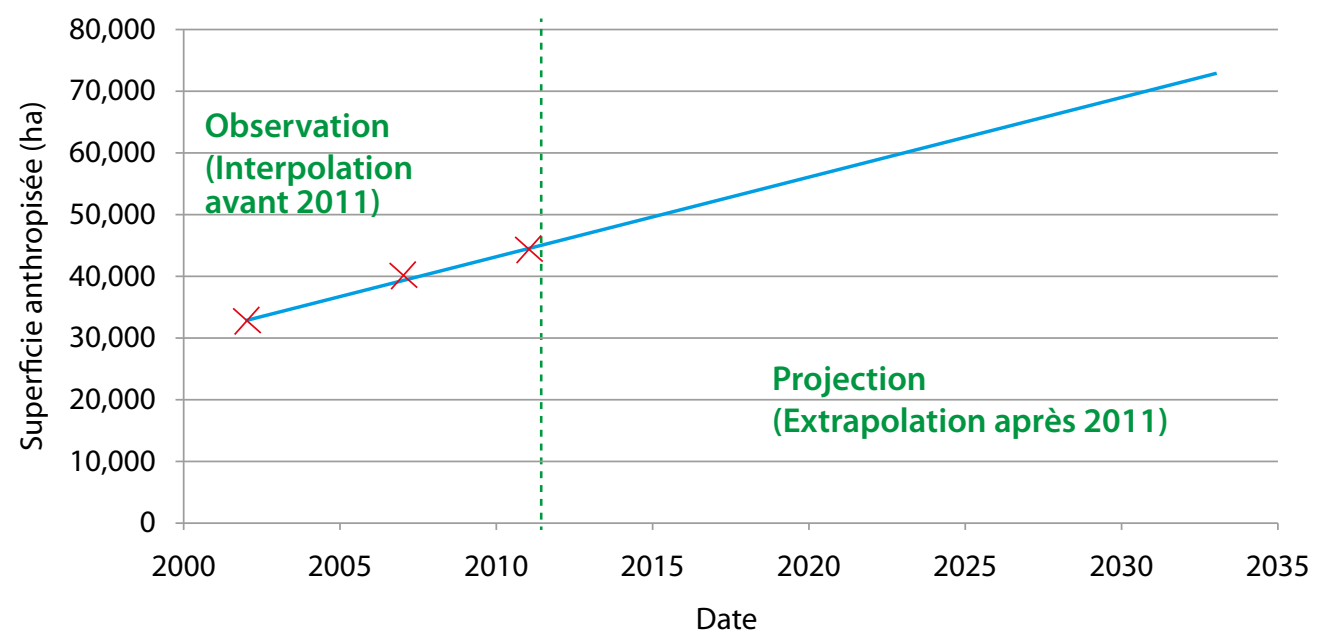

X Surface anthropisée réelle mesurée — Modélisation de la superficie des zones anthropisées

Figure 1 : Modélisation des superficies anthropisées sur les concessions 28/03 et 30/03 (scénario de référence) 


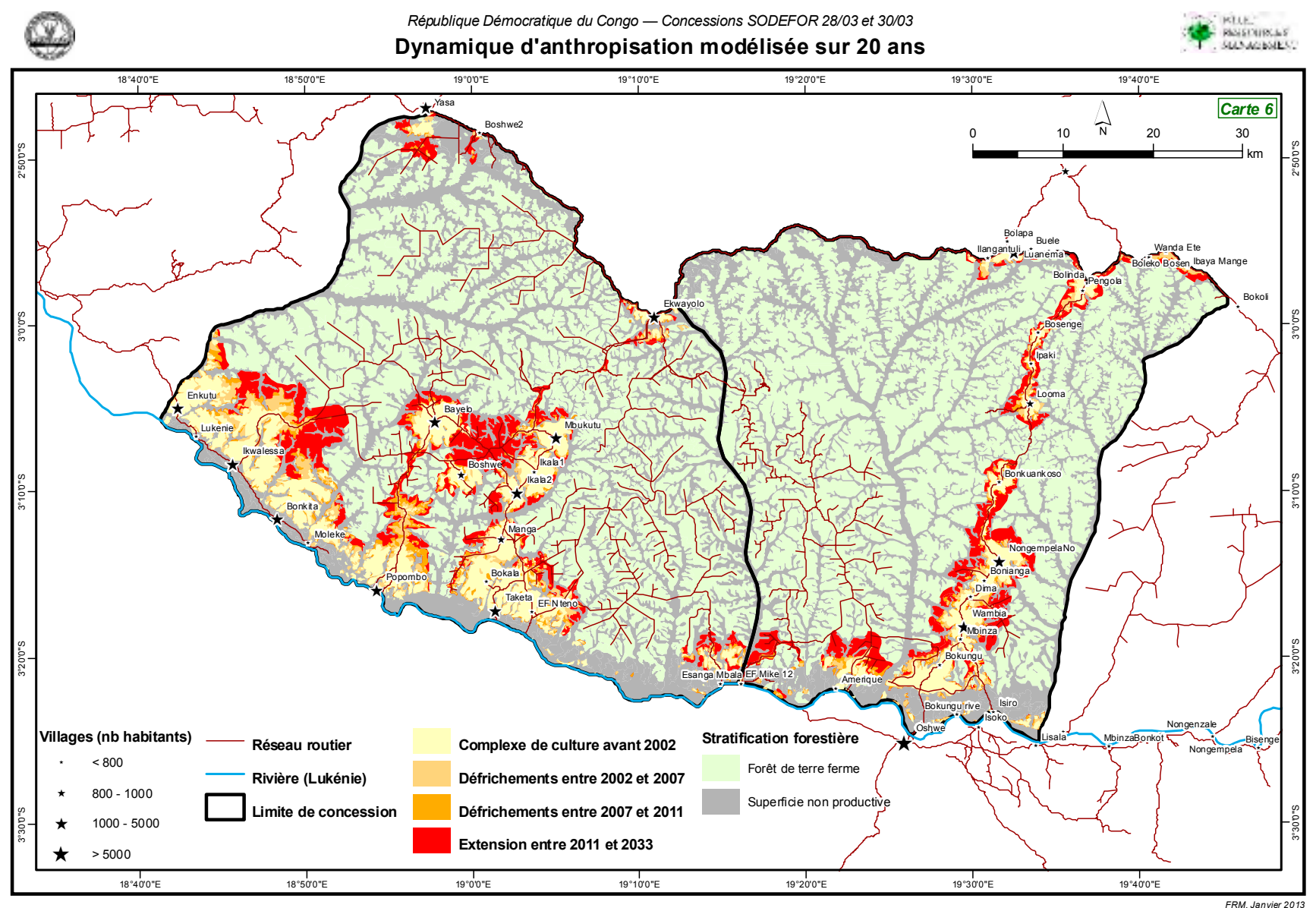

Carte 6 : Dynamique d'anthropisation modélisée sur 20 ans

Source: FRM 2013

estimée dans le scénario de référence. Les résultats sont indiqués dans le Tableau 1.

Il s'agit donc ici du scénario de référence, ou baseline : c'est-à-dire ce qu'il se passerait probablement si aucun projet proposant d'autres solutions à l'agriculture sur brûlis pour freiner la déforestation n'était mis en place. La partie suivante décrit les activités de REDD qui pourraient être développées en ce sens, et estime dans quelle mesure la déforestation pourrait être réduite de cette manière.

\subsection{Scénario de projet : activités prévues, quantification}

Les activités proposées dans le cadre du Volet 1 ne sont encore que des pistes de réflexion : avant dêtre mises en œuvre, elles devront être validées et précisées sur le terrain.

La principale raison à la pratique d'une agriculture pionnière semble être la perte de fertilité du milieu, liée à la podzolisation très rapide des sols, qui pousse les agriculteurs à cultiver des superficies importantes et à adopter de longues durées de mise en jachère. Pour répondre à ce phénomène, il convient de mettre en place des pratiques de fertilisation et de protection des sols contre l'érosion et le lessivage. La fertilisation chimique sur sol sableux est délicate à gérer et exige une connaissance très précise des besoins des cultures afin que les apports en fertilisants soient immédiatement consommés par les plantes cultivées, sans avoir le temps d'être lessivés par la pluie. Les agriculteurs de la région ne possèdent pas les connaissances ni les instruments de mesure pour appliquer des fertilisants chimiques de manière efficace, rentable et non polluante. L'enclavement de la zone, accessible uniquement par voie fluviale et peu desservie, est un autre obstacle à l'apport de fertilisants chimiques. La fertilisation organique permet un apport en éléments nutritifs, une amélioration du complexe argilo-humique, ainsi qu'une protection mécanique du sol contre la pluie.

L'agroforesterie offre des perspectives intéressantes, elle pourrait permettre la fertilisation des parcelles et leur fixation par la plantation de pérennes. Le manioc pourrait être intercalé entre les lignes d'une fabacée ligneuse locale à croissance rapide, par exemple 
Tableau 1 : Émissions de $\mathrm{CO}_{2}$ dans le scénario de référence

\begin{tabular}{|c|c|c|c|c|}
\hline $\begin{array}{l}\text { Années écoulées } \\
\text { depuis le début de la } \\
\text { période historique de } \\
\text { référence }\end{array}$ & Année & $\begin{array}{l}\text { Superficie des zones } \\
\text { anthropisées sur les } \\
\text { concessions } 28 / 03 \text { et } \\
30 / 03 \text { (historique réel } \\
\text { et projection) (ha) }\end{array}$ & $\begin{array}{l}\text { Superficie } \\
\text { déforestée annuelle } \\
\text { (ha) (scénario de } \\
\text { référence) }\end{array}$ & $\begin{array}{l}\text { Émissions annuelles } \\
\text { de } \mathrm{CO}_{2}\left(\mathrm{tCO}_{2} \mathrm{e}\right) \\
\text { (scénario de } \\
\text { référence) }\end{array}$ \\
\hline 0 & 2002 & 32662 & - & \\
\hline 1 & 2003 & 33956 & 1294 & 581488 \\
\hline 2 & 2004 & 35251 & 1294 & 581488 \\
\hline 3 & 2005 & 36545 & 1294 & 581488 \\
\hline 4 & 2006 & 37840 & 1294 & 581488 \\
\hline 5 & 2007 & 39134 & 1294 & 581488 \\
\hline 6 & 2008 & 40429 & 1294 & 581488 \\
\hline 7 & 2009 & 41723 & 1294 & 581488 \\
\hline 8 & 2010 & 43018 & 1294 & 581488 \\
\hline 9 & 2011 & 44312 & 1294 & 581488 \\
\hline 10 & 2012 & 45607 & 1294 & 581488 \\
\hline 11 & 2013 & 46901 & 1294 & 581488 \\
\hline $12\left(^{*}\right)$ & $2014(*)$ & 48196 & 1294 & 581488 \\
\hline 13 & 2015 & 49490 & 1294 & 581488 \\
\hline 14 & 2016 & 50785 & 1294 & 581488 \\
\hline 15 & 2017 & 52079 & 1294 & 581488 \\
\hline 16 & 2018 & 53374 & 1294 & 581488 \\
\hline 17 & 2019 & 54668 & 1294 & 581488 \\
\hline 18 & 2020 & 55963 & 1294 & 581488 \\
\hline 19 & 2021 & 57257 & 1294 & 581488 \\
\hline 20 & 2022 & 58552 & 1294 & 581488 \\
\hline 21 & 2023 & 59846 & 1294 & 581488 \\
\hline 22 & 2024 & 61141 & 1294 & 581488 \\
\hline 23 & 2025 & 62435 & 1294 & 581488 \\
\hline 24 & 2026 & 63730 & 1294 & 581488 \\
\hline 25 & 2027 & 65024 & 1294 & 581488 \\
\hline 26 & 2028 & 66319 & 1294 & 581488 \\
\hline 27 & 2029 & 67613 & 1294 & 581488 \\
\hline 28 & 2030 & 68908 & 1294 & 581488 \\
\hline 29 & 2031 & 70202 & 1294 & 581488 \\
\hline 30 & 2032 & 71496 & 1294 & 581488 \\
\hline 31 & 2033 & 72791 & 1294 & \\
\hline \multicolumn{2}{|c|}{ Total sur la période 2014-2033 } & & 25890 & 11629770 \\
\hline
\end{tabular}

(*) Début du projet REDD

le wenge (Millettia laurentii De Wild), dont des plantules peuvent être collectées en forêt. Ces haies seraient taillées régulièrement à moins d'un mètre de hauteur et les tailles seraient étalées sur le sol de manière à constituer un paillage de protection contre les pluies et un apport organique (Figure 2). 


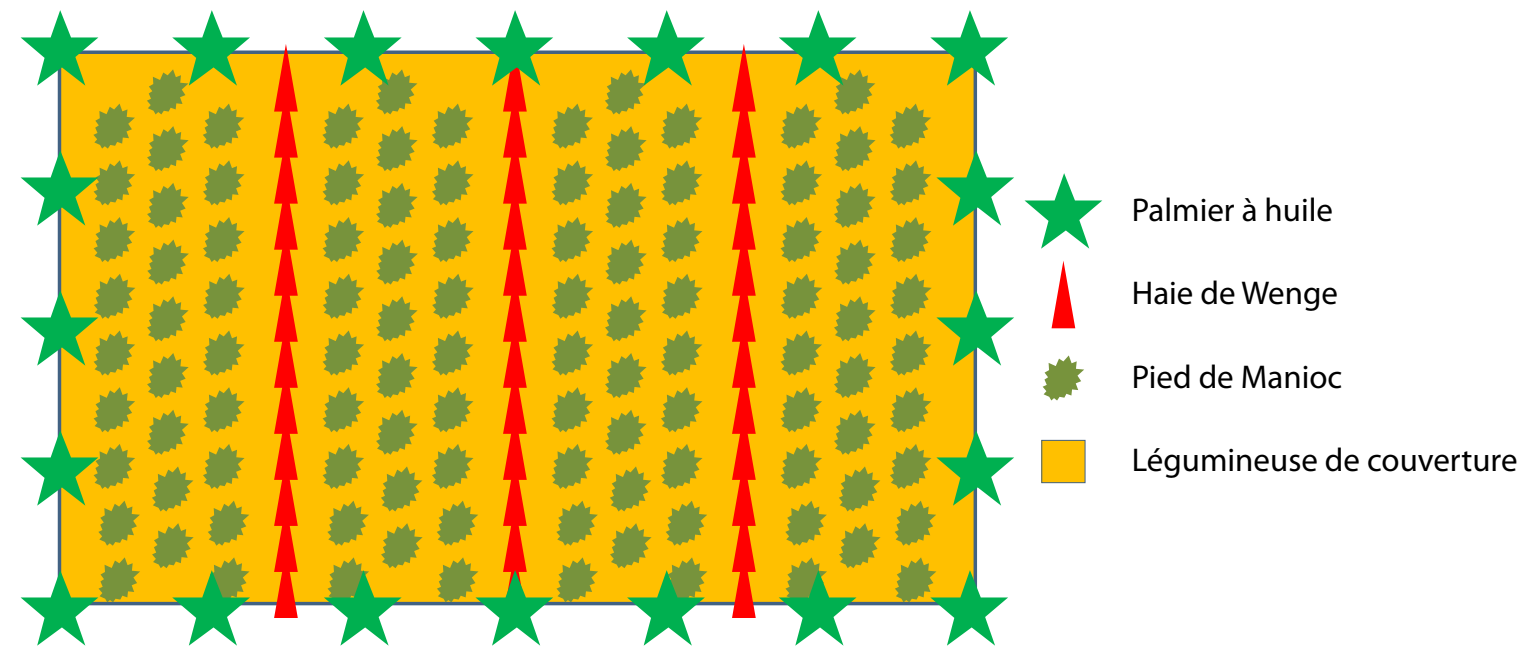

Figure 2 : Schéma d'une parcelle agroforestière

Source: Laurène Feintrenie, CIRAD, co-author of the report

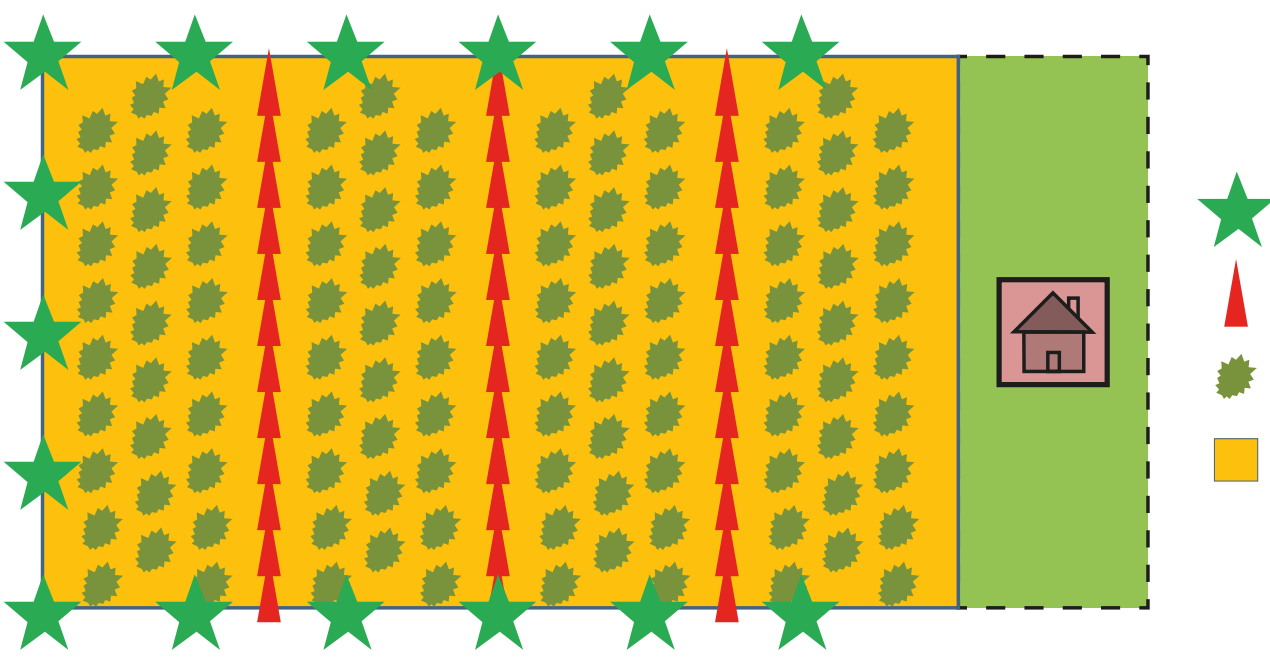

Palmier à huile

Haie de Wenge

Pied de Manioc

Légumineuse de couverture

Figure 3 : Schéma d'une parcelle agroforestière associée à un enclos et une bergerie Source: Laurène Feintrenie, CIRAD, co-author of the report

Une légumineuse de couverture pourrait être installée dès la mise en culture de la parcelle, de manière à protéger rapidement le sol de la pluie, à contribuer à la lutte contre les adventices, à participer à l'enrichissement du sol en azote et à la reconstitution d'une couche humifère. L'arachide (Arachis hypogaea) ou le niébé (Vigna unguiculata) peuvent être utilisés comme légumineuses de couverture en association avec le manioc. De nombreuses légumineuses pourraient également être testées, seules ou en mélange: Pueraria phaseolides, Mucuna cochinchinensis, Mucuna brachiata, Centrosema pubescens, Calopogonium mucunoides, Calopogonium caeruleum, Desmodium ovalifolium, etc. La culture d'une légumineuse de couverture peut nécessiter l'inoculation des semences avec des rhizobiums adaptés, en particulier si l'espèce utilisée n'est pas présente naturellement dans l'environnement. Des essais devront être réalisés pour trouver l'association la plus productive et la moins contraignante, et les espèces les mieux adaptées aux conditions pédo-climatiques.

Un élevage ovin pourrait être associé à ces parcelles agroforestières, de manière à faciliter l'apport en fumure. Dans le cas des «fermes" (champs implantés autour d'un campement isolé), l'habitation est proche du champ cultivé, il serait alors aisé de développer un petit élevage ovin en bout de champ (Figure 3). Cet élevage serait fait en enclos ou en stabulation afin de produire du fumier qui pourrait être facilement transporté dans les champs pour les fertiliser (actuellement, c'est l'élevage en divagation qui est pratiqué, ce qui ne bénéficie donc pas à la fertilisation des parcelles de manioc). Le développement de petits élevages permettrait de varier les sources de protéines 
des habitants, et peut-être de les détourner en partie de la chasse. Les animaux pourraient également être vendus pour apporter un revenu supplémentaire aux éleveurs.

La combinaison de pratiques agroforestières et de couverture du sol à un apport en fumure devrait permettre plusieurs récoltes de manioc successives sur une même parcelle, et peut-être une augmentation des rendements dans les récoltes successives.

L'utilisation de variétés de manioc résistantes à la mosaïque contribuerait à cette amélioration de la production. Il sera nécessaire de faire des essais avec suivi des rendements en manioc durant des récoltes successives avant de pouvoir conclure à la pérennisation de la culture. Â défaut de pérenniser complètement la culture du manioc sur la parcelle, une rotation consistant à alterner plusieurs années de manioc avec une jachère enrichie en Milletia laurentii pendant 4 à 8 ans pourrait être envisagée.

Dans le scénario de référence, une famille cultive traditionnellement 2 ha de manioc chaque année, avec en moyenne 0,5 ha par an défriché sur la forêt naturelle. Dans le scénario de projet, il pourrait ainsi être envisagé de sédentariser l'agriculture sur une surface fixe de 6 ha par famille, avec un cycle de 2 ans de culture du manioc suivi de 4 années seulement de jachère enrichie en légumineuses (voir Figure 4). Cet enrichissement permettrait la remise en culture des parcelles sans avoir à consommer et brûler de nouvelles superficies forestières chaque année. De plus, l'augmentation de la production agricole grâce aux techniques de fertilisation permettrait de combler les besoins alimentaires grandissants d'une population en pleine expansion.

Il faudrait tester la rotation permettant le meilleur enrichissement du sol : si la fertilisation apportée

\begin{tabular}{|l|c|c|c|c|c|c|}
\hline $\begin{array}{l}\text { Année de } \\
\text { culture }\end{array}$ & $\mathrm{i}$ & $\mathrm{i}+1$ & $\mathrm{i}+2$ & $\mathrm{i}+3$ & $\mathrm{i}+4$ & $\mathrm{i}+5$ \\
\hline champ 1 & $\mathrm{M} 1$ & $\mathrm{M} 2$ & $\mathrm{~J} 1$ & $\mathrm{~J} 2$ & $\mathrm{~J} 3$ & $\mathrm{~J} 4$ \\
\hline champ 2 & $\mathrm{J} 4$ & $\mathrm{M} 1$ & $\mathrm{M} 2$ & $\mathrm{~J} 1$ & $\mathrm{~J} 2$ & $\mathrm{~J} 3$ \\
\hline champ 3 & $\mathrm{J} 3$ & $\mathrm{~J} 4$ & $\mathrm{M} 1$ & $\mathrm{M} 2$ & $\mathrm{~J} 1$ & $\mathrm{~J} 2$ \\
\hline champ 4 & $\mathrm{J} 2$ & $\mathrm{~J} 3$ & $\mathrm{~J} 4$ & $\mathrm{M} 1$ & $\mathrm{M} 2$ & $\mathrm{~J} 1$ \\
\hline champ 5 & $\mathrm{J} 1$ & $\mathrm{~J} 2$ & $\mathrm{~J} 3$ & $\mathrm{~J} 4$ & $\mathrm{M} 1$ & $\mathrm{M} 2$ \\
\hline champ 6 & $\mathrm{M} 2$ & $\mathrm{~J} 1$ & $\mathrm{~J} 2$ & $\mathrm{~J} 3$ & $\mathrm{~J} 4$ & $\mathrm{M} 1$ \\
\hline
\end{tabular}

Figure 4 : Schéma de la rotation de 6 ans sur 6 ha, avec 1 cycle de manioc suivi de 4 ans de jachère Source: FRM 2013 par les pratiques agroforestières et la fumure le permettent, une succession de deux cycles (4 ans), voire trois cycles (6 ans), de manioc pourrait être envisagée, suivie de 6 à 8 ans de jachère enrichie en légumineuses. Finalement, selon les résultats des pratiques d'enrichissement du sol par l'agroforesterie et la fumure, une famille pourrait avoir besoin de 4 à $\mathbf{6}$ ha de terres seulement pour cultiver le manioc et les plantes associées, sans avoir à consommer de nouvelles superficies sur la forêt chaque année, et pour une production annuelle équivalente, voire meilleure, que les productions actuellement constatées.

La récolte de bois de feu dans les champs de manioc défrichés annuellement devra être compensée par une production de bois de feu hors du champ. Si les jachères enrichies ne suffisent pas, des plantations devront être envisagées dans un espace proche de l'habitat, à vocation de production de bois de feu.

Afin de développer ces nouvelles techniques agricoles, des animateurs locaux s'installeront dans

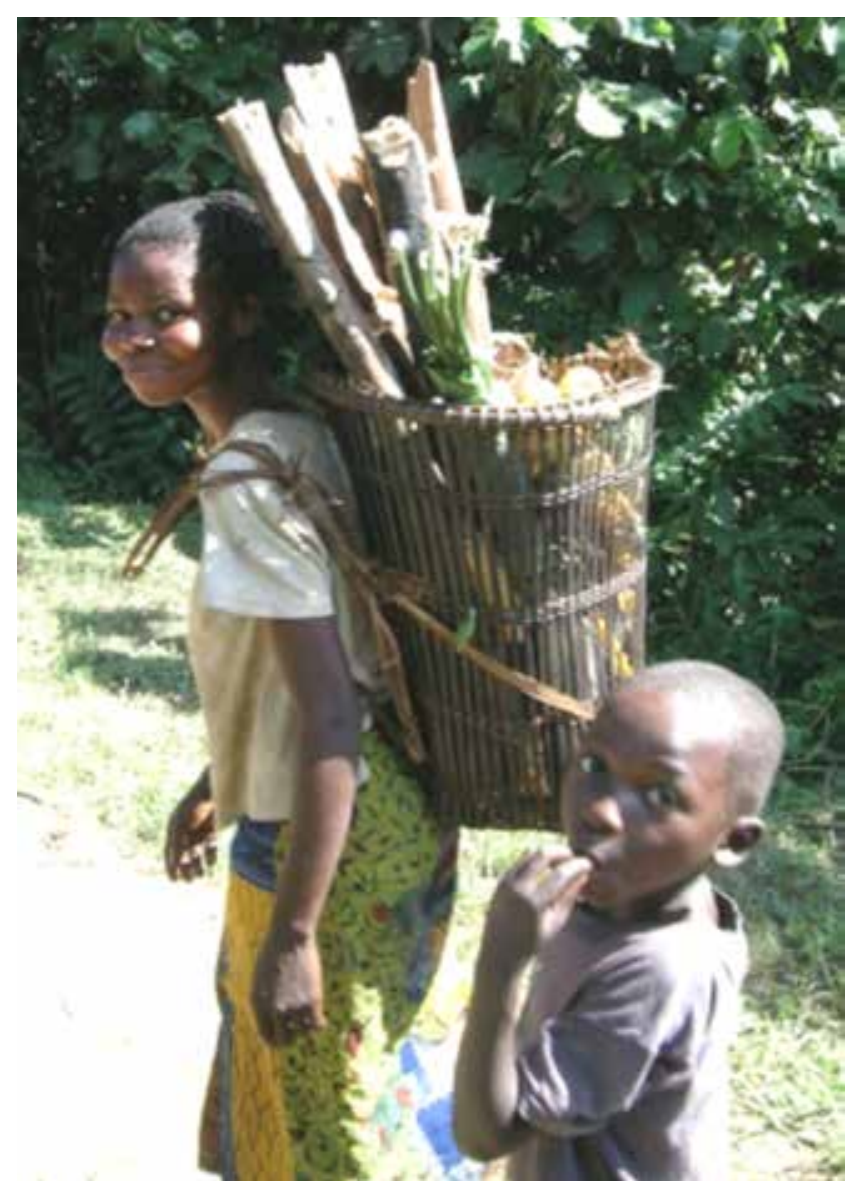

De retour des champs : femme transportant du bois de feu et des tubercules de manioc Source: CIRAD 
les villages de la zone d'étude et accompagneront les agriculteurs pendant toute la durée du projet, à raison d'un animateur en charge de 4 ou 5 villages : le développement des pratiques agroforestières et de fumure nécessitera en effet la formation des agriculteurs, la mise en place de champs de démonstration, de pépinières pour fournir les plants et les boutures nécessaires aux populations, et d'une procédure de prophylaxie pour les ovins (campagnes de vaccination et suivi vétérinaire). Un village servirait d'abord de pilote puis le projet pourrait être progressivement étendu à l'intégralité des deux concessions, en fonction du succès des premières expériences. Ainsi, le taux de pénétration des nouvelles pratiques augmenterait au cours du projet et, par suite, le taux de diminution de la déforestation également.

L'élevage en stabulation, en particulier, sera une nouvelle pratique qui demandera un suivi attentif de la part des animateurs : il ne sera donc testé que dans un nombre limité de villages pendant toute la durée du projet. Par ailleurs, avant de développer l'élevage ovin, il est indispensable de conduire une étude sur le potentiel de production d'un tel élevage, sur les risques d'épidémie et sur les besoins importants en prophylaxie. Des recommandations techniques précises devront être faites puisqu'il s'agit de développer des pratiques jusqu'alors non employées par les agriculteurs de la région.

Finalement, ces nouvelles techniques permettront d'améliorer la fertilité et la fixation des sols et, par suite, de favoriser le retour sur les parcelles précédemment exploitées après un temps limité (4 à 8 ans) de mise en jachère. Elles seront progressivement acceptées et pratiquées par les agriculteurs en fonction de leur efficacité et du dynamisme des animateurs. Ainsi, si l'on peut difficilement espérer réduire fortement la déforestation en début de projet (hypothèse considérée de $1 \%$ la deuxième année du projet), on peut en revanche espérer atteindre près de $75 \%$ d'efficacité (c'est-à-dire diminuer de $75 \%$ la déforestation annuelle) la dernière année du projet, lorsque ces pratiques seront bien intégrées par la population locale, soit une superficie défrichée de 324 ha la dernière année du projet au lieu des 1294 ha prévus par le scénario de référence. Les résultats sont résumés dans le graphique de la Figure 5 et dans le Tableau 2.

De ce différentiel de déforestation découle un différentiel d'émissions de $\mathrm{CO}_{2}$ (par un système de calcul identique à celui exposé dans la partie précédente), aboutissant à des émissions évitées de 3,5 millions de tonnes de $\mathrm{CO}_{2}$ e sur toute la durée du projet (20 ans). Les chiffres annuels sont repris dans le Tableau 2 ci-dessous.

Si le projet vient à être enregistré, le différentiel de déforestation devra être calculé entre le taux de déforestation observé du scénario de projet et le taux de déforestation estimé du scénario de référence, un scénario de référence qui devra être régulièrement calibré au regard de la zone géographique de référence (ou «zone témoin », située en dehors de la zone de projet).

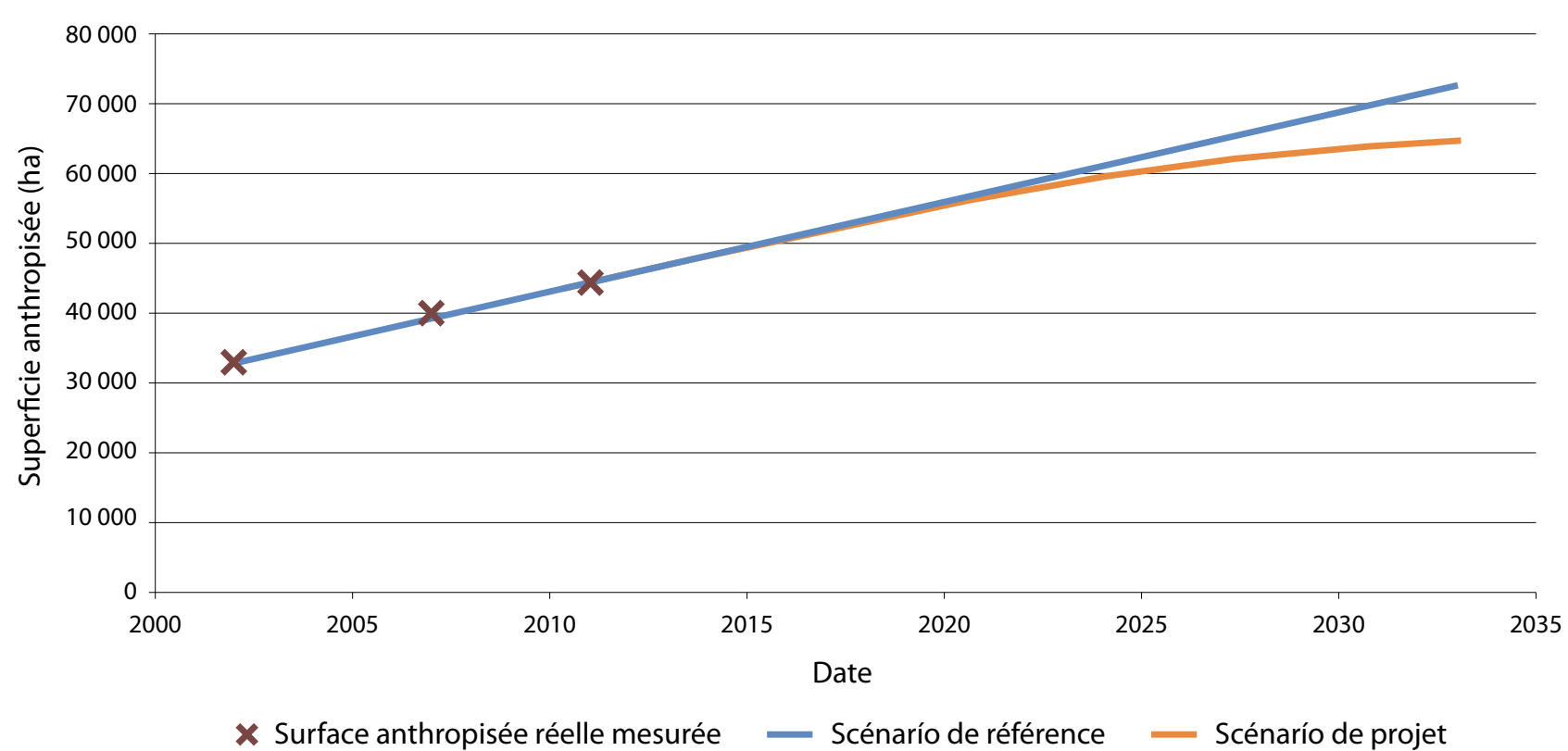

Figure 5 : Modélisation des superficies anthropisées sur les concessions 28/03 et 30/03 


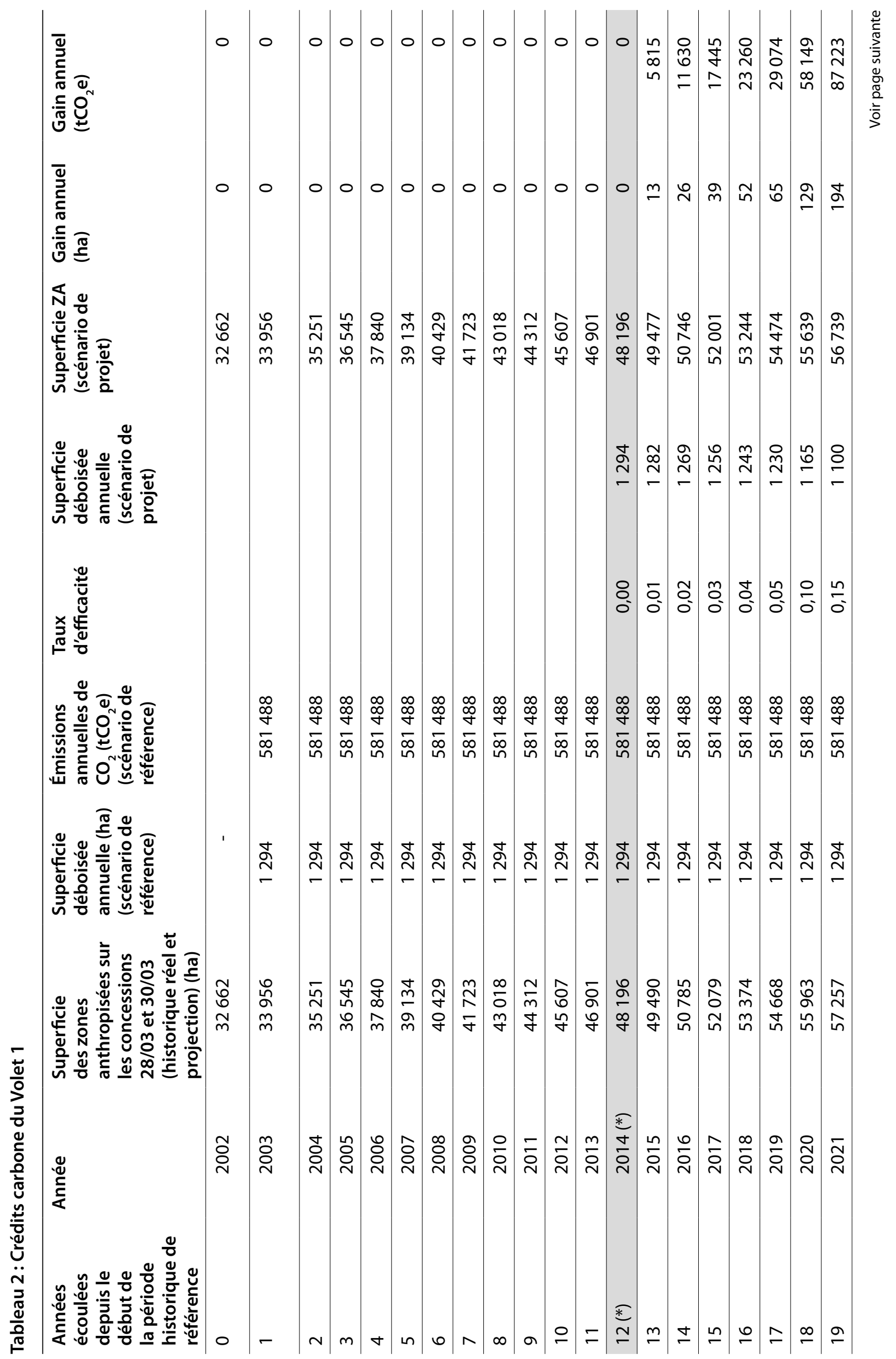




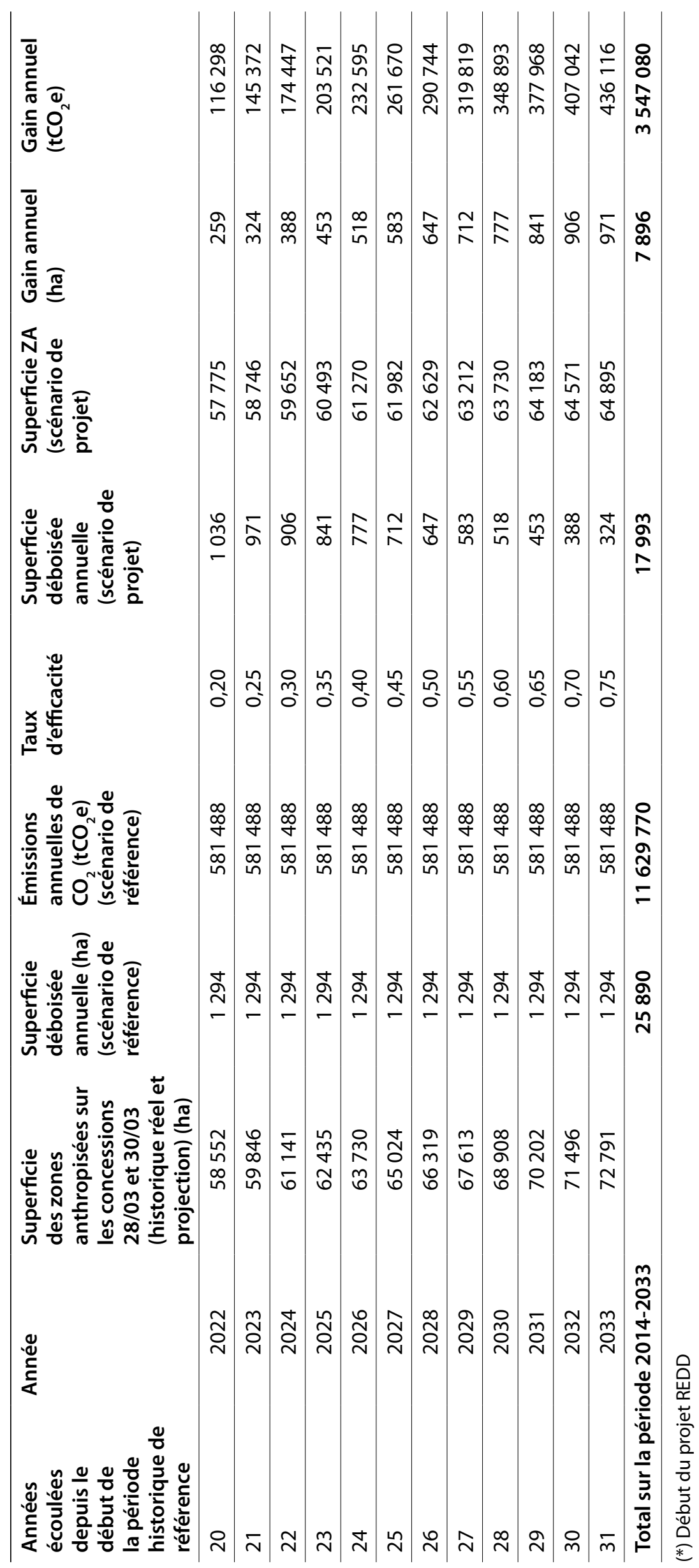


En plus des bénéfices carbone quantifiés ci-dessus, il est indéniable que le projet entraînera également d'importants bénéfices environnementaux (préservation de la forêt naturelle) et sociaux, grâce à la mise en place de techniques agricoles moins coûteuses en temps et en énergie que l'abattis et le brûlis traditionnellement pratiqués, ce qui permettra aux familles de développer d'autres activités. De plus, la productivité des parcelles agricoles devrait être sensiblement augmentée. Ces nouvelles pratiques agricoles devraient donc notablement améliorer les conditions de vie des populations locales.

Il faut néanmoins garder à l'esprit que toute amélioration de la commercialisation des produits agricoles ainsi obtenus peut à long terme induire la formation d'un bassin d'attraction pour les producteurs. L'objectif principal du projet étant de lutter contre la déforestation, il faut donc essayer de fixer l'agriculture et d'en améliorer la rentabilité, sans pour autant créer une agriculture marchande très profitable. Cela risquerait en effet de créer une forte valorisation de la terre et d'attirer davantage d'agriculteurs dans la région, augmentant ainsi la pression sur la forêt.

\subsection{Système de suivi}

Plusieurs systèmes de suivi devront être mis en place pour cette activité. Il faudrait, d'une part, mesurer l'efficacité des nouvelles pratiques agricoles (grâce à une évaluation des rendements agricoles et du temps de rotation des cultures, notamment) et, d'autre part, suivre l'évolution de la déforestation et du stock de carbone forestier.

En effet, les pratiques agroforestières telles qu'elles ont été exposées ci-dessus sont novatrices pour cette région, or l'objectif de diminution de la déforestation dépend intégralement de leur efficacité. Il sera donc absolument nécessaire de suivre attentivement l'acquisition de ces pratiques par les populations et l'évolution des rendements agricoles, afin de s'adapter au mieux aux problèmes rencontrés pour proposer des solutions permettant d'atteindre les objectifs fixés.

Le protocole à mettre en place est relativement simple : un réseau de parcelles cultivées par des familles devrait être suivi, en plus d'une parcelle de démonstration (un champ de manioc) qui puisse être contrôlée quotidiennement. Les femmes cultivant les parcelles doivent être rémunérées pour peser de manière systématique tous les tubercules de manioc sortant de leur parcelle, ainsi que les autres produits agricoles lors des saisons de récolte. Il faudra venir recopier les données relevées par la famille de manière hebdomadaire, afin de vérifier que le relevé des données est régulièrement effectué ; ce sera également l'occasion de payer la cultivatrice. Ce travail pourra être réalisé par des animateurs agronomes : ces animateurs, financés par le projet, devront vivre au village pour favoriser la proximité avec les populations locales. Enfin, chaque parcelle devra être clairement identifiée et son historique précisément suivi (temps de mise en jachère, fréquence de mise en culture, etc.).

Par ailleurs, un suivi satellite sera nécessaire en complément afin d'étudier l'évolution de la déforestation. Pour cela, une acquisition d'images SPOT tous les trois ans sera programmée. Les images de haute résolution permettront de suivre avec précision l'étendue des défrichements à chaque intervalle de temps; elles sont disponibles gratuitement pour les porteurs de projet REDD+ en République démocratique du Congo. À partir du suivi surfacique de la déforestation, une estimation des stocks de carbone perdus pourrait être réalisée en se fondant sur les données de l'inventaire d'aménagement.

\section{5 Éléments d'analyse financière}

Le développement des pratiques agroforestières et de fumure nécessitera la formation des agriculteurs, la mise en place de champs de démonstration et la mise en place d'une procédure de prophylaxie pour les ovins (campagnes de vaccination et suivi vétérinaire). Pour augmenter les chances de succès, des boutures de manioc de variétés résistantes à la mosaïque pourraient être distribuées, tandis que des ovins pourront être donnés aux agriculteurs ayant construit des bergeries selon les conseils fournis.

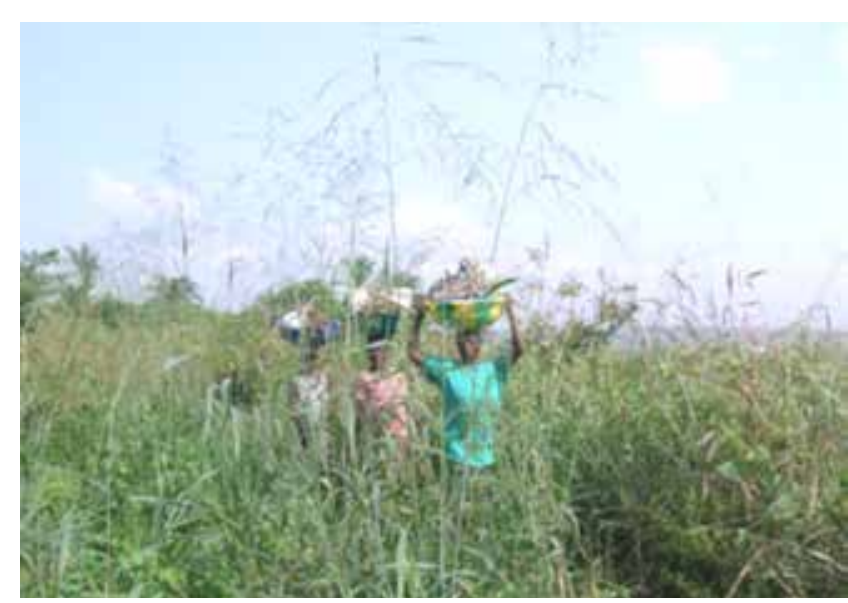

Femmes de retour des champs Source: CIRAD 
Cela nécessitera un effort continu dans la communication auprès des populations locales, ainsi que la présence d'animateurs agronomes au sein des villages des concessions pour diffuser ces nouvelles pratiques. Au début du projet, seuls un ou deux villages seront ciblés par le projet; puis, le nombre d'animateurs augmentera progressivement afin de toucher une part de la population de plus en plus importante.

Ce Volet 1 engendrant des bénéfices sociaux et environnementaux importants, le prix de vente de la tonne de $\mathrm{CO}_{2}$ pourrait également être élevé. Une question encore non résolue de ce Volet concerne justement la définition des titulaires des droits carbone. S'agit-il des populations locales? S'agitil du concessionnaire, porteur de projet ? S'agit-il de l'État, propriétaire foncier? D'autant plus que toute la zone définie comme étant susceptible d'être défrichée par l'agriculture vivrière (dans le scénario de référence) devrait être définie comme zone à vocation agricole dans le plan d'aménagement forestier, affectée au développement des activités agricoles des populations, et donc non exploitable par le concessionnaire. Cette zone sera donc exclue de la concession redéfinie par le Plan d'aménagement. Étant donné que les populations locales, par le changement de leurs pratiques agricoles, seront les acteurs principaux de la réduction de la déforestation, et que ces activités auront lieu dans la superficie affectée au développement rural, donc en dehors de la concession, on pourrait légitimement considérer que les populations locales seront titulaires des droits carbone. En parallèle, la SODEFOR étant porteur de projet, initiateur du projet et responsable de sa réussite, on peut également légitimement considérer que le concessionnaire devra être rémunéré pour son engagement dans la mise en œuvre de ce projet. Finalement, la question du bénéficiaire des crédits carbone demande véritablement à être éclaircie ; on peut supposer que le bénéfice des crédits carbone délivrés par le projet pourrait être partagé entre les différents acteurs (populations locales, concessionnaire et État, notamment) selon des proportions qui restent à définir. Autrement, un contrat signé entre le porteur de projet et l'État pourrait stipuler que le porteur de projet est le propriétaire exclusif des droits carbone, et qu'il serait ensuite de sa responsabilité de partager les revenus issus de la vente des crédits carbone entre les différents acteurs. Ainsi, les revenus qui reviendront aux populations locales pourraient être reversés par le biais d'un fonds de développement local (un fonds

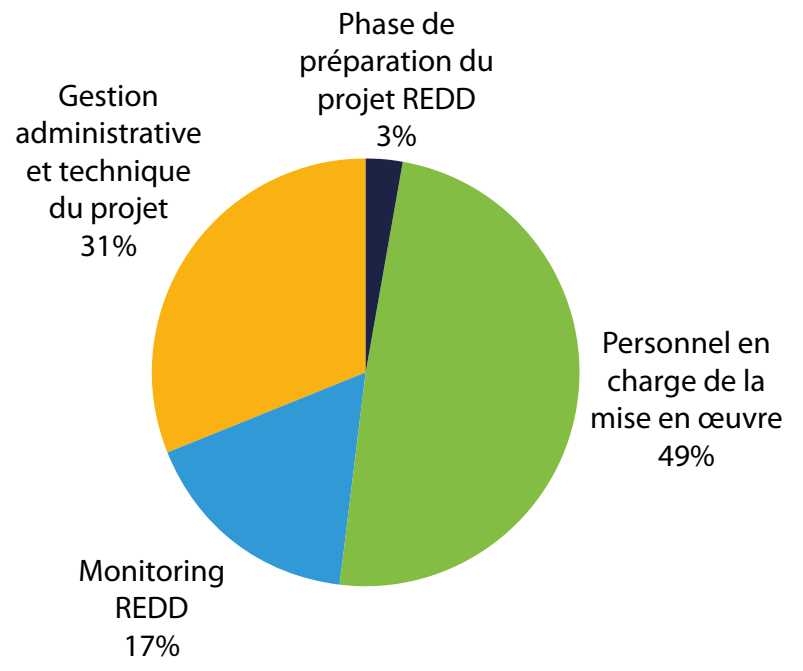

Figure 6 : Ventilation du budget total du Volet 1 dans les différentes rubriques

Source: FRM 2013

par village ou groupe de villages) géré directement par un comité constitué de représentants villageois et chargé de financer des infrastructures et des projets d'intérêt général (écoles, infrastructures de santé, routes de désenclavement, etc.). Ce système est déjà appliqué et opérationnel dans le cadre des accords constituant les clauses sociales des cahiers des charges, négociés entre le concessionnaire et les populations villageoises préalablement à la signature des contrats de concession, en application du Code forestier ${ }^{8}$. Il pourrait ici être contractualisé sous forme d'ERPA (contrats d'achat de réduction d'émissions).

Une estimation détaillée du budget nécessaire à la mise en œuvre du Volet 1 a été réalisée, dont le total s'élèverait à 13,3 millions d'euros sur 20 ans, soit en moyenne près de 665000 euros/an. Le Tableau 9 cidessous indique la répartition des montants selon les différents postes budgétaires.

Le poste budgétaire le plus important concerne le personnel en charge de la mise en ouvre, c'est-àdire les charges associées aux animateurs agronomes envoyés dans les villages et chargés de la diffusion des nouvelles pratiques, incluant le matériel nécessaire. Le deuxième poste budgétaire concerne la gestion administrative et technique du projet : l'encadrement technique et administratif, la location d'un bureau

8 Le cahier des charges est une annexe du contrat de concession, il comprend une « clause particulière relative à la réalisation d'infrastructures socio-économiques au profit des communautés locales " (article 89 de la loi 011/2002 portant Code forestier). 
(dont les charges seront partagées avec les deux autres volets) et le financement de missions à court terme d'experts agronomes internationaux. Enfin, $20 \%$ du budget sont consacrés aux dépenses directement liées au carbone (enregistrement du projet, suivi des stocks de carbone).

Le coût de revient de la tonne de carbone dans le Volet 1 s'élève ainsi à 3,70 euros/tCO 2 e. En proposant un prix de vente de 5 euros $/ \mathrm{tCO}_{2}$, le projet pourrait engendrer des bénéfices à partager entre les différents acteurs tout en étant concurrentiel sur le marché international du carbone. Choisir un standard de qualité permettrait de mettre en valeur les nombreux bénéfices socio-économiques du projet (voir 6.2 Bénéfices sociaux et environnementaux p. 70).

Par ailleurs, il est à noter que l'émission de crédits carbone étant très progressive (peu de crédits en début de projet), le besoin de financement sera important au démarrage du projet. 


\section{Volet 2 : exploitation forestière à impact réduit}

\section{1 État initial}

Le Ministère de l'environnement, conservation de la nature, eaux et forêts de République démocratique du Congo a mis à disposition du public un guide opérationnel définissant les normes d'exploitation forestière à impact réduit (EFIR). Ces normes sont progressivement intégrées dans toutes les concessions de la SODEFOR ; la SODEFOR est également entrée dans une dynamique de certification FSC.

La SODEFOR se trouve donc dans un processus d'amélioration de ses techniques d'exploitation, mais celles-ci peuvent être encore optimisées dans l'optique d'une amélioration de l'évolution des stocks de carbone forestier, la problématique étant la suivante : comment limiter la diminution des stocks de carbone forestier liés à l'exploitation forestière, sans diminuer la production de bois? Dans ce Volet 2 du projet pilote REDD+ de la Lukénie, c'est sur la desserte forestière que se concentreront les efforts d'amélioration.

La desserte forestière est construite essentiellement pour l'évacuation des grumes exploitées. Sur les concessions de la SODEFOR, il existe différents types de routes et pistes :

- Les routes principales ou permanentes assurent une circulation intensive des grumiers durant une longue période (plusieurs années). Elles doivent être suffisamment larges pour permettre les croisements des véhicules (7-8 $\mathrm{m}$ de plateforme) et ensoleillées pour faciliter le séchage du sol après les pluies et ainsi éviter l'embourbement des véhicules.

- Les routes secondaires ont une durée d'utilisation de quelques mois. Moins larges, de 4 à $6 \mathrm{~m}$ de chaussée, elles sont également ensoleillées.

- Les pistes de débardage permettent le transport de la grume, de l'emplacement de l'arbre sur pied jusqu'au parc de stockage en bord de route secondaire. Créées directement par le tracteur de débardage (skidder) lors de son déplacement jusqu'à l'arbre à abattre, elles n'excèdent pas $4 \mathrm{~m}$ de large et n'ont pas d'incidence notable sur la canopée.

Ce réseau permet donc de transporter les grumes à partir de l'emplacement de l'arbre sur pied jusqu'au "beach" situé sur les rives de la Lukénie ; les grumes sont alors assemblées en radeaux pour flotter, ou chargés sur des barges, jusqu'à Nioki ou Kinshasa où se trouvent les sites industriels.

La vue transversale d'une route principale ou secondaire peut être schématisée ainsi (Figure 7) :

Ainsi, sur la bande de roulement, tous les arbres sont abattus pour permettre le passage des véhicules.

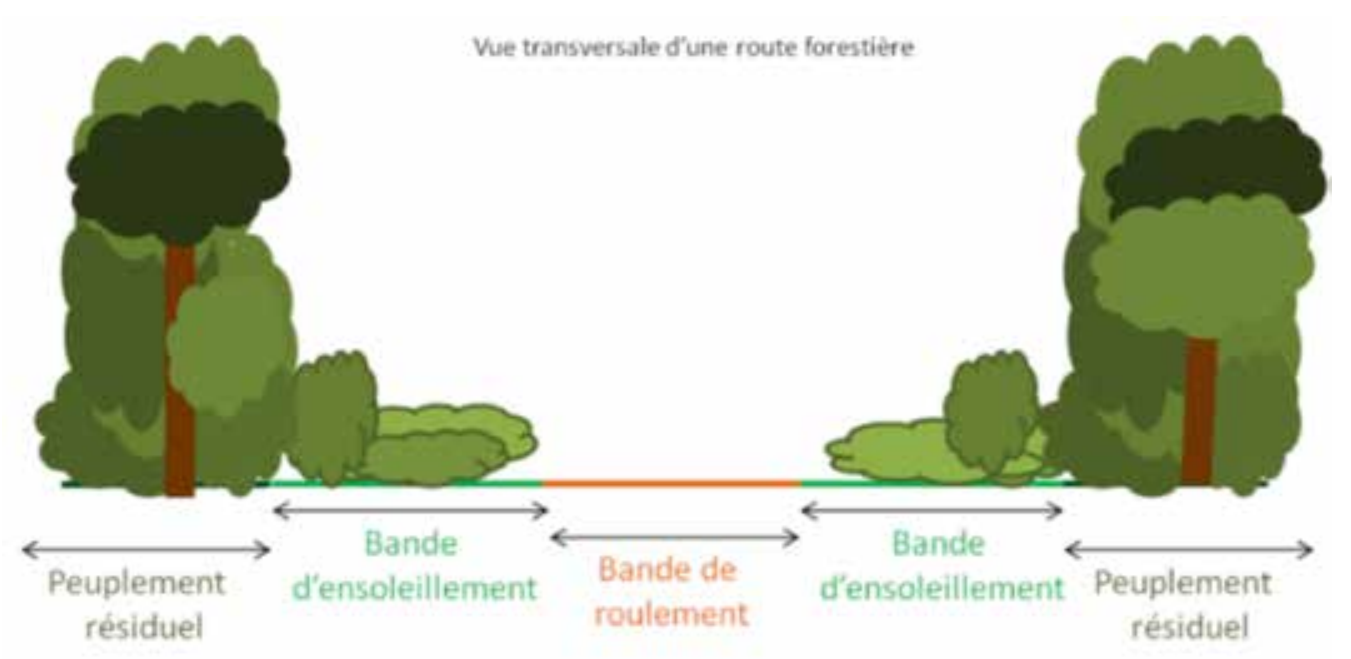

Figure 7 : Vue transversale d'une route forestière

Source: FRM 2013 
De plus, de part et d'autre de la bande de roulement se trouvent des bandes d'ensoleillement qui servent à assurer un bon séchage de la route après les pluies. Ainsi, de chaque côté de la route, tous les arbres qui risquent de projeter de l'ombre sur la bande de roulement sont abattus. Les bandes d'ensoleillement sont donc principalement constituées de végétation buissonnante et de petits arbres, et le stock de carbone y est particulièrement faible par rapport au peuplement résiduel. D'après les normes nationales, l'emprise totale d'une route (bande de roulement et bandes d'ensoleillement) ne doit pas excéder $30 \mathrm{~m}$ de large.

Les pistes de débardage sont quant à elles formées par le skidder lors de ses déplacements entre les tiges à abattre et la route secondaire. Le réseau de pistes de débardage est déjà optimisé de manière à limiter les passages d'engin. De plus, les pistes étant créées par le skidder à l'aide de sa pelle frontale, aucune tige de plus de $20 \mathrm{~cm}$ de diamètre ne peut être abattue : seules les petites tiges et la végétation non ligneuse (marantacées, etc.) sont affectées par la création d'une piste de débardage, sur environ 4 mètres de large ; la création d'une piste de débardage présente donc un bilan carbone moins défavorable que la création d'une route secondaire. Actuellement, la longueur d'une piste de débardage n'excède pas $500 \mathrm{~m}$; c'està-dire que le réseau de routes secondaires est planifié de manière à être situé à moins de $500 \mathrm{~m}$ des arbres à abattre.

Il apparaît donc principalement trois leviers pour améliorer le bilan carbone de la desserte forestière sans baisse de productivité :

- La largeur des routes (et plus particulièrement des bandes d'ensoleillement);

- L'intensité des coupes en bord de route lors de la formation des bandes d'ensoleillement ;

- La longueur des routes secondaires (en substituant les tronçons terminaux des routes secondaires par des pistes de débardage).

\section{2 Élaboration du scénario de référence}

Aucune méthodologie REDD+ n'a été établie à ce jour pour estimer les réductions d'émissions liées au changement de méthode d'exploitation forestière (passage des méthodes conventionnelles d'exploitation à des méthodes d'exploitation forestière à impact réduit (EFIR)). La méthodologie utilisée ici est donc expérimentale et devra être validée si le projet est développé.
Un projet REDD consiste à évaluer la réduction des émissions de gaz à effets de serre entre un scénario de projet et un scénario de référence, le scénario de référence correspondant à ce qu'il se passerait si aucun projet de réduction d'émissions n'était mis en place et si les pratiques continuaient au fil de l'eau (business as usual), dans la ligne de ce qui se faisait historiquement. La question qui se pose ici est donc le choix du scénario de référence, dont dépend grandement l'estimation de la réduction d'émissions (et donc de la quantité de crédits carbone délivrés). Le scénario de référence doit-il se fonder sur les pratiques actuelles du concessionnaire, avant la mise en place du projet REDD ? Ou doit-il se baser sur les normes nationales, afin de proposer un scénario de référence qui serait valable pour tous les concessionnaires de République démocratique du Congo ? Finalement, ces deux options ont été étudiées et sont présentées ci-dessous.

Une mission de terrain a été menée afin de mettre en place un protocole de mesures de largeur de bandes de roulement, de largeur de bandes d'ensoleillement et de stock de biomasse sur les différents types d'infrastructures routières; ce protocole de terrain a été établi en partenariat avec Jean-Gaël Jourget (ingénieur chez FRM en charge de l'aménagement forestier des concessions SODEFOR), en concertation avec Lilian Blanc (chercheur au CIRAD).

\subsubsection{Mesures de largeur des routes forestières}

Le protocole de mesure de largeur des routes principales et secondaires peut être schématisé comme suit (Figure 8) :

Sur un transect de $50 \mathrm{~m}$ de longueur, neuf mesures perpendiculaires à l'axe de la route ont été effectuées : une mesure de la largeur de la bande de roulement à $0 \mathrm{~m}$ du point de départ (point GPS initial), une deuxième mesure à $25 \mathrm{~m}$ du point de départ et une dernière mesure à $50 \mathrm{~m}$. Aux mêmes intervalles, la largeur des bandes d'ensoleillement (à gauche et à droite) a été mesurée. Ces mesures ont été répétées sur une dizaine de transects couvrant différentes situations : routes primaires et secondaires, routes d'ouverture (nouvelles) ou de réouverture (de routes précédemment ouvertes).

La largeur de la bande de roulement s'est avérée peu variable : de 6,9 m en moyenne, elle oscille entre $6,0 \mathrm{~m}$ et $8,1 \mathrm{~m}$. Les bandes d'ensoleillement 


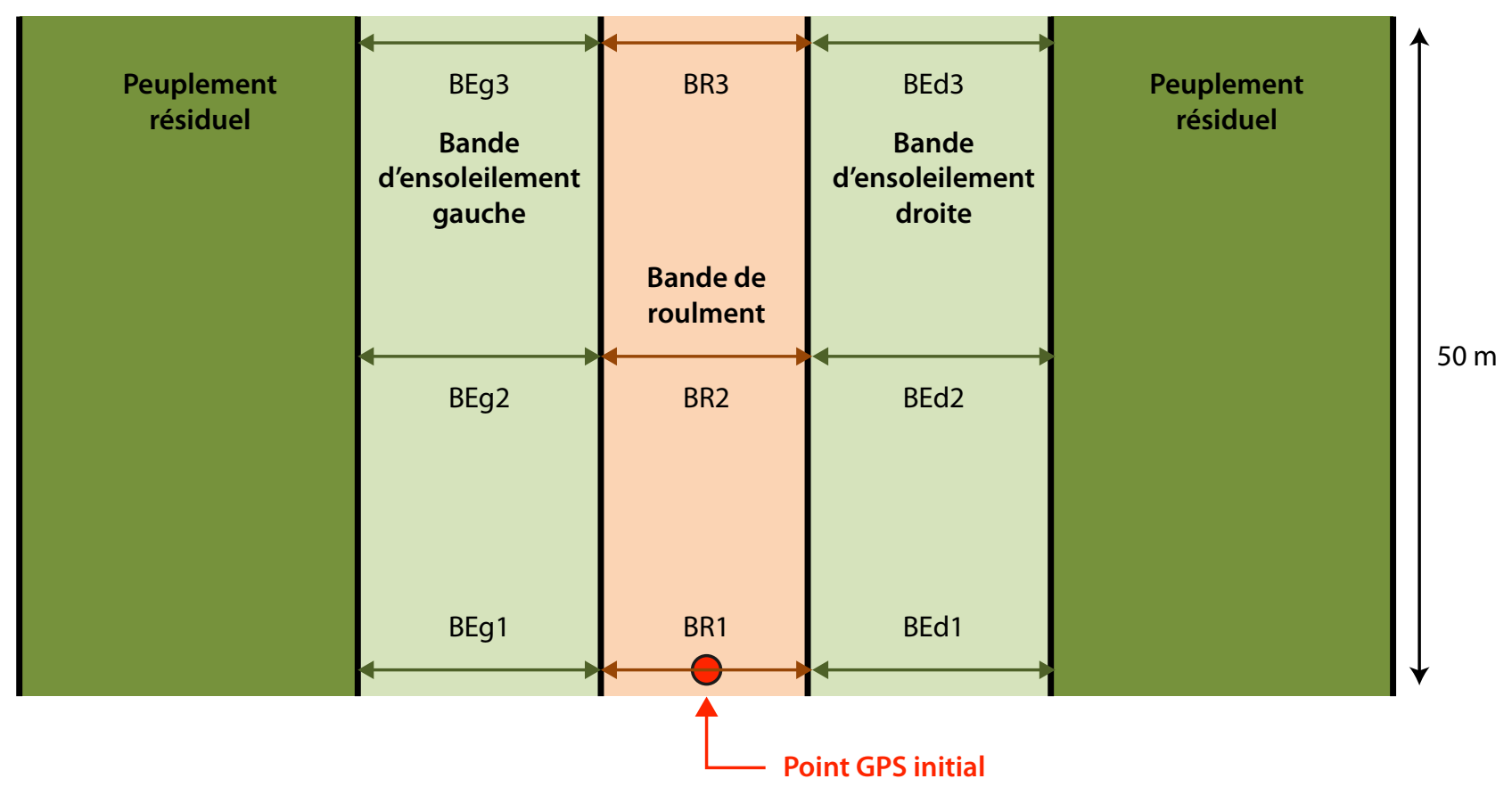

Figure 8 : Protocole de mesure de largeur des routes forestières

Source: FRM 2013

présentent au contraire une largeur très variable : de 13,1 m en moyenne, elles oscillent entre 4,1 m et $25,8 \mathrm{~m}$. Cette grande variabilité est liée au type d'ouverture (les routes de réouverture présentent un sol plus compacté, moins sensible aux ornières, et nécessiteraient donc des bandes d'ensoleillement moins larges que les routes d'ouverture), à la date d'ouverture (les anciennes routes, créées avant la mise en place des normes EFIR, ont des bandes d'ensoleillement plus larges) et possiblement à l'orientation de la route (l'ensoleillement semble moins important sur les routes est-ouest bénéficiant d'une grande durée d'ensoleillement). Mais cela nécessiterait d'autres répétitions pour confirmer ces premières tendances. Par ailleurs, il est à noter que les bandes d'ensoleillement sont souvent difficiles

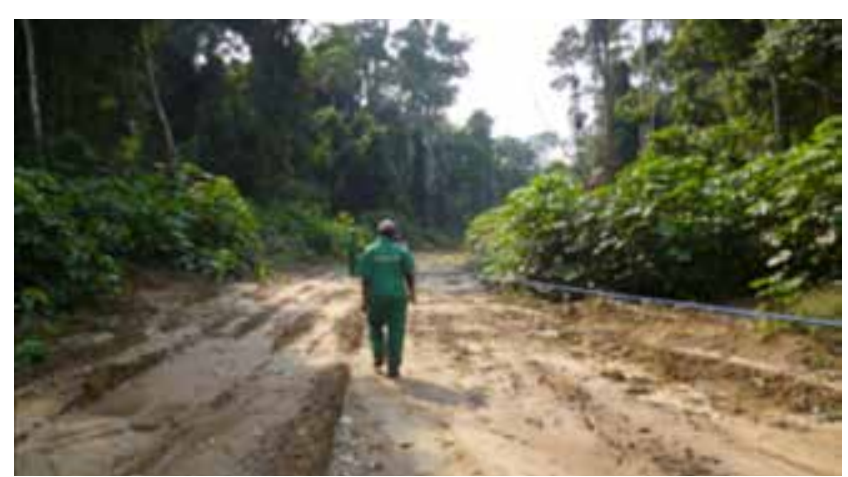

Mise en place du transect de mesure de biomasse sur une route secondaire récemment ouverte Source: FRM 2013 à délimiter, surtout sur les anciennes routes : il existe donc probablement un biais observateur non négligeable.

Finalement, l'emprise des routes forestière SODEFOR est de $32 \mathrm{~m}$ en moyenne (39 m pour les routes principales et $25 \mathrm{~m}$ pour les routes secondaires). L'emprise des routes tend donc à se rapprocher de la norme nationale EFIR, qui est de $30 \mathrm{~m}$ maximum.

\subsubsection{Mesure des stocks de biomasse}

Sur ces mêmes transects, la biomasse a également été mesurée. Pour cela, une corde a été tendue audessus de l'axe central de la route et tous les arbres présents à moins de $2,5 \mathrm{~m}$ de part et d'autre de la corde ont été inventoriés, soit des placettes de $250 \mathrm{~m}^{2}$ (voir Figure 9). La biomasse, évidemment, est généralement nulle sur les bandes de roulement. Par ailleurs, un transect équivalent a été mis en place en parallèle sur la bande d'ensoleillement de droite, à $5 \mathrm{~m}$ du bord de la route $(10 \mathrm{~m}$ pour les routes principales); toutes les essences de plus de $5 \mathrm{~cm}$ de diamètre ont été relevées.

L'essence et la classe de diamètre de toutes les tiges de plus de $5 \mathrm{~cm}$ de diamètre ont ainsi été relevées. Grâce à des équations allométriques et à la méthode expliquée dans la deuxième partie, il devient alors possible d'estimer le stock de carbone contenu 

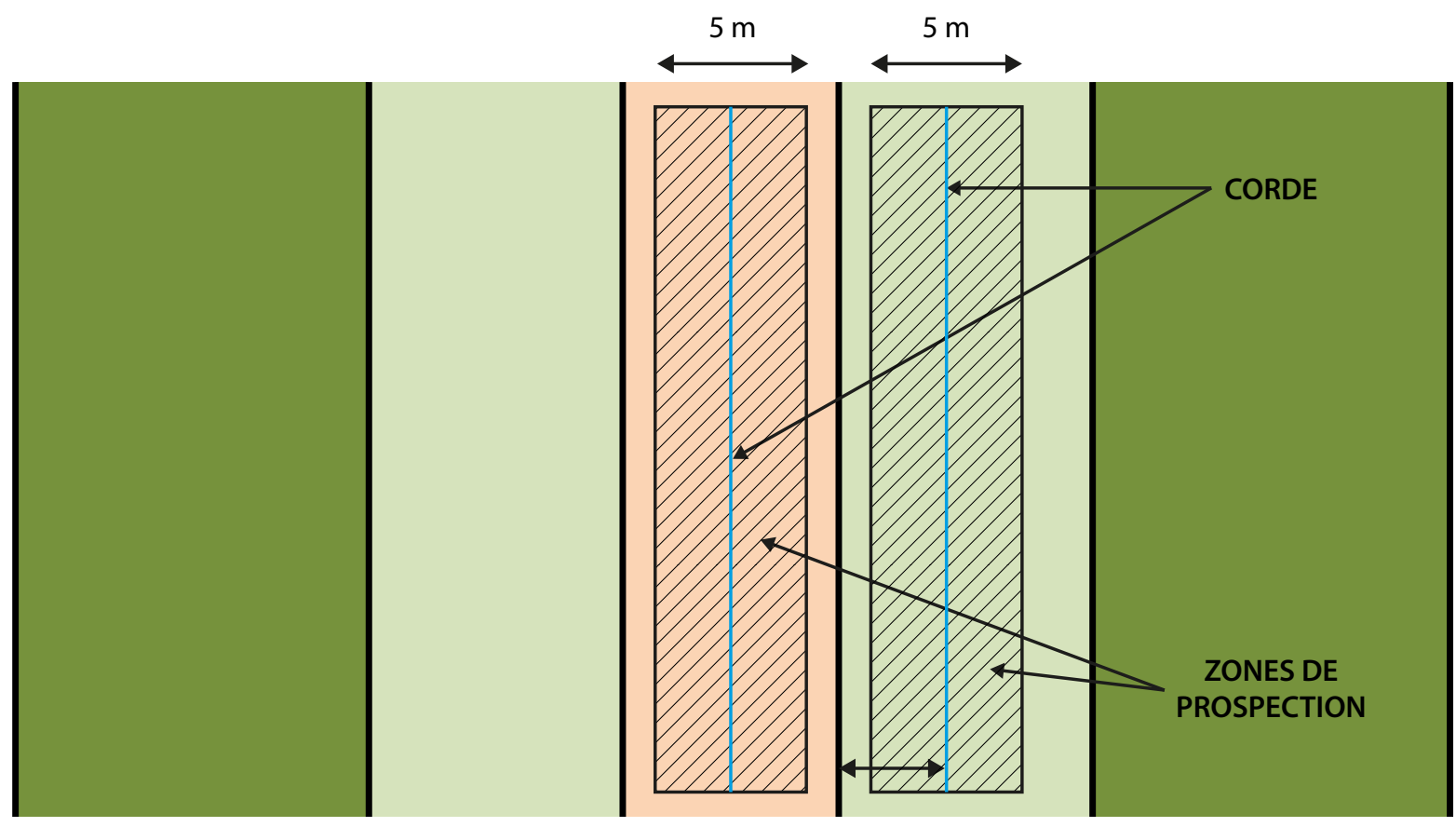

Eloignement du bord de route :

$5 \mathrm{~m}$ si route secondaire

$10 \mathrm{~m}$ si route principale

Figure 9 : Protocole de terrain : mesure des stocks de carbone forestier

Source: FRM 2013

dans la biomasse aérienne des différentes zones de prospection.

Finalement, il s'est avéré que le stock de biomasse à l'hectare sur les bandes d'ensoleillement, quelques mois après la création de la route, est d'environ $70 \mathrm{tC} / \mathrm{ha}$ (soit $257 \mathrm{tCO}_{2} / \mathrm{ha}$ ) et augmente avec l'âge de la route (reconstitution progressive de la biomasse sur les bandes d'ensoleillement). Mais la variabilité est grande d'une placette à l'autre et il serait nécessaire d'effectuer un grand nombre de répétitions pour avoir des données exploitables.

Ces mesures de terrain ont de plus montré que, après coupe, les bandes d'ensoleillement sont recolonisées rapidement par des essences pionnières (parasolier, macaranga, etc.) : il s'agit d'essences à croissance rapide, qui possèdent donc un bois de faible densité.
La biomasse aérienne est par conséquent faible, malgré l'apparente densité de la végétation. Il apparaît surtout que le stock de carbone des bandes d'ensoleillement est fortement dépendant des quelques gros arbres préservés lors de la création des bandes d'ensoleillement.

Il n'existe pas de normes nationales sur le mode de création des bandes d'ensoleillement : il n'est ainsi pas spécifié si l'intégralité de la végétation des bandes d'ensoleillement peut être détruite ou si une certaine proportion doit être préservée. Les pratiques actuelles seront donc retenues pour l'élaboration du scénario de référence : lors de l'ouverture d'une route, l'intégralité de la végétation présente en bord de route est détruite pour la création des bandes d'ensoleillement.

Tableau 3 : Emprise spatiale des routes dans le scénario de référence

\begin{tabular}{|c|c|c|c|c|c|}
\hline $\begin{array}{l}\text { BASELINE } \\
\text { (Pratiques actuelles) }\end{array}$ & Densité & $\begin{array}{l}\text { Bande de } \\
\text { roulement }\end{array}$ & $\begin{array}{c}\text { Bande } \\
\text { d'ensoleillement }\end{array}$ & Emprise totale & Surface affectée \\
\hline Routes principales & $5,9 \mathrm{~m} / \mathrm{ha}$ & $6,9 \mathrm{~m}$ & $16,2 \mathrm{~m}$ & $39,3 \mathrm{~m}$ & $229,9 \mathrm{~m}^{2} / \mathrm{ha}$ \\
\hline Routes secondaires & $6,0 \mathrm{~m} / \mathrm{ha}$ & $6,8 \mathrm{~m}$ & $9,0 \mathrm{~m}$ & $24,8 \mathrm{~m}$ & $148,3 \mathrm{~m}^{2} / \mathrm{ha}$ \\
\hline Total & $11,8 \mathrm{~m} / \mathrm{ha}$ & & & & $378,2 \mathrm{~m}^{2} / \mathrm{ha}$ \\
\hline
\end{tabular}


Scénario de projet : substitution des pistes principales et secondaires par des pistes de débardage à $1500 \mathrm{~m}$

Exemple du bloc d'exploitation $n^{\circ} 91$

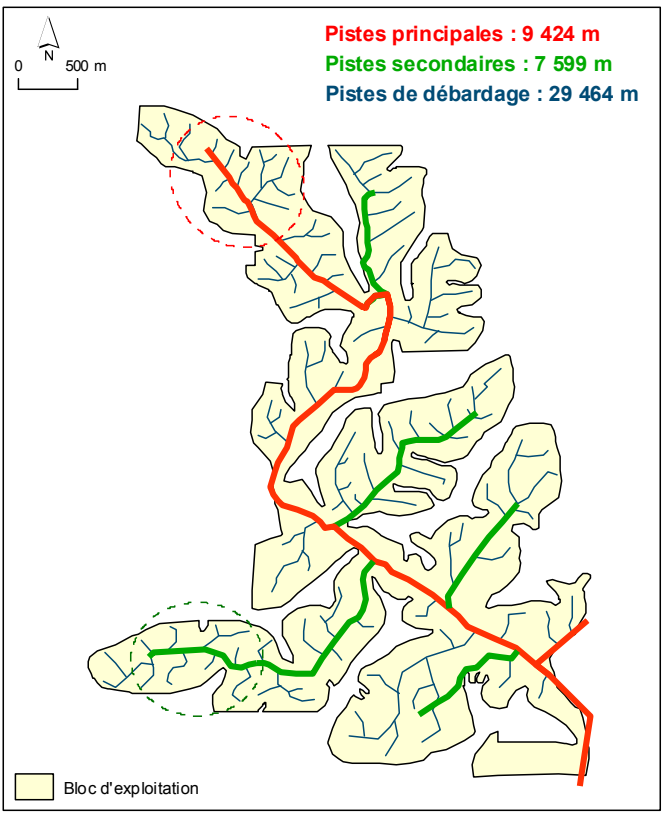

Scénario de référence :

réseau de pistes principales et de pistes secondaires + pistes de débardage à $500 \mathrm{~m}$

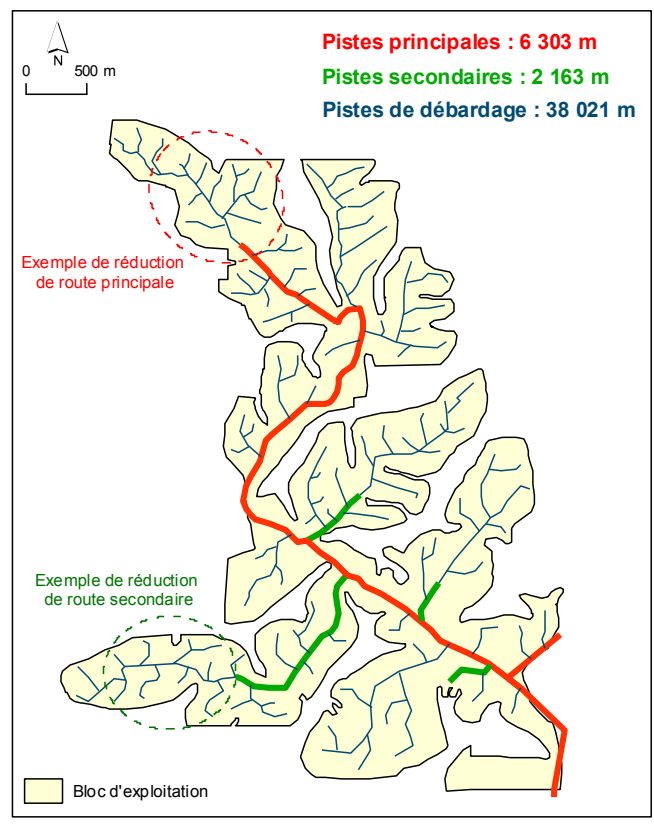

Scénario de projet :

réseau de pistes principales et de pistes secondaires + pistes de débardage à $1500 \mathrm{~m}$

- $3121 \mathrm{~m}$ de pistes principales

$-5436 \mathrm{~m}$ de pistes secondaires

$+8557 \mathrm{~m}$ de pistes de débardage

Carte 7 : Scénario de projet : substitution des pistes principales et secondaires par des pistes de débardage Source: FRM 2013

\subsubsection{Mesure de l'emprise spatiale du réseau routier}

Par ailleurs, le réseau routier a été relevé sur SIG pour différents blocs d'exploitation (voir l'exemple du bloc $\mathrm{n}^{\circ} 91$ sur la concession 28/03, Carte 7) ; il a ainsi été mesuré qu'il existe environ $5,9 \mathrm{~m} / \mathrm{ha}$ de routes principales et $6,0 \mathrm{~m} / \mathrm{ha}$ de routes secondaires. D'après les largeurs de routes mesurées, la surface affectée par les routes principales et secondaires est d'environ $378 \mathrm{~m}^{2} /$ ha ; elle est de $355 \mathrm{~m}^{2} / \mathrm{ha}$ en respectant l'emprise maximale de $30 \mathrm{~m}$ de large conseillée par les normes nationales. Ces données sont reprises dans les tableaux ci-dessous :

En ce qui concerne les pistes de débardage, il a été expliqué précédemment que les gros arbres ne sont pas abattus, seules les tiges de moins de $20 \mathrm{~cm}$ de diamètre peuvent être détruites par le skidder en ouverture de piste. D'après les mesures de l'inventaire d'aménagement, ces tiges ne représentent que $9 \%$ de la biomasse forestière (en tonnes de carbone). L'ouverture d'une piste de débardage a donc une incidence modérée sur les stocks de carbone forestier. Cependant, cela demanderait à être confirmé par des mesures sur des placettes permanentes, en comparant les stocks de carbone mesurés avant et après l'ouverture d'une piste de débardage.

Le réseau de pistes de débardage a été relevé sur SIG pour différentes parcelles; il a ainsi été mesuré qu'il existe environ $85 \mathrm{~m} / \mathrm{ha}$ de pistes de débardage. Leur largeur est de $4 \mathrm{~m}$ (largeur du skidder) : l'emprise spatiale du réseau de débardage est donc de $340 \mathrm{~m}^{2} / \mathrm{ha}$.

Par ailleurs, la longueur maximale d'une piste de débardage est de $500 \mathrm{~m}$ (au-delà de cette distance, il est décidé de rallonger la route secondaire reliée à la piste de débardage). Afin de connaître la distance moyenne de débardage, un maillage théorique d'arbres a été créé sur SIG (un arbre exploitable tous les $100 \mathrm{~m}$ ), et la distance entre chacun de ces arbres et la route la plus proche a été calculée. Cette distance théorique "à vol d'oiseau " est en moyenne de $218 \mathrm{~m}$. La valeur retenue pour la suite des calculs 
est $250 \mathrm{~m}$ de longueur de piste de débardage, afin de prendre en compte les détours probables liés aux marécages et autres obstacles au passage du skidder.

Il n'existe pas de normes nationales sur la densité et la longueur des pistes de débardage. Les pratiques actuelles seront donc retenues pour le scénario de référence.

Finalement, les émissions liées à l'exploitation forestière dans le scénario de référence peuvent être estimées de la manière suivante :

$$
\mathrm{C}_{\mathrm{BSL}}=-\left(\mathrm{D}_{\mathrm{RI}, \mathrm{RII}} \times \mathrm{C}_{\mathrm{m}}+\mathrm{D}_{\mathrm{DEB}} \times \mathrm{C}_{\mathrm{m}} \times \mathrm{P}_{<20}\right)
$$

Où :

- $\mathrm{C}_{\mathrm{BSL}}=$ les émissions de carbone dans le scénario de référence $\left(\mathrm{tCO}_{2} / \mathrm{an}\right)$

- $\mathrm{C}_{\mathrm{m}}=$ le stock moyen de $\mathrm{CO}_{2}$ par hectare, sur les concessions du Volet 2

- $\mathrm{D}_{\mathrm{RI}, \mathrm{RII}}=$ la superficie de réseau routier primaire et secondaire ouverte par an (ha/an)

- $\mathrm{D}_{\mathrm{DEB}}=$ la superficie de réseau de débardage ouverte par an (ha/an)

- $\quad \mathrm{p}_{<20}=$ la proportion de biomasse affectée par l'ouverture des pistes de débardage (ici, uniquement les tiges inférieures à $20 \mathrm{~cm}$ de diamètre, c'est-à-dire $9 \%$ ).

\subsection{Scénario de projet : activités prévues, quantification}

Comme cela a été démontré plus haut, et au vu des observations réalisées pendant la mission de terrain, il a été retenu plusieurs leviers permettant de limiter la diminution des stocks de carbone liée à l'exploitation forestière :

- Réduction de la longueur des routes (par substitution par des pistes de débardage, donc avec un allongement de la distance de débardage) ;

- Réduction de la largeur des routes, en particulier des bandes d'ensoleillement ;

- Réduction de la proportion d'arbres coupés sur les bandes d'ensoleillement lors de la création de la route.

Afin d'estimer la réduction de la longueur des routes qui pourrait être atteinte grâce au scénario de projet, une analyse du réseau existant a été réalisée sur SIG. Tous les tronçons terminaux de route pouvant être substitués par des pistes de débardage d'une longueur maximale de $1500 \mathrm{~m}$ (pour atteindre les extrémités des blocs d'exploitation, donc les potentiels arbres exploitables situés aux confins des blocs) ont été supprimés et remplacés par des pistes de débardage (voir exemple du bloc d'exploitation $n^{\circ} 91$ sur la concession 28/03, Carte 7) pour simuler le scénario de projet. La diminution de la densité du réseau routier a ainsi pu être chiffrée (voir Tebleau 4).

Si la longueur maximale des pistes de débardage du projet est de $1500 \mathrm{~m}$, la distance moyenne « à vol d'oiseau " entre l'arbre exploitable et le parc situé en bord de route secondaire (distance calculée théoriquement selon la procédure expliquée en 1.2 Élaboration du scénario de référence $\mathrm{p}$.) est de $450 \mathrm{~m}$, contre $250 \mathrm{~m}$ dans le scénario de référence.

De plus, il a été possible d'estimer la réduction de la largeur des routes en se fondant sur les chiffres conseillés par le formateur EFIR de la SODEFOR, avec des emprises maximales totales de 22,0 m et de $16,0 \mathrm{~m}$ respectivement pour les routes principales et secondaires.

Cette modélisation permet de comparer la superficie affectée par les infrastructures routières entre les scénarii de référence et le scénario de projet (voir Tableau 4).

En plus de réduire la longueur et la largeur des routes principales et secondaires, il est également possible de réduire la proportion d'arbres abattus sur les bandes d'ensoleillement. En effet, actuellement, la quasi-intégralité de la végétation est détruite lors de la formation des bandes d'ensoleillement. La végétation se reconstitue rapidement ensuite, mais uniquement sur la base d'espèces pionnières à bois léger : le stock de carbone reste donc faible. En fait, d'après les mesures effectuées, le stock de carbone estimé sur les différentes placettes de bandes d'ensoleillement dépend fortement des quelques gros arbres qui ont été préservés lors des travaux d'ouverture. Finalement, plutôt que d'abattre systématiquement la quasiintégralité des arbres de part et d'autre de la route, le respect des normes EFIR devrait permettre de limiter la perte de carbone forestier; en effet, la création des bandes d'ensoleillement doit obéir à une règle unique selon laquelle la plateforme de la route doit recevoir le maximum de rayons solaires entre 11 heures et 15 heures. Il faut donc identifier et abattre la majorité des arbres qui projettent de l'ombre sur la route à ces heures-là. Ainsi, selon l'orientation de la route, la hauteur et la disposition des arbres, tous les arbres ne nécessitent pas d'être abattus pour améliorer l'ensoleillement de la route. 
Tableau 4 : Superficie affectée par les infrastructures routières selon les différents scénarii

\begin{tabular}{|c|c|c|c|c|c|c|}
\hline & & \multirow[t]{2}{*}{ Densité } & \multicolumn{3}{|c|}{ Largeur } & \multirow{2}{*}{$\begin{array}{l}\text { Surface } \\
\text { affectée }\end{array}$} \\
\hline & & & $\begin{array}{l}\text { Bande de } \\
\text { roulement }\end{array}$ & $\begin{array}{c}\text { Bande } \\
\text { d'ensoleillement }\end{array}$ & $\begin{array}{l}\text { Emprise } \\
\text { totale }\end{array}$ & \\
\hline \multirow[t]{3}{*}{$\begin{array}{l}\text { ROUTES } \\
\text { PRINCIPALES }\end{array}$} & $\begin{array}{l}\text { BASELINE (Pratiques } \\
\text { actuelles) }\end{array}$ & $5,9 \mathrm{~m} / \mathrm{ha}$ & $6,9 \mathrm{~m}$ & $16,2 \mathrm{~m}$ & $39,3 \mathrm{~m}$ & $229,9 \mathrm{~m}^{2} / \mathrm{ha}$ \\
\hline & $\begin{array}{l}\text { BASELINE (Normes } \\
\text { nationales) }\end{array}$ & $5,9 \mathrm{~m} / \mathrm{ha}$ & NA & NA & $30,0 \mathrm{~m}$ & $175,5 \mathrm{~m}^{2} / \mathrm{ha}$ \\
\hline & SCÉNARIO DE PROJET & $5,2 \mathrm{~m} / \mathrm{ha}$ & $7,0 \mathrm{~m}$ & $7,5 \mathrm{~m}$ & $22,0 \mathrm{~m}$ & $113,7 \mathrm{~m}^{2} / \mathrm{ha}$ \\
\hline \multirow[t]{3}{*}{$\begin{array}{l}\text { ROUTES } \\
\text { SECONDAIRES }\end{array}$} & $\begin{array}{l}\text { BASELINE (Pratiques } \\
\text { actuelles) }\end{array}$ & $6,0 \mathrm{~m} / \mathrm{ha}$ & $6,8 \mathrm{~m}$ & $9,0 \mathrm{~m}$ & $24,8 \mathrm{~m}$ & $148,3 \mathrm{~m}^{2} / \mathrm{ha}$ \\
\hline & $\begin{array}{l}\text { BASELINE (Normes } \\
\text { nationales) }\end{array}$ & $6,0 \mathrm{~m} / \mathrm{ha}$ & NA & NA & $30,0 \mathrm{~m}$ & $179,1 \mathrm{~m}^{2} / \mathrm{ha}$ \\
\hline & SCÉNARIO DE PROJET & $2,0 \mathrm{~m} / \mathrm{ha}$ & $6,0 \mathrm{~m}$ & $5,0 \mathrm{~m}$ & $16,0 \mathrm{~m}$ & $31,5 \mathrm{~m}^{2} / \mathrm{ha}$ \\
\hline \multirow[t]{3}{*}{$\begin{array}{l}\text { PISTES DE } \\
\text { DÉBARDAGE }\end{array}$} & $\begin{array}{l}\text { BASELINE (Pratiques } \\
\text { actuelles) }\end{array}$ & $85,0 \mathrm{~m} / \mathrm{ha}$ & $4,0 \mathrm{~m}$ & $0,0 \mathrm{~m}$ & $4,0 \mathrm{~m}$ & $340,0 \mathrm{~m}^{2} / \mathrm{ha}$ \\
\hline & $\begin{array}{l}\text { BASELINE (Normes } \\
\text { nationales) }\end{array}$ & $85,0 \mathrm{~m} / \mathrm{ha}$ & $4,0 \mathrm{~m}$ & $0,0 \mathrm{~m}$ & $4,0 \mathrm{~m}$ & $340,0 \mathrm{~m}^{2} / \mathrm{ha}$ \\
\hline & SCÉNARIO DE PROJET & $89,7 \mathrm{~m} / \mathrm{ha}$ & $4,0 \mathrm{~m}$ & $0,0 \mathrm{~m}$ & $4,0 \mathrm{~m}$ & $358,7 \mathrm{~m}^{2} / \mathrm{ha}$ \\
\hline
\end{tabular}

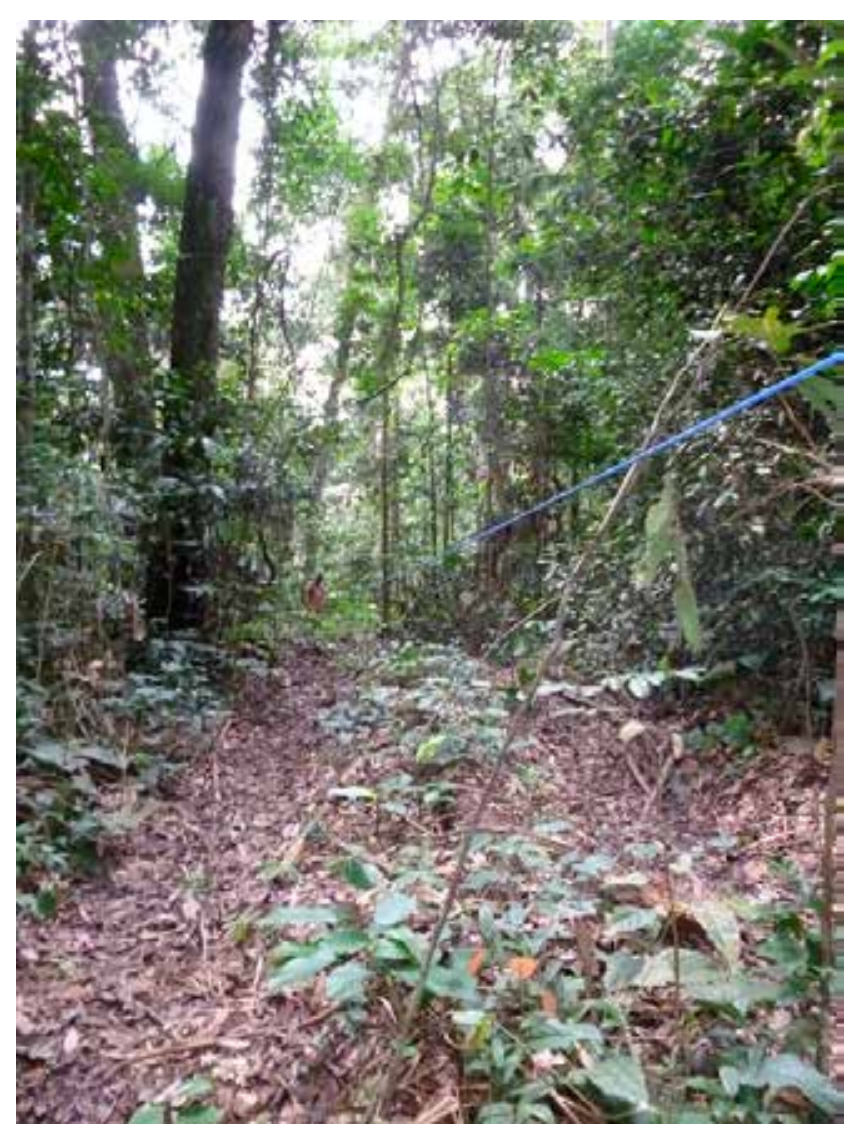

Piste de débardage de moins d'un an Source: FRM 2013

Les hypothèses fixées pour évaluer le bilan carbone du scénario de projet sont les suivantes :

- Le stock moyen de carbone forestier sur les concessions concernées par le Volet 2 correspond à $471 \mathrm{tCO}_{2} \mathrm{e} / \mathrm{ha}$;
- La biomasse forestière est constituée à $79 \%$ de tiges supérieures à $30 \mathrm{~cm}$ (d'après les données de l'inventaire d'aménagement) ;

- Les routes secondaires sont créées pour une utilisation courte (une année) tandis que les routes principales sont construites pour plusieurs années (en moyenne 4 ans) ;

- Un quart des arbres de plus de $30 \mathrm{~cm}$ situés dans l'emprise des bandes d'ensoleillement peuvent être préservés ;

- 22500 ha de forêt sont exploités chaque année sur l'ensemble des cinq concessions couvertes par le Volet 2 (soit 1/25 de la superficie utile totale $\left.{ }^{9}\right)$ avec une ouverture de routes correspondant aux surfaces indiquées dans le Tableau 4.

La diminution des émissions de carbone biogénique grâce au scénario de projet (et par rapport à un scénario de référence fondé sur les normes nationales) est évaluée dans le Tableau 5, en se fondant sur le calcul suivant :

$$
\begin{aligned}
& \mathrm{C}_{\mathrm{PJT}}=-\left(\mathrm{D}_{\mathrm{BR}(\mathrm{RI}, \mathrm{RII})} \times \mathrm{C}_{\mathrm{m}}+\mathrm{D}_{\mathrm{BE}(\mathrm{RI}, \mathrm{RII})} \times \mathrm{C}_{\mathrm{m}} \times \mathrm{p}_{<30}\right. \\
& \left.\mathrm{D}_{\mathrm{BE}(\mathrm{RI}, \mathrm{RII})} \times \mathrm{C}_{\mathrm{m}} \times \mathrm{p}_{>30} \times \mathrm{p}_{\mathrm{PRV}}+\mathrm{D}_{\mathrm{DEB}} \times \mathrm{C}_{\mathrm{m}} \times \mathrm{p}_{<20}\right)
\end{aligned}
$$

Où :

- $\quad \mathrm{C}_{\mathrm{PJT}}=$ les émissions de carbone dans le scénario de projet $\left(\mathrm{tCO}_{2} / \mathrm{an}\right)$

- $\mathrm{C}_{\mathrm{m}}=$ le stock moyen de $\mathrm{CO}_{2}$ par hectare, sur les concessions du Volet 2

9 La règlementation fixe une durée de rotation minimale de 25 ans. 
- $\quad \mathrm{D}_{\mathrm{BR}(\mathrm{RI}, \mathrm{RII})}=$ la superficie des bandes de roulement du réseau routier primaire et secondaire ouvertes par an (ha/an)

- $\quad \mathrm{D}_{\mathrm{BE}(\mathrm{RI}, \mathrm{RII})}=$ la superficie des bandes d'ensoleillement du réseau routier primaire et secondaire ouvertes par an (ha/an)

- $\mathrm{P}_{<30}=$ la proportion de biomasse dont les tiges sont inférieures à $30 \mathrm{~cm}$ de diamètre et qui seront intégralement détruites pour la formation des bandes d'ensoleillement, évaluée ici à $21 \%$

- $\mathrm{p}_{>30}=$ la proportion de biomasse dont les tiges sont supérieures à $30 \mathrm{~cm}$ de diamètre et qui seront partiellement détruites pour la formation des bandes d'ensoleillement, évaluée ici à $79 \%$

- $\mathrm{P}_{\mathrm{PRV}}=$ la proportion de biomasse parmi les tiges supérieures à $30 \mathrm{~cm}$ de diamètre qui seront intégralement détruites pour la formation des bandes d'ensoleillement, évaluée ici à $75 \%$

- $\quad \mathrm{D}_{\mathrm{DEB}}=$ la superficie de réseau de débardage ouverte par an (ha/an)

Tableau 5 : Crédits carbone du Volet 2

\begin{tabular}{|c|c|c|c|}
\hline \multirow[b]{2}{*}{ Année } & \multicolumn{2}{|c|}{$\begin{array}{l}\text { Diminution du stock de } \\
\text { carbone forestier }\left(\mathrm{tCO}_{2}\right)\end{array}$} & \multirow[t]{2}{*}{ Gain $\mathrm{tCO}_{2}$} \\
\hline & Baseline & $\begin{array}{l}\text { Scénario de } \\
\text { projet }\end{array}$ & \\
\hline 1 & -309950 & -148950 & 160999 \\
\hline 2 & -309950 & -148950 & 160999 \\
\hline 3 & -309950 & -148950 & 160999 \\
\hline 4 & -309950 & -148950 & 160999 \\
\hline 5 & -309950 & -148950 & 160999 \\
\hline 6 & -309950 & -148950 & 160999 \\
\hline 7 & -309950 & -148950 & 160999 \\
\hline 8 & -309950 & -148950 & 160999 \\
\hline 9 & -309950 & -148950 & 160999 \\
\hline 10 & -309950 & -148950 & 160999 \\
\hline 11 & -309950 & -148950 & 160999 \\
\hline 12 & -309950 & -148950 & 160999 \\
\hline 13 & -309950 & -148950 & 160999 \\
\hline 14 & -309950 & -148950 & 160999 \\
\hline 15 & -309950 & -148950 & 160999 \\
\hline 16 & -309950 & -148950 & 160999 \\
\hline 17 & -309950 & -148950 & 160999 \\
\hline 18 & -309950 & -148950 & 160999 \\
\hline 19 & -309950 & -148950 & 160999 \\
\hline 20 & -309950 & -148950 & 160999 \\
\hline TOTAL & -6198997 & -2979009 & 3219988 \\
\hline
\end{tabular}

- $\mathrm{P}_{<20}=$ la proportion de biomasse impactée par l'ouverture des pistes de débardage (ici, uniquement les tiges inférieures à $20 \mathrm{~cm}$ de diamètre, c'est-à-dire $9 \%$ ).

Les émissions évitées annuellement se calculent par la différence entre $\mathrm{C}_{\mathrm{PJT}}$ et $\mathrm{C}_{\mathrm{BSL}}$.

Certains éléments n'ont pas été pris en compte dans les calculs, notamment la superficie affectée par les parcs à grumes. Ceux-ci ont en effet été considérés comme constants entre les deux scénarii : leur nombre et leur superficie ne devraient pas être modifiés par le scénario de projet.

Par ailleurs, les émissions de gaz à effet de serre directement liées au fonctionnement des véhicules n'ont pas non plus été prises en compte. Difficiles à estimer (les émissions dues aux grumiers devraient diminuer mais celles dues aux skidder devraient augmenter), elles pourraient faire l'objet d'une étude plus approfondie.

En 20 ans (durée du projet REDD+ de la Lukénie), près 3,6 millions de crédits carbone devraient être délivrés pour le Volet 2 ; un chiffre qui atteindrait 3,2 millions si c'est le scénario de référence fondé sur les pratiques actuelles qui est choisi.

\subsection{Système de suivi}

Un protocole de mesure de biomasse, identique à celui présenté ci-dessus, pourra être réalisé sur les bandes d'ensoleillement ; d'après les mesures déjà accomplies et la grande hétérogénéité observée, 156 transects devront être effectués à intervalles réguliers (tous les 5 ans) dans les différents types de peuplement forestier. Ces mesures permettront d'évaluer les stocks de carbone contenus sur les bandes d'ensoleillement et ainsi vérifier les estimations ex-ante, dans un intervalle de confiance de $95 \%$ et avec une erreur relative de $10 \%$. Des mesures identiques devront également être réalisées aux abords de routes " témoins " où les normes EFIR ne sont pas encore appliquées, donc en dehors de la zone de projet. Pour cela, la concession SODEFOR la plus proche (concession 21/03, voisine de la concession 28/03 mais située en dehors de la zone du projet pilote REDD+ de la Lukénie) pourra être étudiée.

Un protocole de mesure de biomasse devra également être mis en place au niveau des pistes de débardage. À cette fin, des transects de mesures seront réalisés 
peu de temps avant l'ouverture du peuplement, la planification de l'exploitation permettant de connaître avec une certaine précision l'emplacement des futures pistes de débardage. Des mesures identiques devront être de nouveau réalisées sur les mêmes placettes, peu de temps après le passage du tracteur de débardage, afin de connaître réellement l'incidence du débardage sur la végétation. Ces données permettront d'affiner le modèle de suivi des stocks de carbone. Ces placettes permanentes devront être suivies régulièrement dans le temps afin d'avoir des données sur la régénération naturelle sur piste de débardage.

En plus des mesures de la largeur des infrastructures et du stock de carbone associé, un suivi de la longueur des routes et pistes de débardage devra également être réalisé. Cela pourrait être fait grâce à l'analyse d'images satellite. Ainsi, une image SPOT de la zone d'étude datant de janvier 2013 a été acquise, en partenariat avec l'IRD. Des méthodes de traitement d'images seront appliquées afin de détecter le réseau de pistes de débardage grâce à ces images uniquement. En parallèle, des relevés GPS des pistes de débardage de la même zone ont été réalisés en janvier 2013 afin de vérifier la pertinence des résultats issus de la télédétection. Si la télédétection des pistes de débardage s'avère fiable, cette méthodologie pourra être utilisée dans le système MRV afin de suivre le linéaire de pistes de débardage au cours du temps.

\section{5 Éléments d'analyse financière}

Les différents éléments d'analyse financière qui suivent sont donnés à titre indicatif et s'appuient sur des hypothèses qui restent à consolider.

En augmentant la longueur de débardage, le temps consacré au débardage des bois augmentera, entraînant un coût supplémentaire pour le concessionnaire.

En effet, la distance moyenne de débardage avec le projet passera de $250 \mathrm{~m}$ à $450 \mathrm{~m}$, d'après les estimations réalisées plus haut. D'après l'analyse issue de J. Estève ${ }^{10}$, le temps nécessaire à une rotation de débardage passera donc de 12,3 min à 16,2 min. En effet, le temps de manipulation de la grume sur place, supposé indépendant de la distance de débardage, est évalué à 7,5 min; le temps de déplacement aller

10 Estève, 1968, «Débardage second par tracteurs articulés à pneus au Gabon ", Bois et forêts des tropiques, $n^{\circ} 117$, janvier-février 1968. et retour entre le parc à grumes et le bois à débarder augmente proportionnellement avec la distance de débardage (à raison de $52 \mathrm{~m}$ parcourus par minute, en moyenne). Finalement, à cause de l'augmentation $\mathrm{du}$ temps de rotation, la production horaire par machine passera donc de $14,1 \mathrm{t} / \mathrm{h}$ à $10,7 \mathrm{t} / \mathrm{h}$, c'està-dire que la mise en place du projet nécessitera une vingtaine de jours supplémentaires par an pour débarder tout le bois exploité. À 788 euros/jour le coût du débardage, et avec $15000 \mathrm{~m}^{3}$ de bois évacués chaque année, le surcoût d'exploitation entraîné par le Volet 2 serait de 18777 euros par an.

De même, la réduction de la largeur des bandes d'ensoleillement et du nombre d'arbres abattus sur ces bandes risque de rendre les routes impraticables certains jours supplémentaires par rapport au scénario de référence, pendant la saison des pluies, ce qui allongera d'autant le nombre de jours nécessaires au transport des grumes par camion jusqu'au beach. En supposant que la proportion de jours non travaillés pour cause d'imprévus soit de $15 \%$ dans le scénario de référence (soit 17 jours travaillés par mois sur 20 jours ouvrés) et passe à $30 \%$ dans le scénario de projet (soit 14 jours travaillés par mois), le nombre de mois de travail par grumier et par an passerait de 3,5 mois à 4,3 mois, soit un surcoût de 41867 euros par an.

En revanche, en remplaçant les routes secondaires par des pistes de débardage plus longues, le nombre de routes à ouvrir chaque année va diminuer, ce qui entraînera des économies pour l'entreprise. En effet, il a été démontré plus haut que la densité du réseau de routes secondaires est de $6 \mathrm{~m} /$ ha dans le scénario de référence; dans le scénario de projet, cette densité devrait passer à $2 \mathrm{~m} / \mathrm{ha}$. Avec 22500 ha exploités

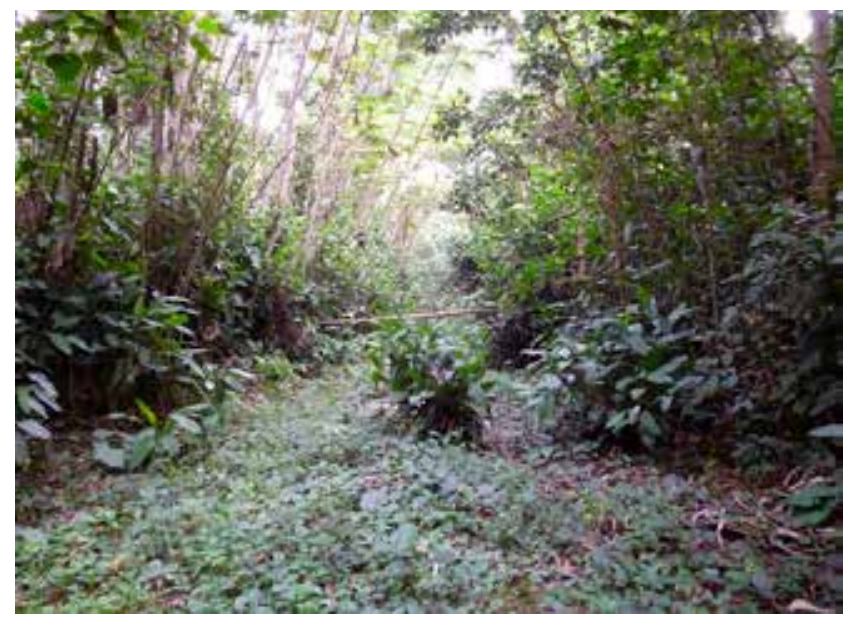

Route principale abandonnée depuis 6 ans Source: FRM 2013 
chaque année, le linéaire de routes secondaires à ouvrir devrait donc passer de $134 \mathrm{~km} / \mathrm{an}$ à $44 \mathrm{~km} /$ an. À 4000 euros $/ \mathrm{km}$ le coût d'ouverture d'une route forestière, les économies réalisées grâce à ce volet s'élèveraient donc 360000 euros par an. Les routes principales étant construites pour plusieurs années, et étant parfois placées sur d'anciennes routes d'exploitation (sachant que le coût de réouverture de route est moindre que le coût d'ouverture), elles n'ont pas été prises en compte dans ces estimations. Cette économie est non seulement financière, mais il s'agit également d'un gain de temps : la productivité en ouverture de route étant de $800 \mathrm{~m} /$ jour, près de 112 jours ne seraient plus consacrés à l'ouverture de routes grâce au scénario de projet.

Ces différents éléments permettent d'estimer les surcoûts et les économies directement liés au changement de méthodes d'exploitation. Mais une part importante des coûts de ce volet sera liée à la formation des techniciens à ces nouvelles pratiques ainsi qu'au personnel en charge de la mise en œuvre. D'autre part, une grande partie des coûts est directement liée au carbone : enregistrement du projet, validation de la méthodologie, monitoring et suivi MRV. Le budget total nécessaire à la mise en œuvre du Volet 2 s'élève à 7,7 millions d'euros en 20 ans, soit près de 386000 euros/an ; la Figure 10 indique la ventilation de ce budget dans les différentes rubriques. La gestion technique du projet paraît particulièrement importante, ce qui s'explique par les déplacements entre le siège (qui sera

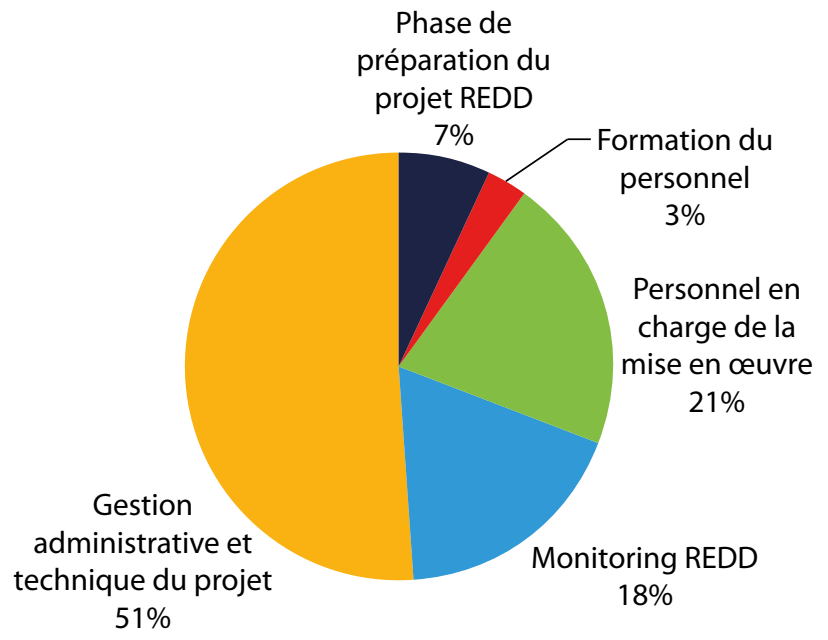

Figure 10 : Ventilation du budget total du Volet 2 dans les différentes rubriques

Source: FRM 2013

probablement situé à Nténo, sur la concession 28/03) et les activités de terrain, étant donné la grande superficie couverte parprotection ce volet et les fréquentes mesures sur le terrain qui seront nécessaires. Cependant, si cette rubrique paraît proportionnellement importante dans le Volet 2, elle est en valeur absolue moins élevée que le budget alloué à la même rubrique dans le Volet 1.

Le coût de revient de la tonne de $\mathrm{CO}_{2}$ s'élèverait ainsi à 2,0 euros $/ \mathrm{tCO}_{2} \mathrm{e}$, ce qui assurerait un prix tout à fait compétitif sur le marché du carbone. Mais il s'agit là d'une première évaluation qui demandera à être validée. 


\section{Volet 3 : mise en conservation}

\section{1 Élaboration du scénario de référence}

Les concessions $24 / 03$ et 29/03 sont en cours d'aménagement afin d'être exploitées durablement au cours des prochaines années. Cependant, s'il était finalement décidé de ne pas mettre en place d'exploitation forestière (contrairement à ce qui est actuellement prévu), les émissions liées au prélèvement de bois et à l'ouverture des routes d'exploitation seraient totalement inexistantes. Ainsi, le scénario de référence sera ici l'exploitation forestière obéissant aux normes nationales et aux pratiques conventionnelles en cours dans les concessions voisines, selon les données établies lors de l'inventaire d'aménagement ; tandis que le scénario de projet sera l'absence d'exploitation forestière et la mise en conservation volontaire.

La méthodologie de calculs REDD+ à suivre lors de la conversion d'une concession exploitée en une concession de conservation est la méthodologie VM0010. C'est la version 1.1 (novembre 2011) de cette méthodologie qui a été utilisée pour ce rapport, bien qu'elle soit en cours de révision et ne soit plus valide depuis septembre 2012; une nouvelle version est prévue pour avril 201311.

Le tableau suivant répertorie les émissions liées à l'exploitation forestière (scénario de référence) qui doivent être prises en compte d'après la méthodologie :

Il s'est avéré que les émissions liées à la décomposition du bois mort des arbres exploités et à la conversion des produits ligneux n'étaient pas véritablement prises en compte dans cette méthodologie ; c'est d'ailleurs pourquoi cette version est en cours de révision. Faute de méthodologie adaptée et de données de terrain, ces deux éléments ne seront pas pris en compte dans l'élaboration du scénario de référence dans ce Volet 3 du projet pilote.

11 La nouvelle version a été mise à disposition après la fin de la présente étude, c'est pourquoi elle n’a pas été prise en compte. Toutefois, les changements de stocks liés à l'ouverture de la desserte forestière ne semblent pas être pris en compte non plus dans la version 1.2 de la méthodologie VM0010.

\author{
Inclus dans la modélisation \\ Émissions liées à l'extraction de bois \\ Émissions liées à la conversion des produits ligneux \\ Changement de stock lié à la régénération naturelle \\ consécutive à l'exploitation \\ Décomposition du bois mort des arbres exploités

\begin{tabular}{l}
$\begin{array}{l}\text { Exclus de la modélisation (par mesure de } \\
\text { conservation) }\end{array}$ \\
\hline Décomposition des arbres accidentellement abattus \\
lors de l'abattage \\
Décomposition des arbres abattus lors de la création \\
des pistes de débardage \\
Décomposition des arbres abattus lors de la création \\
du réseau routier \\
Émission de combustibles fossiles lors de l'exploitation
\end{tabular}

Finalement, n'ont donc été considérées pour l'élaboration du scénario de référence que les émissions directement liées à l'extraction de bois (donc à la diminution du stock de carbone forestier) et à la régénération naturelle consécutive à l'exploitation (donc à une augmentation du stock de carbone forestier).

\subsection{1 Émissions liées à l'extraction de bois}

Par mesure de conservation, on a considéré que seules les essences du groupe 1 (c'est-à-dire les essences systématiquement exploitées par le concessionnaire) seraient exploitées sur les concessions $24 / 03$ et 29/03, avec un diamètre minimum d'exploitation de $60 \mathrm{~cm}$. Pour calculer le volume ainsi représenté, les données issues de l'inventaire d'aménagement (voir Carte 3) ont été utilisées et intégrées au logiciel Forest Carbon Print-Stock (C) élaboré par FRM (voir p. 18).

Ainsi, la biomasse aérienne constituée par les arbres du groupe 1 de diamètre supérieur à $60 \mathrm{~cm}$ sur les concessions 24/03 et 29/03 s'élève à 2,2 tC/ha soit $8 \mathrm{tCO}_{2} \mathrm{e} / \mathrm{ha}$ (voir Carte 8). Il reste donc à déterminer la superficie vouée à être exploitée sur ces deux concessions pour estimer le stock total de carbone qui serait retiré au cours du projet dans le scénario de référence. 


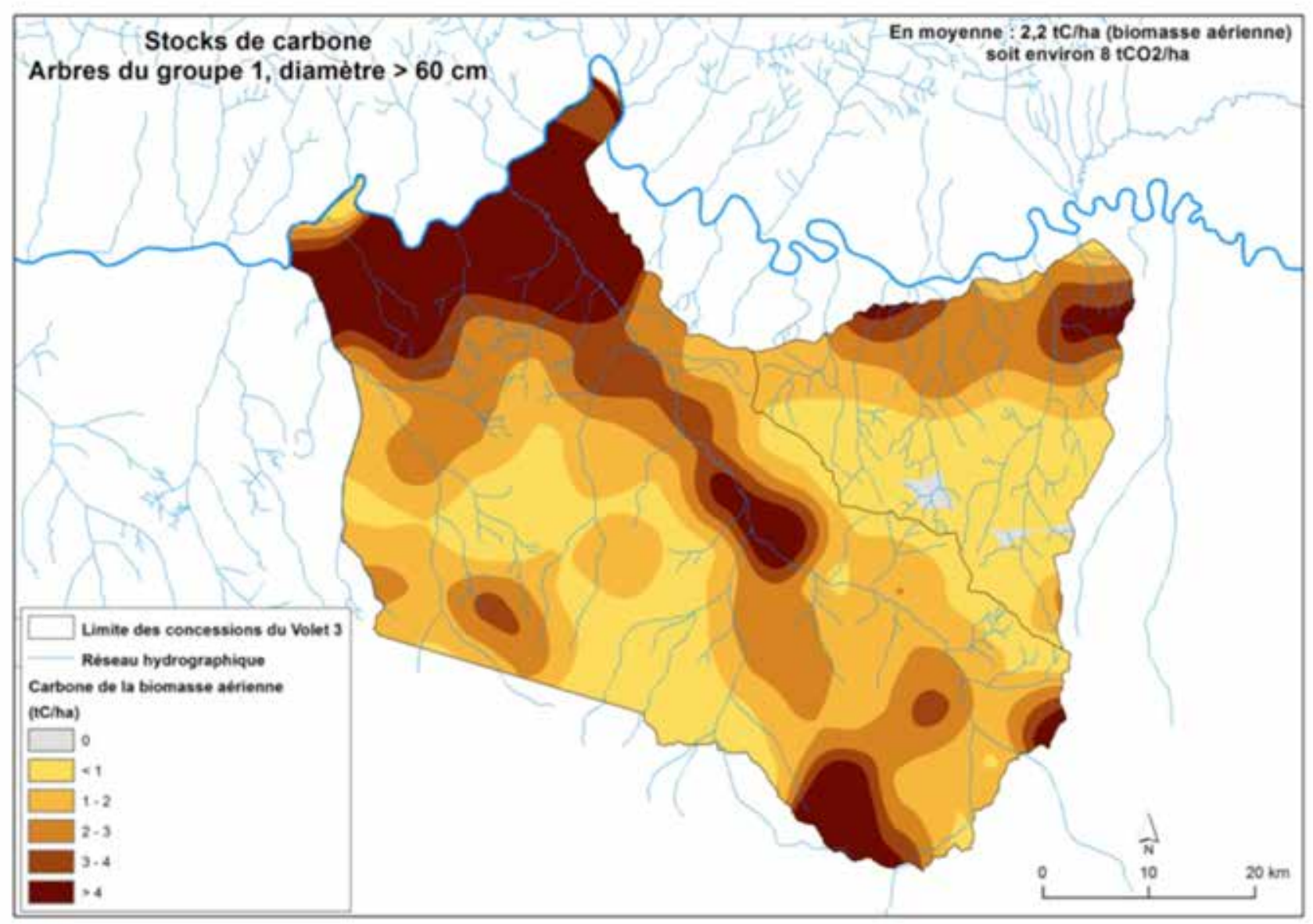

Carte 8 : Répartition des stocks de carbone contenus dans les arbres du groupe 1 supérieurs à $60 \mathrm{~cm}$ de diamètre Source: FRM 2013

La superficie vouée à être exploitée ne correspond pas à la "superficie utile", ou superficie productive, identifiée dans le rapport d'inventaire d'aménagement par télédétection. En effet, lors de l'élaboration du plan d'aménagement, la superficie totale de la concession est découpée en différentes séries d'aménagement (ou types d'affectation des terres) :

- Série de production (zone forestière vouée à être exploitée) ;

- Série de conservation (zone forestière qui ne sera pas exploitée) ;

- Série de protection (mise en place le long des cours d'eau et des zones fragiles) ;

- Zone à vocation agricole (vouée au développement des activités agricoles des populations locales).

Le plan d'aménagement étant encore en cours de réalisation, la série de production pour les concessions $24 / 03$ et 29/03 n'est pas encore définie. Sa superficie a donc été estimée approximativement, comme correspondant à $81 \%$ de la superficie totale (calcul réalisé à partir des autres concessions aménagées de la SODEFOR en République démocratique du Congo). La superficie de la série de production sur l'ensemble des deux concessions atteindrait donc environ 150000 ha. L'exploitation se faisant sur une rotation de 25 ans (temps de parcours de l'ensemble de la série de production avant retour sur les premières parcelles exploitées), 1/25 de cette superficie serait exploité chaque année en moyenne.

Finalement, on peut considérer dans le scénario de référence que $\mathbf{6} 000$ ha seraient exploités chaque année sur l'ensemble des deux concessions.

Enfin, les arbres du groupe 1 de diamètre supérieur à $60 \mathrm{~cm}$ et situés dans la série de production ne seront pas intégralement prélevés : certains arbres seront en effet préservés pour leur valeur culturelle (arbres sacrés pour les populations autochtones), d'autres pour servir de semenciers, d'autres enfin ne présenteront pas une qualité suffisante pour être valorisés. Les arbres du groupe 1 de diamètre supérieur à $60 \mathrm{~cm}$ représentent $8 \mathrm{tCO}_{2} /$ ha mais on 
peut considérer, par prudence, que seuls $60 \%$ de ces arbres seront réellement abattus.

Compte tenu de ces différents paramètres, on aboutit finalement à un stock prélevé de près de $5 \mathrm{tCO}_{2} /$ ha ou $\Delta \mathrm{C}=-29040 \mathrm{tCO}_{2} \mathrm{e} / \mathrm{an}$ dans le scénario de référence, en considérant une exploitation ciblée sur uniquement $60 \%$ du stock disponible constitué par les arbres du groupe 1 de diamètre supérieur à $60 \mathrm{~cm}$, d'après les résultats de l'inventaire d'aménagement, sur une série de production estimée à 6000 ha.

\subsubsection{Changement de stock lié à la régénération naturelle}

L'exploitation des arbres de gros diamètre provoque la formation de trouées ponctuelles dans le couvert forestier ; cet apport de lumière dynamise la régénération naturelle et induit donc une augmentation ponctuelle du stock de carbone. Par conséquent, cette régénération doit être prise en compte et être modélisée afin d'affiner les calculs de changements de stocks de carbone du scénario de référence.

La méthodologie VM0010 conseille d'estimer la séquestration de carbone liée à la régénération consécutive à l'exploitation comme suit :

$$
\Delta \mathrm{C}_{\mathrm{RG}}=\mathrm{A} \times(R g \times N a)
$$

Où $\Delta \mathrm{C}$ représente la séquestration de carbone liée à la régénération naturelle, en tonnes de carbone, $A$ la surface concernée, $R g$ le taux de régénération naturelle post-exploitation, en tC/ha/an et $\mathrm{Na}$ le nombre d'années depuis l'exploitation.

Le taux de régénération naturelle post-exploitation peut être estimé selon les méthodes suivantes :

- Données issues de mesures sur le terrain au cours du temps, dans la zone de référence, selon un protocole scientifique rigoureusement établi ;

- Données publiées concernant la régénération naturelle après exploitation de bois d'œuvre, dans une forêt similaire et dans une zone proche du projet ;

- Valeurs par défaut du GIEC sur la croissance de la biomasse aérienne en forêt naturelle.

Faute de données fiables spécifiques à la zone d'étude, ce sont donc les valeurs par défaut proposées par le GIEC qui ont été étudiées. En forêt tropicale humide mature, le GIEC préconise ainsi d'utiliser une valeur de croissance de la biomasse aérienne de $3,1 \mathrm{tC} / \mathrm{ha} / \mathrm{an}$.
Il reste donc à déterminer la superficie affectée par l'abattage, c'est-à-dire la superficie des trouées d'abattage. D'après Durrieu de Madron (2000)12, la superficie affectée par les dégâts d'abattage et de débardage peut être estimée comme suit :

$$
S=S_{E X} \times\left(1-\left(1 /(1+0,186 N)^{0,465}\right)\right)
$$

Où :

- $\quad S=$ la superficie des dégâts d'abattage au sol ;

- $S_{E X}=$ la superficie exploitée chaque année ;

- $\quad N N=$ le nombre de tiges abattues par hectare.

Si $S_{E X} S_{E X}=6000$ ha et $N N=0,7$ tige/ha, on obtient $S S=332$ ha. Cette superficie est constituée par la surface affectée par les pistes de débardage et les places d'abattage. Or, la superficie atteinte par les pistes de débardage a été estimée dans le cadre du Volet 2 et s'élève ici à 204 ha/an. Il devient dès lors possible de déterminer la superficie affectée par les places d'abattage, qui s'élève à 128 ha par an. La régénération dans les trouées d'abattage correspondrait donc à $1454 \mathrm{tCO}_{2} / \mathrm{an}$.

Finalement, en considérant le changement de stock lié au prélèvement de bois et celui lié à la régénération naturelle, l'évolution totale du stock dans le scénario de référence s'élèverait à :

$$
\Delta \mathrm{C} \Delta \mathrm{C}=-27586 \mathrm{tCO}_{2} / \mathrm{an} .
$$

Mais en suivant cette méthodologie, un élément important de l'exploitation forestière est omis : il s'agit de la construction du réseau routier d'évacuation des bois. Les arbres abattus pour permettre l'ouverture de cette desserte forestière induisent une diminution notable du stock de carbone forestier.

\subsubsection{Changement de stock lié à l'ouverture du réseau d'évacuation des bois}

Lors de la construction des routes principales, des routes d'ensoleillement et de leurs bandes d'ensoleillement, l'intégralité de la végétation est abattue, provoquant une diminution importante $\mathrm{du}$ stock de carbone forestier. Celui-ci est également affecté par les dégâts des pistes de débardage. Ces différents éléments ont été calculés lors de l'élaboration du scénario de référence du Volet 2, en fonction de la superficie de l'assiette annuelle de coupe et en se fondant sur une exploitation

12 Durrieu de Madron et al. (2000), Dégâts d'exploitation et de débardage en fonction de l'intensité d'exploitation en forêt dense humide d'Afrique centrale, Bois et forêts des tropiques, $\mathrm{n}^{\circ} 264$. 


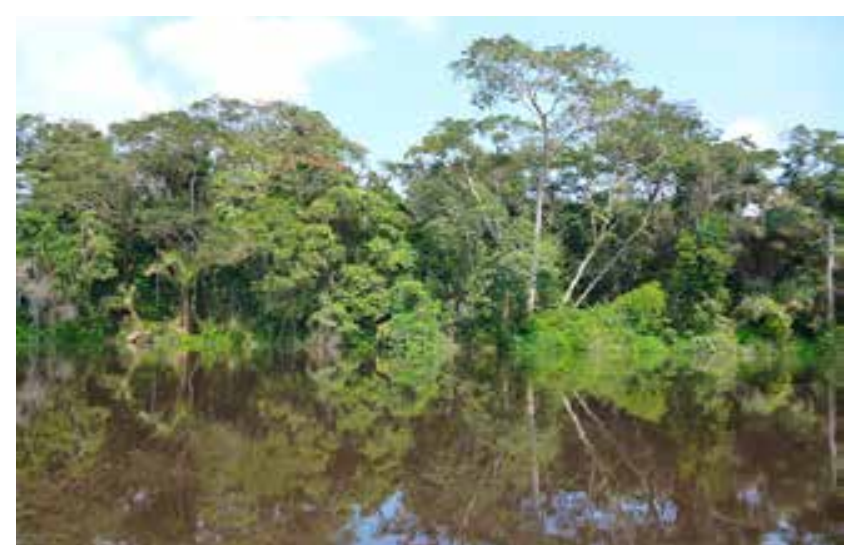

Rives de la Lukénie

Source: FRM 2013

conventionnelle répondant aux normes nationales; les calculs effectués dans le scénario de référence du Volet 2 pour évaluer l'incidence de l'ouverture du réseau routier sur le stock de carbone peuvent être repris ici.

Si la croissance de la végétation est considérée comme nulle sur la bande de roulement (sols trop tassés), elle n'est pas négligeable sur les bandes d'ensoleillement des routes et sur les pistes de débardage. Lévolution du stock de carbone liée à la régénération naturelle post-exploitation peut donc également être calculée, en prenant la même base de $3,1 \mathrm{tC} / \mathrm{ha} / \mathrm{an}$.

En rajoutant ces deux éléments dans les calculs (ouverture du réseau routier et régénération naturelle sur ces superficies), l'évolution du stock de carbone dans le scénario de référence s'élèverait à :

$$
\Delta \mathrm{C}=-109894 \mathrm{tCO}_{2} / \mathrm{an} .
$$

Finalement, le scénario de référence du Volet 3 est constitué de plusieurs composantes :

- (1) Diminution du stock de carbone forestier liée au retrait ponctuel de bois d'œuvre ;

- (2) Diminution du stock de carbone forestier liée à la destruction forestière pour la création des routes d'exploitation ;
- (3) Augmentation du stock de carbone forestier liée à la régénération post-exploitation dans les trouées d'abattage ;

- (4) Augmentation du stock de carbone forestier liée à la régénération post-exploitation sur les bandes d'ensoleillement des routes et les pistes de débardage.

Les composantes (2) et (4) ont des répercussions notables sur le stock de carbone forestier mais ne sont pas prises en compte dans la méthodologie VM0010.

Lorsque ce projet sera enregistré, il faudra donc développer et enregistrer une nouvelle version de cette méthodologie s'il est décidé de prendre en compte ces deux composantes.

Le Tableau 6 indique l'impact carbone de ces différentes composantes en tonnes de $\mathrm{CO}_{2}$ équivalent, en prenant pour référence une exploitation forestière conventionnelle qui répond aux normes nationales, avec une superficie de 6000 ha exploitée chaque année.

\subsection{Scénario de projet : activités prévues, quantification}

Le projet consiste ici à mettre en conservation volontaire les concessions 24/03 et 29/03. Aucune exploitation forestière n'aura lieu. Aucun arbre ne sera prélevé. Aucun réseau routier d'évacuation des bois ne sera donc construit non plus. En outre, l'hypothèse est faite que la forêt est en équilibre, le stock de carbone du scénario de projet reste donc le même sur toute la durée du projet.

Chaque année, le différentiel de stock de carbone entre le scénario de projet sera donc de

$$
\Delta \mathrm{C}=-109894 \mathrm{tCO}_{2} / \mathrm{an} .
$$

Par mesure conservative, les émissions de gaz à effet de serre directement liées au fonctionnement des véhicules d'exploitation dans le scénario de référence

Tableau 6 : Incidence de l'exploitation forestière conventionnelle sur les stocks de carbone (tCO 2 e). Scénario de

\begin{tabular}{|c|c|c|c|c|}
\hline \multicolumn{5}{|c|}{ Baseline : Incidence sur les stocks de carbone forestier $\left(\mathrm{tCO}_{2} \mathrm{e}\right)$} \\
\hline Prélèvement de bois & Réseau routier & Régénération dans trouées & Régénération sur le réseau routier & Total \\
\hline-29040 & -86175 & 1454 & 3867 & -109894 \\
\hline $26,4 \%$ & $78,4 \%$ & $-1,3 \%$ & $-3,5 \%$ & $100,0 \%$ \\
\hline
\end{tabular}
référence du Volet 3 
(grumiers, skidder, etc.), qui sont inexistantes dans le scénario de projet, ont été omises.

La diminution des émissions de carbone biogénique grâce au scénario de projet (et par rapport à un scénario de référence fondé sur les normes nationales) peut être estimée comme suit (Tableau 7) :

Par ailleurs, dans la mesure où l'exploitation forestière de la SODEFOR sur les concessions voisines obéit à un plan d'aménagement prônant une gestion durable des ressources, le prélèvement de bois sur ces concessions n'augmentera pas avec l'arrêt de l'exploitation sur les concessions $24 / 03$ et $29 / 03$. On peut donc considérer que les fuites (leakages) sont négligeables dans ce Volet 3.

\subsection{Système de suivi}

Des équipes réaliseront une mission par an pour vérifier l'absence d'exploitation illégale. Ces vérifications se feront aux alentours des voies

Tableau 7 : Crédits carbone du Volet 3

\begin{tabular}{|c|c|c|c|}
\hline \multirow[b]{2}{*}{ Année } & \multicolumn{2}{|c|}{$\begin{array}{l}\text { Diminution du stock de } \\
\text { carbone forestier }\left(\mathrm{tCO}_{2}\right)\end{array}$} & \multirow[b]{2}{*}{$\begin{array}{c}\text { Gain } \mathrm{tCO}_{2} \\
\text { annuel }\end{array}$} \\
\hline & Baseline & $\begin{array}{l}\text { Scénario de } \\
\text { projet }\end{array}$ & \\
\hline 1 & -109894 & 0 & 109894 \\
\hline 2 & -109894 & 0 & 109894 \\
\hline 3 & -109894 & 0 & 109894 \\
\hline 4 & -109894 & 0 & 109894 \\
\hline 5 & -109894 & 0 & 109894 \\
\hline 6 & -109894 & 0 & 109894 \\
\hline 7 & -109894 & 0 & 109894 \\
\hline 8 & -109894 & 0 & 109894 \\
\hline 9 & -109894 & 0 & 109894 \\
\hline 10 & -109894 & 0 & 109894 \\
\hline 11 & -109894 & 0 & 109894 \\
\hline 12 & -109894 & 0 & 109894 \\
\hline 13 & -109894 & 0 & 109894 \\
\hline 14 & -109894 & 0 & 109894 \\
\hline 15 & -109894 & 0 & 109894 \\
\hline 16 & -109894 & 0 & 109894 \\
\hline 17 & -109894 & 0 & 109894 \\
\hline 18 & -109894 & 0 & 109894 \\
\hline 19 & -109894 & 0 & 109894 \\
\hline 20 & -109894 & 0 & 109894 \\
\hline TOTAL & -2197885 & 0 & 2197885 \\
\hline
\end{tabular}

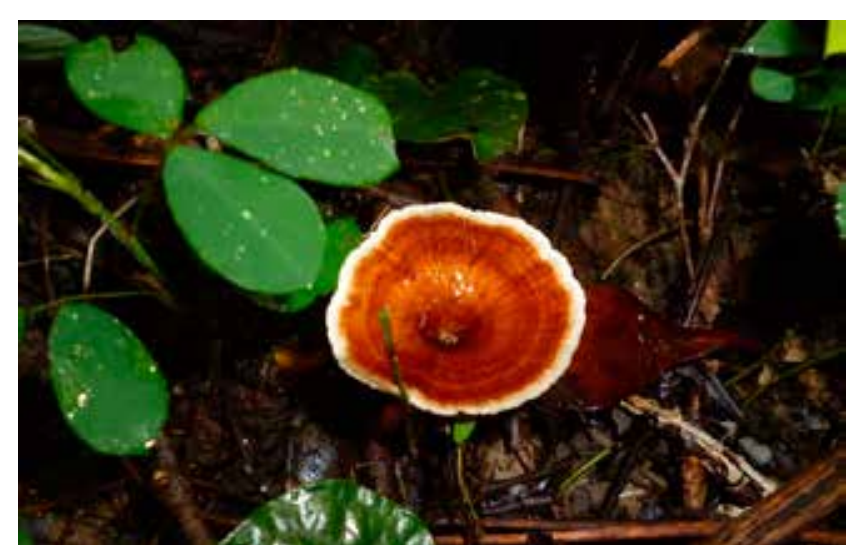

Mycète forestier Source: FRM 2013

d'accès (voie navigable constituée par la Lukénie au nord, proximité des villages et des quelques routes permanentes à l'est). En effet, toute exploitation forestière se produisant loin de toute desserte pourra être considérée comme étant négligeable et donc omise dans le calcul du scénario de projet.

Un suivi par télédétection tous les trois ans sera également mis en place pour vérifier l'absence de déforestation liée à l'exploitation (construction de desserte forestière, etc.).

Enfin, quelques placettes permanentes seront également mises en place dans les concessions voisines en exploitation, sur les pistes de débardage et les bandes d'ensoleillement, pour mesurer la dynamique de régénération et ainsi calculer plus précisément l'évolution du stock de carbone du scénario de référence. Ces placettes permanentes feront l'objet de mesures tous les trois ans : les opérateurs devront noter le diamètre et l'essence de tous les ligneux vivants de plus de $10 \mathrm{~cm}$ de diamètre présents sur la placette, afin d'évaluer l'évolution du stock de carbone sur ces placettes dans le temps et, par suite, un taux de croissance de la végétation sur ces placettes dégradées, en différenciant celles situées sur les bandes d'ensoleillement et celles situées sur les pistes de débardage. Les bandes d'ensoleillement bénéficiant d'une luminosité plus importante et d'un moindre tassement des sols que les pistes de débardage, il est probable que la dynamique de régénération soit plus grande sur ces bandes que sur les pistes de débardage.

\section{4 Éléments d'analyse financière}

Arrêter l'exploitation sur les concessions 24/03 et 29/03 provoquera une perte de bénéfice pour le concessionnaire. Évaluer cette perte de bénéfice 
permet de préciser le coût de mise en œuvre du projet, puisqu'elle vient s'ajouter aux coûts directs de mise en œuvre du projet.

D'après l'inventaire d'aménagement, le volume de bois exploitable parmi les arbres du groupe 1 (groupe rassemblant les essences systématiquement exploitées par le concessionnaire) s'élève à $1,69 \mathrm{~m}^{3} /$ ha dans la concession $24 / 03$ et à $2,15 \mathrm{~m}^{3} /$ ha dans la concession 29/03 (ce volume correspond au volume net constitué par les bois de diamètre supérieur ou égal au diamètre utile).

Lors de l'usinage et de la transformation, environ un tiers du volume de la grume peut être transformé en débités (rendement de transformation de $33 \%$ ). La marge nette obtenue à Kinshasa a été évaluée à 50 euros par $\mathrm{m}^{3}$ de planches issues d'essences nobles ${ }^{13}$ (cette marge prend en compte tous les coûts liés à l'exploitation). En considérant que $60 \%$ des arbres exploitables précédemment inventoriés seront prélevés, soit près de $8000 \mathrm{~m}^{3}$ par an, on arrive finalement à une production de plus de $2500 \mathrm{~m}^{3}$ de bois débité par an, soit un bénéfice de 130000 euros par an dans le scénario de référence, dans le cas d'une exploitation conventionnelle, pour les concessions $24 / 03$ et 29/03.

L'arrête de l'exploitation provoquerait donc une perte de près de 130000 euros/an pour le concessionnaire, soit près de 2,6 millions d'euros sur la durée du projet.

Une part importante des coûts de mise en œuvre de ce volet sera liée au personnel en charge de la mise en œuvre, notamment pour la surveillance et le suivi. De plus, une grande partie des coûts est directement liée au projet carbone lui-même : enregistrement du projet, validation de la méthodologie, monitoring et suivi MRV. La Figure 11 décrit la répartition du budget total du Volet 3 dans les différentes rubriques.

Enfin, une incertitude persiste au sujet de la taxe de superficie. Celle-ci s'élève à 0,5 dollar/ha/ an sur l'intégralité de la superficie utile, ce qui correspond donc à d'importantes sommes à reverser chaque année à l'État (plus de 71000 euros par an, soit 1,4 million d'euros en 20 ans pour les

13 Il s'agit d'une hypothèse de travail établie pour la rédaction de ce rapport ; les marges réelles sont variables selon les essences, les marchés, les performances industrielles, et constituent des données confidentielles qui ne nous ont pas été communiquées par SODEFOR.

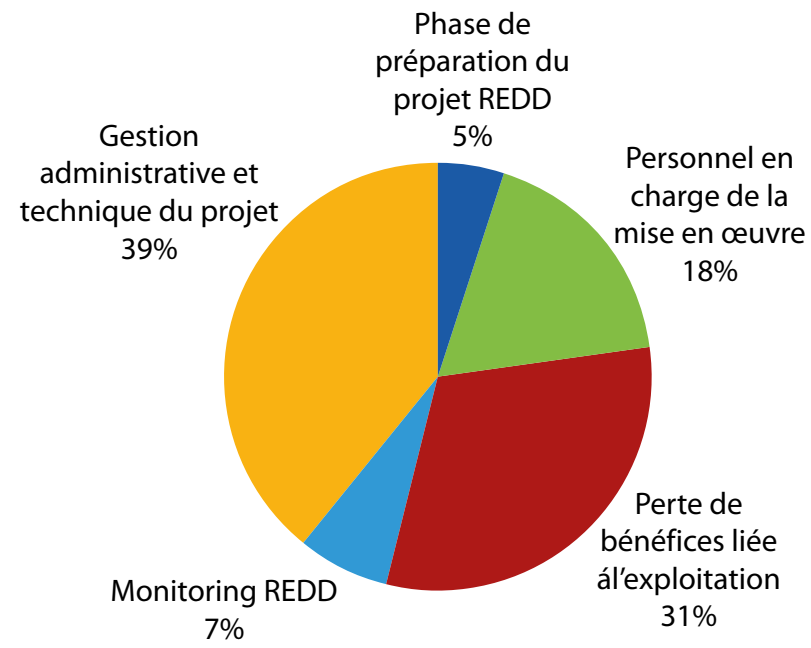

Figure 11 : Ventilation du budget total du Volet 3 dans les différentes rubriques

Source: FRM 2013

deux concessions concernées par le Volet 3). Il est possible que cette taxe soit annulée si les concessions sont converties en concessions de conservation, mais cela reste à confirmer. Dans tous les cas, cette conversion ne doit pas entraîner de perte de bénéfices pour l'État (aucune des parties prenantes du projet REDD ne doit ressortir perdante de l'exécution de ce projet, afin d'assurer le succès, la viabilité et la reproductibilité de ce dernier). Que ce soit sous la forme de taxe ou de crédits carbone, un montant au moins équivalent à cette somme devra donc être reversé à l'État.

De la même manière, lorsqu'une société exploite une concession forestière, elle doit verser une compensation financière aux populations riveraines dans le cadre des clauses sociales, par le biais d'un fonds de développement local (un fonds géré par un comité de représentants villageois qui sert à financer des infrastructures et des projets d'intérêt général : écoles, infrastructures de santé, routes de désenclavement, etc.). Le montant de cette compensation s'élève réglementairement entre 2 et 5 dollars $/ \mathrm{m}^{3}$ de bois commercialisé. Il a été envisagé un montant moyen de 4 dollars $/ \mathrm{m}^{3}$, ce qui représenterait environ 22900 euros/an, soit environ 460000 euros en 20 ans pour les deux concessions concernées par le Volet 3. Dans le scénario de projet, le bois de ces concessions ne sera certes pas exploité, mais les populations locales ne doivent pas pour autant subir de préjudice. Un montant au moins équivalent à la somme qu'elles auraient dû percevoir dans le scénario de référence devra donc leur être reversé. 
Le budget total du Volet 3 s'élève ainsi à 9,5 millions d'euros en 20 ans, soit en moyenne 475000 euros/ an. Ce qui aboutit à un coût de revient de la tonne de carbone de 4,3 euros $/ \mathrm{tCO}_{2}$. Le Volet 3 est donc celui qui présente un coût de la tonne de carbone le plus élevé (par rapport aux deux autres volets). Cela s'explique à la fois par le coût élevé du volet (à cause de la perte de bénéfices suite à l'arrêt de l'exploitation, notamment) et par les évitements d'émissions relativement faibles (faible production de crédits carbone liée à la faible superficie mise en conservation par rapport aux superficies concernées par les deux autres volets). 


\section{Bilan et perspectives}

\subsection{Bilan}

Le projet pilote REDD+ de la Lukénie est donc constitué de trois volets, synthétisés dans le tableau ci-dessous (Tableau 8).

Le Volet 1 semble donc être celui qui délivrerait le plus de crédits carbone ; les surfaces défrichées chaque année par les populations locales dans le scénario de référence étant en effet très importantes, la mise en place d'activités visant à limiter ces défrichements permet finalement de préserver des superficies importantes de forêts et, par suite, de générer des quantités importantes de crédits carbone.

Le Volet 3 présente quant à lui un coût de la tonne de $\mathrm{CO}_{2}$ relativement élevé par rapport aux deux autres volets. Cela s'explique par :

- la perte de bénéfices liée à l'arrêt des activités forestières et de la vente de bois, cet élément ayant été intégré à l'estimation du coût du projet ;

- la production de crédits carbone relativement modeste par rapport aux deux autres volets (ce qui est essentiellement dû à la superficie, moins importante pour ce volet que pour les deux autres).
L'analyse démontre que le prix de revient de la tonne de $\mathrm{CO}_{2}$ dans un projet de " conservation » dépend fortement de la rentabilité de l'exploitation qui serait enregistrée en l'absence de projet. Ce type de projet ne pourra être rentable que si la rentabilité de l'exploitation est faible, en raison de coûts de transport élevés ou d'une faible densité de ressource valorisable.

Par ailleurs, le Volet 2 paraît produire relativement peu de crédits carbone par rapport aux Volets 1 et 3 $\left(3,7 \mathrm{tCO}_{2} / \mathrm{ha}\right.$, contre $7,2 \mathrm{tCO} /$ ha en moyenne dans les deux autres volets), mais cela est compensé par la superficie importante concernée par le Volet 2.

Enfin, les crédits carbone délivrés par les Volets 2 et 3 seront constants au cours du temps (puisqu'ils dépendent de la superficie forestière exploitée chaque année dans le scénario de référence, une superficie globalement constante au cours du temps), alors que les crédits carbone délivrés par le Volet 1, en faibles quantités au début, augmenteront progressivement au cours du temps avec la montée en puissance du projet (diffusion des nouvelles pratiques auprès d'une part de plus en plus importante de la population). Cela est repris dans le graphique ci-dessous (Figure 12).

Tableau 8 : Bilan des trois volets du projet pilote REDD+ de la Lukénie ${ }^{a}$

\begin{tabular}{|c|c|c|c|c|}
\hline Volet & $\begin{array}{l}\text { Volet } 1 \text { : } \\
\text { Déforestation évitée }\end{array}$ & $\begin{array}{c}\text { Volet } 2 \text { : Exploitation } \\
\text { forestière à } \\
\text { impact réduit }\end{array}$ & $\begin{array}{c}\text { Volet } 3 \text { : Mise } \\
\text { en conservation } \\
\text { volontaire }\end{array}$ & TOTAL \\
\hline $\begin{array}{l}\text { Superficie totale } \\
\text { des concessions } \\
\text { concernées }\end{array}$ & 472790 ha & 967114 ha & 323287 ha & 1290401 ha \\
\hline \multirow{2}{*}{$\begin{array}{l}\text { Crédits carbone } \\
\text { attendus en } 20 \text { ans }\end{array}$} & $3547080 \mathrm{tCO}_{2}$ & $3584755 \mathrm{tCO}_{2}$ & $2197885 \mathrm{tCO}_{2}$ & $9329720 \mathrm{tCO}_{2}$ \\
\hline & $7,5 \mathrm{tCO}_{2} / \mathrm{ha}$ & $3,7 \mathrm{tCO}_{2} / \mathrm{ha}$ & $6,8 \mathrm{tCO}_{2} / \mathrm{ha}$ & $7,2 \mathrm{tCO}_{2} / \mathrm{ha}$ \\
\hline $\begin{array}{l}\text { Coût de la mise en } \\
\text { oeuvre du projet } \\
\text { sur } 20 \text { ans }\end{array}$ & $13299137 € 7$ & $287716 €$ & $9500681 €$ & $30087534 €$ \\
\hline Coût à I'hectare & $28 € /$ ha & $8 € /$ ha & $29 € /$ ha & $23 € /$ ha \\
\hline $\begin{array}{l}\text { Coût de revient de la } \\
\text { tonne de } \mathrm{CO}_{2}\end{array}$ & $3,7 € / \mathrm{tCO}_{2}$ & $2,0 € / \mathrm{tCO}_{2}$ & $4,3 € / \mathrm{tCO}_{2}$ & $3,2 € / \mathrm{tCO}_{2}$ \\
\hline
\end{tabular}

a Rappel : les superficies relatives aux volets 1 et 2 se superposent partiellement, ce qui explique que la superficie totale du projet REDD+ est inférieure à la somme des superficies des trois volets. 


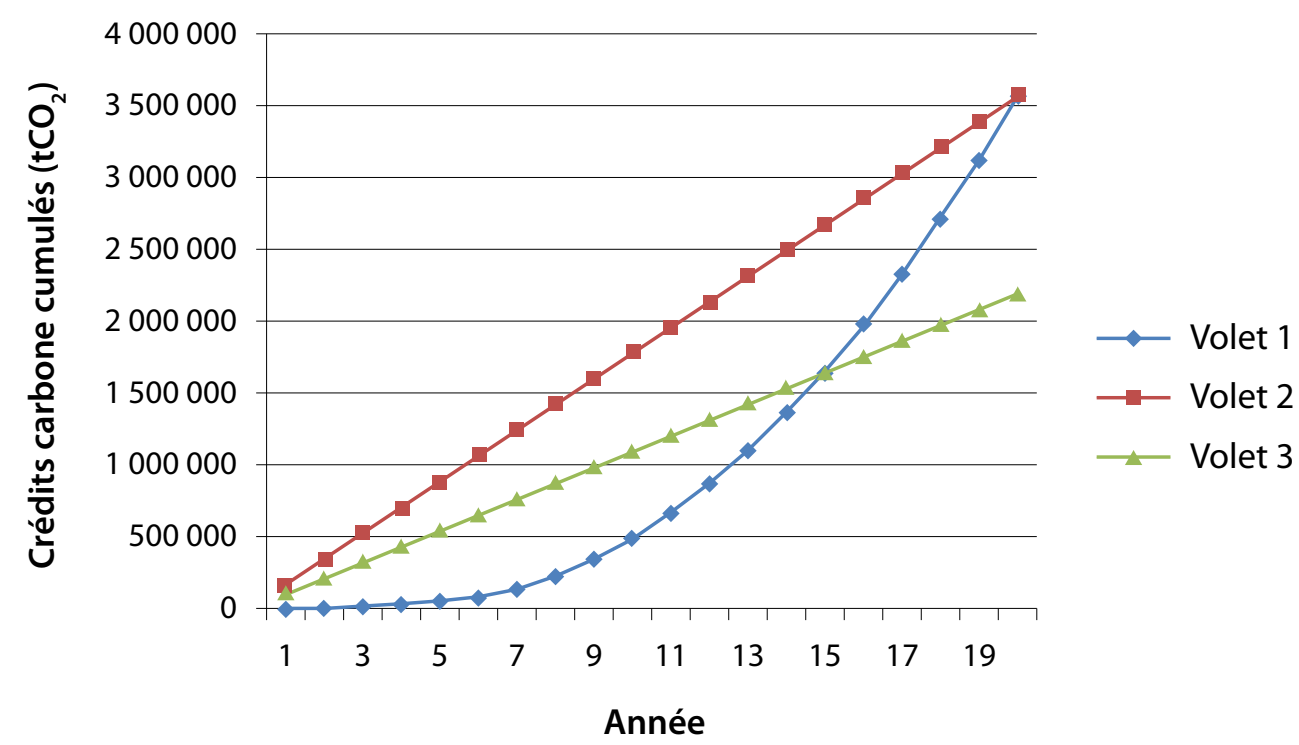

Figure 12 : Crédits carbone cumulés produits par les trois volets Source: FRM 2013

Ce graphique permet de constater que le Volet 1 délivrera peu de crédits carbone les premières années du projet : le Volet 1 ne pourra donc pas être financé par les crédits carbone au démarrage du projet, les évitements d'émissions liés au projet étant en effet en trop faibles quantités par rapport aux financements nécessaires. Par exemple, avec un prix de vente de la tonne de $\mathrm{CO}_{2}$ de 5 euros $/ \mathrm{tCO}_{2} \mathrm{e}$, les investissements du Volet 1 ne seront pas remboursés et le Volet 1 ne deviendra rentable qu'à partir de la dixseptième année (voir Figure 13). Un investissement important de la part de partenaires financiers sera donc indispensable au démarrage, puis, au fur et à mesure de l'avancement du projet, les gains issus de la vente des crédits carbone permettront de financer intégralement les activités du Volet 1 . La combinaison des 3 volets du projet permet de limiter cet important besoin de financement initial, le Volet 1 pouvant être alors en partie financé par la vente des crédits carbone des Volets 2 et 3.

La Figure 14 présente la répartition des montants affectés aux différents postes budgétaires, sur chaque volet individuellement et sur l'ensemble des trois volets (pour un budget total de 30,1 millions d'euros). Ainsi, le budget associé directement au carbone (homologation du projet dans le registre REDD, monitoring) s'élève à $20 \%$ du budget total. C'est la gestion administrative et technique du projet (location de bureaux, personnel d'encadrement et personnel administratif, missions à court terme d'experts internationaux, véhicules) qui représente le poste budgétaire le plus important $(40 \%)$.

Le coût de revient de la tonne de $\mathrm{CO}_{2}$ est modéré pour les trois volets et permettrait donc une insertion du projet sur le marché du carbone en proposant un prix de vente raisonnable. Cependant, il est indéniable que le marché du carbone manque de dynamisme actuellement et que la mise en place d'un projet REDD comporte des risques financiers (risque d'absence de retour sur investissement). Afin de minimiser ces risques, il est conseillé de chercher à valoriser les crédits carbone (ou au moins ceux du Volet 1) sous un standard de grande qualité (CCBS Gold, par exemple) en favorisant les grands bénéfices sociaux et environnementaux du projet.

\subsection{Bénéfices sociaux et environnementaux}

Cela a été démontré plus haut : étant donné le prix relativement élevé du projet (principalement lié à la gestion technique et au personnel d'encadrement, assurant un meilleur taux de succès), le porteur de projet aura tout intérêt à valoriser les crédits carbone sous un standard de haute qualité pour les vendre à un meilleur prix. Pour obtenir ce standard, le projet doit générer des bénéfices sociaux et environnementaux très importants, et c'est indéniablement le cas du projet REDD+ de la Lukénie. 


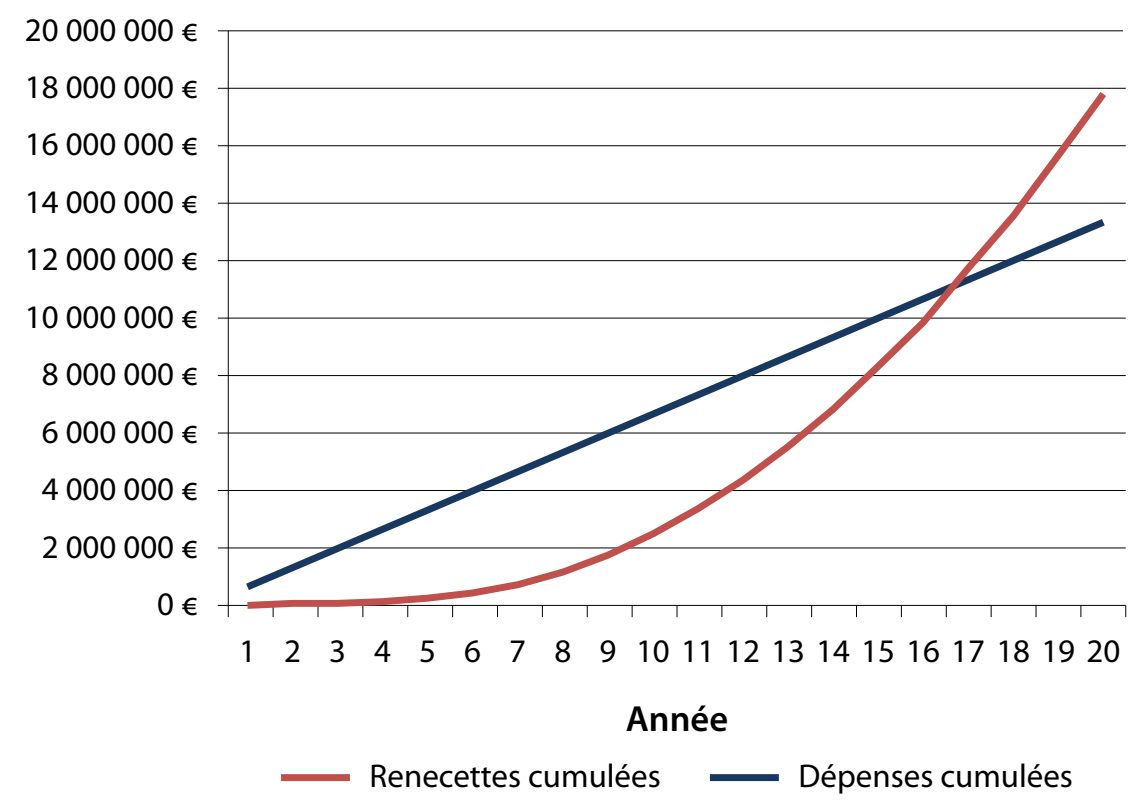

Figure 13 : Recettes et dépenses cumulées du Volet 1 (hypothèse du prix de vente : 5 euros/ $/ \mathrm{CO}_{2}$ )

Source: FRM 2013

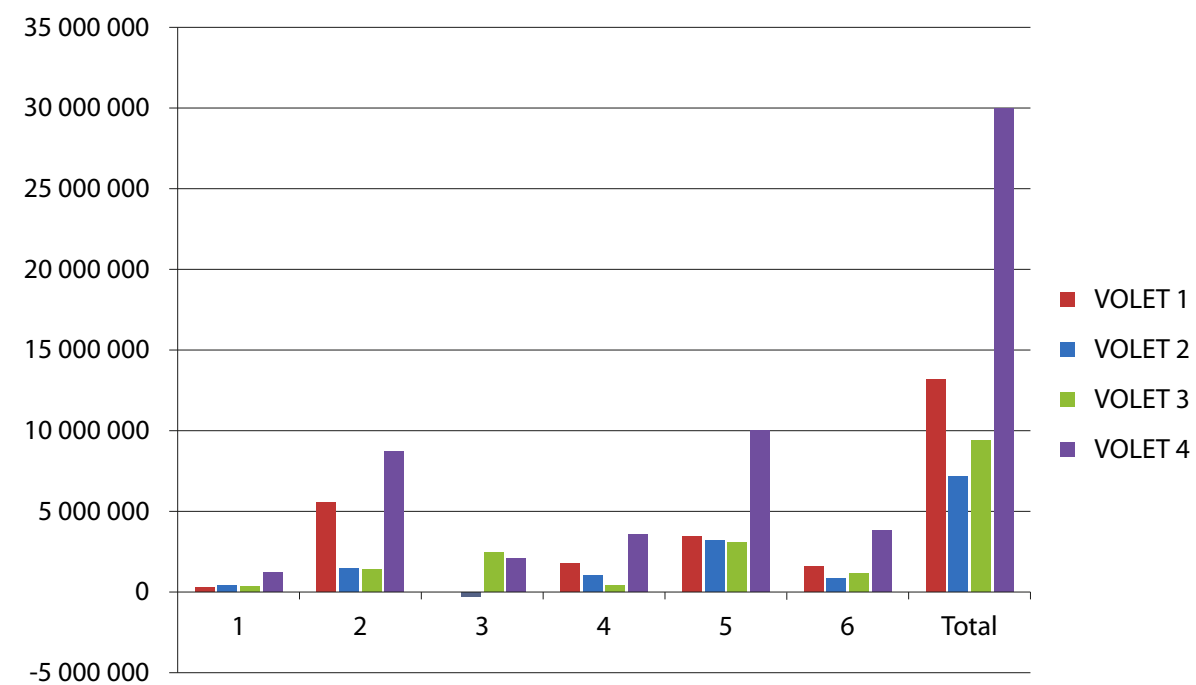

Légende:

\begin{tabular}{|l|l|}
\hline 1 & Phase de préparation du projet REDD \\
\hline 2 & Formation et coût du personnel en charge de la mise en œvre \\
\hline 3 & Coûts liés á la réduction de la productivité ou á l'arrét de la production \\
\hline 4 & Monitoring REDD \\
\hline 5 & Gestion administrative et technique du projet \\
\hline 6 & Imprévus \\
\hline
\end{tabular}

Figure 14 : Ventilation du budget total du projet dans les différentes rubriques Source: FRM 2013 
Tableau 9 : Synthèse des bénéfices environnementaux des trois volets

\begin{tabular}{ll}
\hline Bénéfices environnementaux \\
\hline Sols & Amélioration de la fertilité des sols \\
\cline { 2 - 2 } & Diminution de l'érosion et du lessivage \\
\hline Eau & $\begin{array}{l}\text { Maintien de la qualité des ressources } \\
\text { hydriques }\end{array}$ \\
\hline Biodiversité & $\begin{array}{l}\text { Maintien et amélioration de biodiversité } \\
\text { (faune et flore) grâce à la préservation } \\
\text { des forêts }\end{array}$ \\
\cline { 2 - 2 } & $\begin{array}{l}\text { Maintien des habitats des auxiliaires } \\
\text { de cultures qui limitent l'impact des } \\
\text { parasites sur les productions agricoles }\end{array}$ \\
\cline { 2 - 2 } & $\begin{array}{l}\text { Diminution du dérangement de la faune } \\
\text { lié à l'exploitation forestière }\end{array}$ \\
\hline
\end{tabular}

Les bénéfices environnementaux, comme dans tout projet REDD, découlent principalement de la préservation des forêts naturelles et, par suite, de la préservation de la biodiversité inféodée aux forêts (biodiversité animale et végétale). De plus, ils sont également liés à la préservation des sols (diminution de l'érosion et du lessivage, maintien de la fertilité), grâce aux activités d'agroforesterie.

Les bénéfices sociaux des trois volets sont résumés dans le tableau ci-dessous (Tableau 9).
Le projet entraîne également de nombreux bénéfices socio-économiques, synthétisés ci-dessous (Tableau 10), le Volet 1 " déforestation évitée " étant le plus intéressant de ce point de vue.

\subsection{Risque et gestion du risque}

Tout projet REDD comporte des risques d'échec. Le Volet 1, par exemple, est d'autant plus risqué qu'une multitude d'acteurs est en jeu : l'investissement des populations locales est essentiel pour la réussite $\mathrm{du}$ projet. Avec un seul acteur (la société forestière), le Volet 2 semble en revanche plus maîtrisable : la réussite de ce volet semble moins dépendante du contexte extérieur. Cependant, la méthodologie du Volet 1 existe déjà et n'aura pas besoin d'être modifiée, tandis que celle du Volet 2 reste à développer et celle du Volet 3 à affiner.

Le Tableau 11 présente une synthèse des risques d'échec du projet et les mesures préventives et curatives à prendre en conséquence.

\subsection{L'enregistrement du projet}

Le présent rapport permet de décrire les activités prévues dans le projet ainsi que d'estimer les objectifs de réduction d'émissions. Il s'agit donc des principaux éléments nécessaires à la rédaction du Project design document (PDD), étape préalable à l'enregistrement du projet. Cet enregistrement devra

Tableau 10 : Synthèse des bénéfices sociaux et économiques des trois volets

\begin{tabular}{|c|c|}
\hline \multicolumn{2}{|c|}{ Bénéfices sociaux et économiques } \\
\hline \multirow{6}{*}{$\begin{array}{l}\text { Populations } \\
\text { locales }\end{array}$} & Développement des revenus d'origine agricole \\
\hline & Diversité et sécurité alimentaire \\
\hline & Apprentissage de nouvelles techniques agricoles \\
\hline & $\begin{array}{l}\text { Diminution des défrichements (qui nécessitent des investissements importants en temps et en } \\
\text { énergie) }\end{array}$ \\
\hline & Contribution au développement local (grâce aux bénéfices de la vente des crédits carbone) \\
\hline & $\begin{array}{l}\text { Emploi de personnel local pour la sensibilisation et la mise en œuvre du programme } \\
\text { d'amélioration des techniques agricoles }\end{array}$ \\
\hline \multirow{4}{*}{$\begin{array}{l}\text { Société forestière } \\
\text { (SODEFOR) }\end{array}$} & Maintien du domaine forestier permanent, menacé de déforestation \\
\hline & Amélioration de l'image de la société \\
\hline & Amélioration des relations avec les populations locales \\
\hline & Réduction des charges associées à l'ouverture des routes \\
\hline \multirow{4}{*}{$\begin{array}{l}\text { État (République } \\
\text { démocratique } \\
\text { du Congo) }\end{array}$} & Développement de projet pilotes rentrant dans la stratégie nationale REDD+ \\
\hline & Maintien du domaine forestier permanent, menacé de déforestation \\
\hline & Diminution de la pauvreté \\
\hline & Reproductibilité du projet (attraction d'investisseurs, etc.) \\
\hline
\end{tabular}


Tableau 11 : Risques et gestion du risque

\begin{tabular}{|c|c|c|}
\hline $\begin{array}{l}\text { Volet } \\
\text { REDD+ }\end{array}$ & Risque identifié & Préconisations en conséquence \\
\hline \multirow[t]{3}{*}{ Volet 1} & Non adhésion des populations locales & $\begin{array}{l}\text { Présence de plusieurs animateurs agricoles répartis sur } \\
\text { les différents villages des concessions ; sensibilisation et } \\
\text { démonstrations pilotes bien répartis sur le territoire d'étude. }\end{array}$ \\
\hline & $\begin{array}{l}\text { Dépassement des limites prévues pour } \\
\text { les défrichements }\end{array}$ & $\begin{array}{l}\text { Bornage des zones affectées au développement rural et } \\
\text { sensibilisation des populations locales. Reconnaissance du } \\
\text { droit coutumier en droit moderne. }\end{array}$ \\
\hline & $\begin{array}{l}\text { Risque de destruction des parcelles } \\
\text { améliorées par des feux non contrôlés }\end{array}$ & $\begin{array}{l}\text { Réflexion à considérer lors de la mise en place des parcelles } \\
\text { améliorées. Maintien d'une ceinture pare-feu ou d'une } \\
\text { végétation verte en contour. }\end{array}$ \\
\hline \multirow[t]{2}{*}{ Volet 2} & Risque d'échec des mesures forestières & $\begin{array}{l}\text { Suivi rigoureux de l'évolution des stocks de carbone, } \\
\text { adaptation des activités en conséquence. }\end{array}$ \\
\hline & Absence de méthodologie & $\begin{array}{l}\text { Elaboration et validation de la méthodologie avant } \\
\text { enregistrement du projet. }\end{array}$ \\
\hline Volet 3 & Activités d'exploitation illégales & $\begin{array}{l}\text { Renforcement des contrôles, sensibilisation, agents de } \\
\text { surveillance. }\end{array}$ \\
\hline Tous & $\begin{array}{l}\text { Absence de résultats et manque de } \\
\text { revenus carbone }\end{array}$ & $\begin{array}{l}\text { Scénario de référence conservatif et scénario de } \\
\text { projet prudent. }\end{array}$ \\
\hline
\end{tabular}

se faire en suivant une procédure d'homologation nationale obligatoire pour tous les projets REDD+ de République démocratique du Congo. Un manuel de procédure pour l'homologation nationale a été publié en janvier 2012 par le Ministère de l'environnement, conservation de la nature et tourisme (MECNT) pour guider les porteurs de projet, et a été fixé par l'arrêté ministériel ${ }^{\circ}$ 004/CAB/MIN/ECN-T/012 du 15 février 2012.

Avant de chercher à enregistrer le projet, il faudra mettre en place les partenariats indispensables au bon déroulement du projet. Le Volet 1, en particulier, exigera la présence d'une ONG et/ou d'une structure spécialisée dans le développement agricole et l'agroforesterie (université, société spécialisée dans le développement rural, etc.). Cette structure devra également être en mesure de fournir aux populations les plants et les boutures nécessaires (notamment des boutures de manioc résistant à la mosaïque). La mise en ouvre des Volets 2 et 3 pourra être appuyée par FRM, un bureau d'études spécialisé en foresterie.

Outre ces partenariats techniques, il faudra également mettre en place des partenariats financiers : comme le montrent les budgets des trois volets, chaque volet aura besoin d'investissements importants au départ. Certes, le projet bénéficiera d'un retour sur ces investissements lors de la vente des crédits carbone, mais les investissements initiaux n'en nécessiteront pas moins une trésorerie importante pour amorcer la mise en œuvre des trois volets. Avant d'enregistrer le projet, il faudra donc rechercher des partenaires financiers et s'assurer un accord pour la vente des crédits carbone avec un acheteur de carbone forestier.

Avant la phase d'enregistrement, le contexte local et institutionnel devra également être étudié afin de déterminer les éventuelles options en cohérence avec les projets en cours. Ainsi, le projet devra chercher à s'intégrer dans la stratégie provinciale REDD du Bandundu, actuellement en cours d'élaboration. Audelà de la simple inscription au registre des projets REDD en cours dans la province, des synergies pourront être recherchées. En effet, cette stratégie provinciale ${ }^{14}$ prévoit notamment un programme de sensibilisation et d'éducation environnementale auprès des populations en diffusant les techniques d'agroforesterie et d'agriculture écologiquement intensive sans recourir au brûlis. Un renforcement réciproque des capacités sera donc possible si ce programme provincial et le Volet 1 du projet pilote $\mathrm{REDD}+$ sont conçus en partenariat.

Par ailleurs, le plus grand projet REDD+ jamais validé à ce jour a effectué sa première livraison de crédits carbone le $1^{\text {er }}$ février 2013. Il s'agit du premier

14 En cours d'élaboration. Contacter Jean-Jacques Bambuta, au point focal REDD+ du Bandundu. 


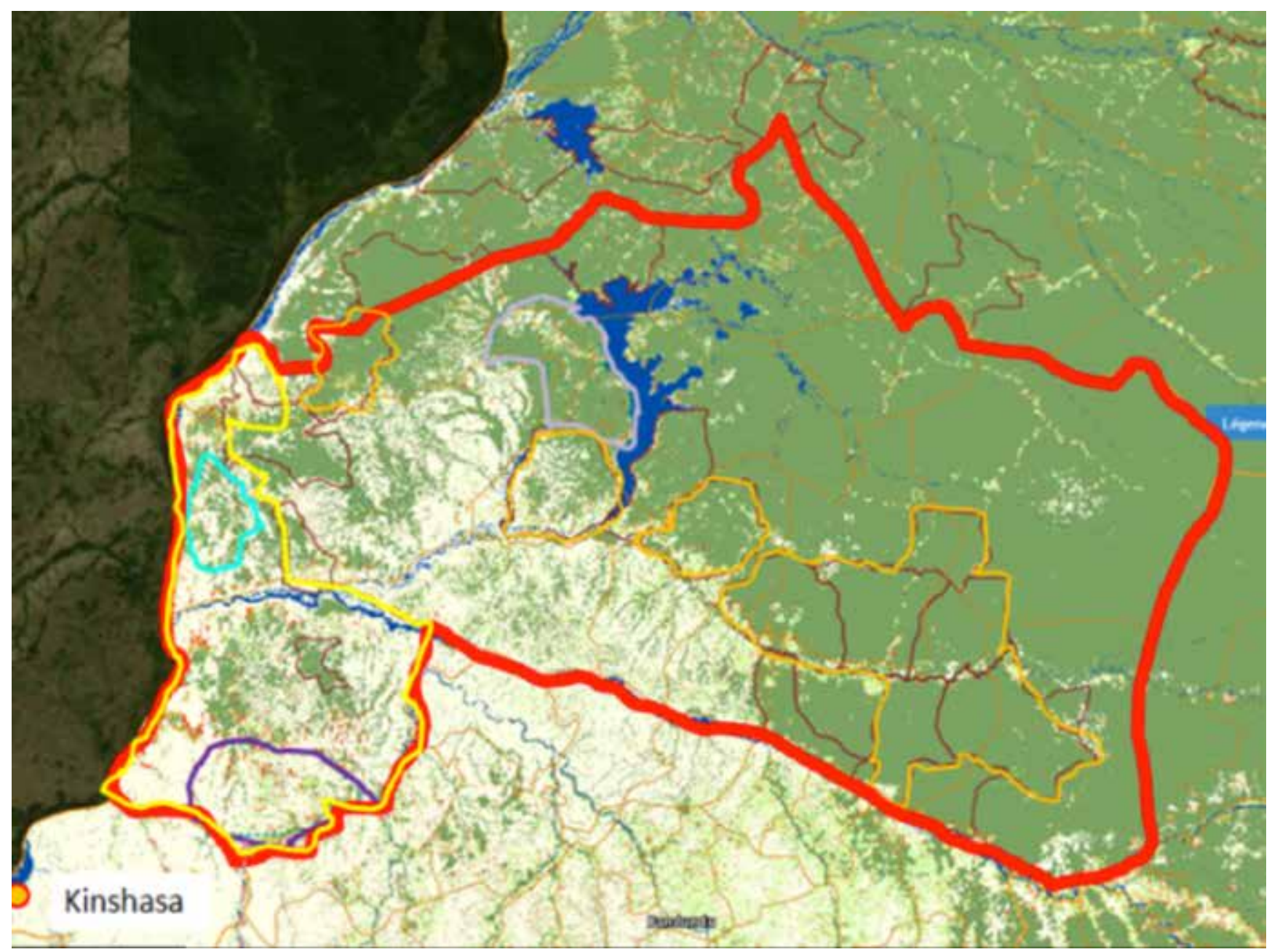

Figure 15 : Initiatives REDD+ en cours dans le district du Maï Ndombé

Source: Coordination nationale REDD+ de la République Démocratique du Congo

projet REDD du bassin du Congo à avoir été validé et vérifié, et ce projet pionnier concerne justement la province du Bandundu, en République démocratique du Congo, à une centaine de kilomètres à l'ouest du projet pilote REDD+ de la Lukénie. Ce projet "Maï Ndombe REDD+ " ${ }^{15}$ est constitué de plusieurs activités :

- L'interruption de l'exploitation forestière légale (mise en conservation volontaire) ;

- La réduction de l'exploitation illégale ;

- L'amélioration des techniques agricoles ;

- Le développement d'infrastructures et d'activités socio-économiques.

Là encore, des bénéfices réciproques pourraient être réalisés si les deux porteurs de projet travaillent ensemble en échangeant leurs expériences.

15 The Maï Ndombe REDD+ Project, a joint project of ERA and Willdlife Works, version 1.63 du 19 novembre 2012
De manière plus générale, différents projets REDD+ sont en cours d'élaboration dans la région. L'ONU$\mathrm{REDD}$ en $\mathrm{RDC}$ a donc proposé de rassembler toutes ces initiatives au sein d'un grand projet REDD+ qui couvrirait à terme tout le district du Maï Ndombe (voir Figure 15), afin d'améliorer la visibilité du projet et de mutualiser les efforts de vente des crédits carbone. Le porteur du projet pilote REDD+ de la Lukénie pourra ainsi faire le choix de s'insérer dans ce grand projet.

La plupart des éléments nécessaires à l'élaboration du PDD sont fournis dans le présent rapport. Certains points restent néanmoins à approfondir.

Notamment, le sujet de l'additionnalité n'a pas été abordé ici. Celle-ci ne devrait pas être difficile à démontrer, particulièrement en ce qui concerne les Volets 1 et 3, puisque le coût des opérations et la perte de bénéfice (interruption de l'exploitation) prouvent directement que ces projets ne seraient pas économiquement viables sans l'apport des 
crédits carbone. L'additionnalité du Volet 2 devra quant à elle être examinée avec plus d'attention : la substitution des routes par des pistes de débardage permettra de faire des économies importantes sur la longueur de routes à construire. Mais les activités du Volet 2 auront également d'autres répercussions - plus indirectes - sur l'exploitation, comme l'augmentation du nombre de jours nécessaires au transport du bois (à cause de la réduction des bandes d'ensoleillement et, donc, du nombre de jours où la route est sèche et carrossable). Si cette incidence particulière a été modélisée, il existe probablement d'autres conséquences indirectes qui devront être examinées avant le lancement des activités pour aider le concessionnaire à prévoir au mieux les modifications de son exploitation et les répercussions financières. Ces éléments permettront également de mieux préciser les barrières financières qui expliqueraient l'additionnalité du projet.

Par ailleurs, les fuites (leakages) demanderont également à être précisées lors de l'élaboration du PDD. Les populations des concessions 28/03 et 30/03 risquent-elles par exemple de déplacer leurs activités agricoles en dehors des concessions du projet, entraînant ainsi des émissions de $\mathrm{CO}_{2}$ en dehors de la zone d'étude à cause des activités REDD ? Cette question concerne essentiellement les villages situés en bordure de concession et devra faire l'objet d'une étude approfondie pour estimer ce risque. De même, l'arrêt de l'exploitation dans les concessions 24/03 et 29/03 risque-t-il d'entraîner une surexploitation dans les concessions voisines? Cela est peu probable, car les plans d'aménagement des autres concessions sont déjà en grande partie élaborés, mais une étude plus approfondie sera peutêtre nécessaire.

Enfin, la mise en place d'un "buffer" (fonds de réserve) sera nécessaire pour limiter le risque d'insuffisance de résultats. Ainsi, un buffer de $30 \%$ sur les crédits carbone pourrait être appliqué ${ }^{16}$. Cet élément devra être renseigné dans le PDD.
16 Assurance de crédits carbone recommandée par le VCS. Voir VCS Tool for AFOLU Non-Permanence Risk Analysis and Buffer. 


\section{Conclusion}

Le projet pilote REDD+ de la Lukénie vise à réduire la déforestation et à améliorer les stocks de carbone forestier en jouant essentiellement sur trois leviers :

- La limitation des défrichements liés à l'agriculture vivrière, en mettant en place une agriculture écologiquement intensive moins consommatrice de superficies forestières ;

- La diminution des conséquences de l'exploitation sur les stocks de carbone forestier, en mettant en place une exploitation forestière à impact réduit et en minimisant la longueur des routes secondaires ;

- L'arrêt de l'exploitation forestière dans certaines concessions, par mise en conservation volontaire.

L'objectif principal de l'analyse faite dans le présent rapport est d'étudier la faisabilité et la reproductibilité d'un projet REDD+ au sein d'une concession forestière en République démocratique du Congo, et d'évaluer l'incidence de différentes activités REDD+ sur les stocks de carbone forestier.

Après étude, il apparaît que ce projet présente un intérêt notable pour toutes les parties prenantes : des bénéfices environnementaux et sociaux incontestables pour les écosystèmes et les populations locales, ainsi que des bénéfices en termes d'image pour la société forestière porteuse du projet. De plus, ces activités REDD+ sont susceptibles d'éviter d'importantes émissions de $\mathrm{CO}_{2}$, donc d'engendrer des quantités importantes de crédits carbone : finalement, ce projet génère donc également des bénéfices financiers très importants pour les populations locales, la société forestière et l'État. Outre ces bénéfices sociaux, écologiques et économiques directs, les bénéfices indirects liés à la préservation de la forêt et de la biodiversité, plus difficiles à estimer, ne peuvent être négligés.

Les atouts d'un tel projet sont donc indéniables s'il est mené à terme. Mais les risques d'échec ne sont pas négligeables : risque de non-adhésion des populations locales, risque d'inefficacité des mesures préconisées, risque d'instabilité politique, etc. Ce projet devra donc être suivi avec rigueur et attention afin de s'adapter sans cesse au contexte changeant et de proposer de nouvelles options et des améliorations en fonction des problèmes rencontrés au cours de l'avancement du projet. Cependant, une part du risque ne pourra pas être occultée et le porteur de projet doit en être conscient.

Enfin, les différents éléments nécessaires à l'établissement du PDD et à l'enregistrement du projet ont été rassemblés et calculés pour l'élaboration du présent rapport. Seuls quelques points restent à clarifier, notamment la justification de l'additionnalité, l'estimation des fuites et la définition du buffer. D’autres points nécessiteront une étude plus approfondie également, comme la définition de la zone de référence pour le Volet 1 (et l'estimation du scénario de référence sur cette zone), ainsi que le développement et la validation d'une méthodologie novatrice pour les Volets 2 et 3, en s'appuyant sur ce qui a été proposé dans le présent rapport.

Ensuite, le projet pourra faire l'objet d'une homologation et être inscrit au registre des projets REDD+ : une vérification des émissions réellement évitées sera effectuée tous les trois ans et les crédits carbone seront délivrés en fonction des résultats, puis redistribués entre les différentes parties prenantes selon des modalités qui auront été fixées au préalable.

La collaboration avec des bailleurs de fonds s'avérera probablement nécessaire pour amorcer la mise en œuvre du projet. Surtout, des partenariats devront vraisemblablement être établis avec des ONG et différentes institutions, telles que le point focal REDD du Bandundu, le comité national REDD de RDC et le comité international du VCS : des synergies pourront être trouvées afin de faciliter la mise en œuvre de la stratégie REDD sur le terrain en République démocratique du Congo.

Ce projet, bien qu'ambitieux et complexe, est tout à fait réalisable et les nombreux bénéfices économiques, sociaux et écologiques lui donnent tout son intérêt. Il s'agit d'un projet reproductible en République démocratique du Congo si les mêmes pressions sont observées sur la forêt et s'il emporte l'adhésion d'une compagnie forestière et des populations locales; s'il n'est pas reproduit intégralement, l'un des volets du 
projet peut au moins être choisi individuellement pour faire l'objet d'un projet REDD+ puisque les trois volets sont indépendants.

Toutefois, le projet REDD+ de la Lukénie tire incontestablement sa spécificité des trois volets qui le composent : trois volets certes indépendants mais également complémentaires. Ainsi, comme cela a été démontré plus haut, le Volet 1 pourra être au moins partiellement financé par les deux autres volets au lancement du projet. Le Volet 3 est celui qui comporte le moins de risques d'échecs (puisqu'il dépend quasi uniquement de la volonté du porteur de projet), mais le Volet 1 est celui qui apporte le plus de bénéfices socio-économiques et qui, par suite, contribue à améliorer l'image de la société forestière. Le Volet 2 quant à lui semble le plus rentable financièrement. C'est donc l'association de ces trois volets qui donne toute sa force au projet REDD+ de la Lukénie.

Dans tous les cas, la réussite de ce projet dépendra du dynamisme du marché du carbone mondial. Très peu de projets REDD+ ont été enregistrés dans le monde et un seul en Afrique, les crédits carbone délivrés par le projet étant actuellement difficiles à vendre. Le succès intégral du projet dépend donc grandement du contexte externe, et nécessite notamment des politiques mondiales fortes dans le domaine $\mathrm{du}$ marché du carbone.

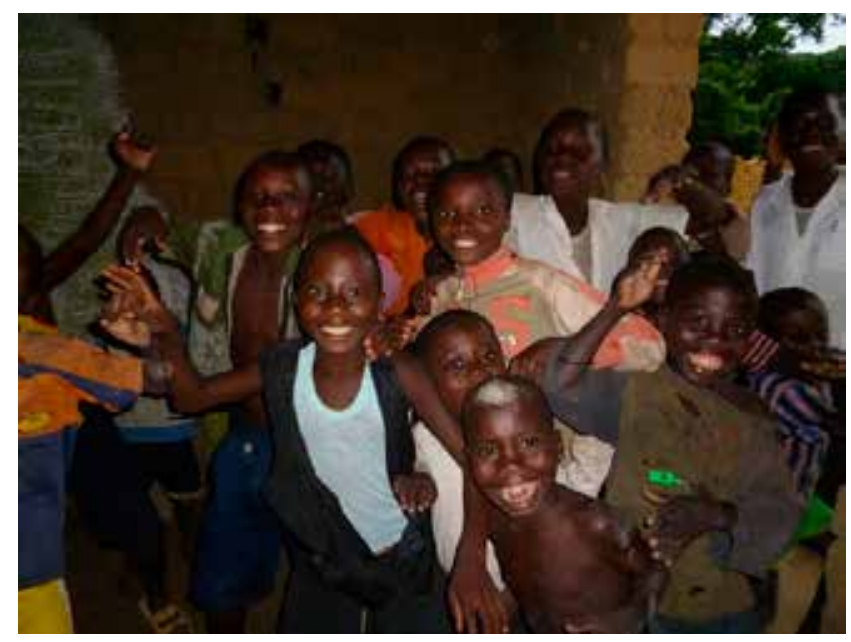

Groupe d'enfants du village d'Ekwayolo Source: FRM 2013 

Les documents de travail du CIFOR contiennent des résultats préliminaires ou anticipés concernant d'importantes questions sur les forêts tropicales qui nécessitent d'être publiées à l'avance.

Ces documents sont produits pour informer et inciter la discussion. Leur contenu a été revisé à l'interne, mais n'a pas subi la procédure d'examen par les pairs.

Dans le cadre du projet FORAFAMA, FRM a étudié les potentialités d'un projet REDD+ en République Démocratique du Congo, dans les concessions forestières de la Lukénie (province du Bandundu). Le présent rapport est constitué des travaux analytiques préalables à la mise en œuvre de ce projet REDD+ : estimation des stocks de carbone de l'état initial (scénario de référence), développements méthodologiques, calcul des changements de stocks, mise en place de protocoles de suivi, éléments d'analyse financière.

Ce projet s'articule autour de trois activités :

- Volet 1 - Déforestation évitée : réduire la dynamique de déforestation liée à l'agriculture vivrière et stabiliser progressivement l'agriculture sur les surfaces déjà défrichées ;

- Volet 2 - Exploitation Forestière à Impact Réduit (EFIR) : réduire les émissions de carbone liées au prélèvement de bois d'œuvre par la SODEFOR ;

- Volet 3 - Conversion d'une forêt exploitée en réserve intégrale : mettre en conservation volontaire (interrompre l'exploitation) une partie des concessions.

Les principales activités du volet 1 concernent la diffusion de nouvelles pratiques agricoles, notamment agroforestières, afin de pallier les problèmes de fertilité et d'érosion des sols. Ces activités devraient permettre de diminuer la pression sur les forêts naturelles, avec un objectif de près de 8000 ha de déforestation évitée en 20 ans, soit 3,5 millions de crédits carbone.

Le volet 2 prévoit une meilleure planification des réseaux d'évacuation des bois, avec la substitution des routes secondaires par des pistes de débardage plus longues (celles-ci ayant un impact moins important sur les stocks de carbone forestier) : en 20 ans, près de 3,6 millions de crédits carbone devraient ainsi être générés.

Dans le volet 3, c'est l'impact de la mise en conservation volontaire qui a été étudiée (aucun prélèvement d'arbre ni création de réseau routier) : près de 2,2 millions de crédits carbone devraient ainsi être générés.

\begin{tabular}{l|l} 
Cette recherche a été menée par le CIFOR dans le cadre du Programme de recherche du CGIAR \\
sur les forêts, les arbres et l'agroforesterie (CRP-FTA). Ce programme collaboratif vise à améliorer la \\
gestion et l'utilisation des forêts, de l'agroforesterie et des ressources génétiques des arbres à l'échelle \\
RECHERGE SUR \\
les Forêts, les Arbres et
\end{tabular}
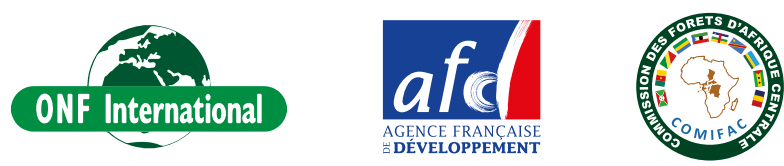

Centre de recherche forestière internationale (CIFOR)

Le CIFOR oeuvre en faveur du bien-être humain, de la conservation de l'environnement et de l'équité par sa recherche scientifique qui contribue à l'élaboration des politiques et des pratiques affectant les forêts dans les pays en développement. Le CIFOR est membre du Consortium du CGIAR. Son siège est situé à Bogor en Indonésie et il est également implanté en Asie, en Afrique et en Amérique du Sud. 